\title{
STATISTICAL MECHANICS OF INTERPOLATION NODES, PLURIPOTENTIAL THEORY AND COMPLEX GEOMETRY
}

\author{
ROBERT J. BERMAN
}

\begin{abstract}
This is mainly a survey, explaining how the probabilistic (statistical mechanical) construction of Kähler-Einstein metrics on compact complex manifolds, introduced in a series of works by the author, naturally arises from classical approximation and interpolation problems in $\mathbb{C}^{n}$. A fair amount of background material is included. Along the way, the results are generalized to the non-compact setting of $\mathbb{C}^{n}$. This yields a probabilistic construction of Kähler solutions to Einstein's equations in $\mathbb{C}^{n}$, with cosmological constant $-\beta$, from a gas of interpolation nodes in equilibrium at positive inverse temperature $\beta$. In the infinite temperature limit, $\beta \rightarrow 0$, solutions to the Calabi-Yau equation are obtained. In the opposite zero-temperature the results may be interpreted as "transcendental" analogs of classical asymptotics for orthogonal polynomials, with the inverse temperature $\beta$ playing the role of the degree of a polynomial.
\end{abstract}

\section{INTRODUCTION}

The main purpose of the present work is to explain how the probabilistic (statistical mechanical) construction of Kähler-Einstein metrics on compact complex manifolds - which is the subject of the series of works [19, 18, 15, 21 - naturally arises from classical approximation and interpolation problems in $\mathbb{C}^{n}$. In particular, the connection to Siciak's conjecture [104 (proved in [27]) about the convergence of Fekete points towards the pluripotential equilibrium measure is stressed.

In a nutshell, Fekete points may be defined as optimal interpolation nodes. As will be explained below, from the statistical mechanical point of view they are minimal energy configurations, which arise at zero-temperature. When the temperature is increased (twisted) Kähler-Einstein metrics emerge. See [14] for different motivations coming from physics, in particular emergent gravity and quantum gravity and [19] for motivations coming from algebraic geometry. We also provide sufficent background from both complex geometry and probability in order to explain the proofs of the main results and to put the results into a general context. This work is thus mainly expository. But compared to the original papers more emphasize will be put on the notion of Gamma-convergence.

We also take the opportunity to show how the results in the compact setting can be used to obtain new results in the non-compact setting of $\mathbb{C}^{n}$. This leads, among other things, to "transcendental" analogs of classical asymptotics for orthogonal polynomial, with the inverse temperature $\beta$ playing the role of the degree of a polynomial. The paper is concluded with an outlook section where open problems are discussed.

The main results may be summarized as follows in the non-compact setting of $\mathbb{C}^{n}$. Assume given a smooth function $\phi$ in $\mathbb{C}^{n}$ with sufficent growth at infinity (super logarithmic) and a

Key words and phrases. pluricomplex Green functions, complex Monge-Ampère operators, point processes, large deviations. MSC 2010 subject classifications: 32U35, 32W20, 60G55, 60F10 . 
positive number $\beta$. We will introduce a probabilistic construction of solutions $\omega_{\beta}$ to Einstein's equation

$$
\operatorname{Ric} \omega+\beta \omega=\tau_{\beta, \phi}
$$

for a Kähler metric $\omega$ on $\mathbb{C}^{n}$, where $\tau_{\beta, \phi}$ is the symmetric tensor

$$
\tau_{\beta, \phi}=\beta \omega^{\phi}
$$

with $\omega^{\phi}$ denoting the complex Hessian of the given function $\phi$. The solution $\omega_{\beta}$ will be constructed by an explicit sampling procedure involving "random interpolation nodes" in $\mathbb{C}^{n}$ with $\phi$ plaiyng the role of a "weight function". From a statistical mechanical point of view the role of the interpolation nodes is played by interacting particles in thermal equilibrium. The parameter $\beta$ corresponds to the inverse temperature and $\phi$ to an exterior confining potential. This can be seen as a higher-dimensional generalization of the classical principle that interpolation nodes in $\mathbb{C}$ tend to behave as repulsive electric charges. However, in the present higher dimensional setting the corresponding physical model is provided by the theory of general relativity, rather than electrostatics. Indeed, the metric $\omega_{\beta}$ is a solution to Einstein's equation on $\mathbb{C}^{n}$ (with Euclidean signature) with cosmological constant $-\beta$ and prescribed (trace-reversed) stress-energy tensor $T_{\beta, \phi}$ (the solution $\omega_{\beta}$ is uniquely determined by condition that its Kähler potential $\psi_{\beta}$ has logarithmic growth at infinity). From the complex geometric point of view such metrics are twisted Kähler-Einstein metrics, which play an important role in current complex geometry in connection to the Yau-Tian-Donaldson conjecture, where the role of $\mathbb{C}^{n}$ is played by a compact complex manifold (see the survey [54]).

We also show that in the "zero temperature limit" $(\beta \rightarrow \infty)$ the Kähler potential $\psi_{\beta}$ of $\omega_{\beta}$ converges towards Siciaks weighted extremal function, which is the potential of the weighted pluripotential equilibrium measure. In the opposite "infinite temperature limit" $(\beta \rightarrow 0)$ we instead recover a solution to the Calabi-Yau equation in $\mathbb{C}^{n}$. Finally, the tropicalization of the negative temperature regime is discussed, where Kähler-Einstein metrics on toric varieties arise as $\beta$ approaches a certain critical negative inverse temperature. Relations to Optimal Transport theory are also briefly mentioned.

1.1. Organization. We start in Section 2 by giving a bird's-eye view, providing motivation from interpolation theory and stating the main results from [27, 18]. The new results are given in Theorems 2.7. Theorem 2.12 and Theorem 2.15. In the following two sections we zoom in on the details, providing background from complex geometry and probability. The proofs of the main results are then explained in Sections 5, 6. Finally, in Section 7 an outlook on open problems is provided. In the appendix the present setting is compared with the classical Curie-Weiss model for magnetization in spin systems.

1.2. Acknowledgements. Thanks to Slawomir Kolodziej for the invitation to contribute to the upcoming volume in Annales Polonici Mathematici, in memory of Jozef Siciak, which prompted the present work. It is also a pleasure to thank Sebastien Boucksom, David WittNyström, Vincent Guedj and Ahmed Zeriahi for the stimulating collaborations [26, 27, 28], which paved the way for the probabilistic approach and Mingchen Xia for comments on a draft of the present manuscript. This work was supported by grants from the ERC and the KAW foundation. 


\section{A BIRD'S-EYE VIEW}

2.1. The classical one-dimensional setting. A central problem in approximation theory is to find good points ("nodes") for interpolating polynomials of large degree, on a given compact subset $K$ in $\mathbb{C}^{n}$. We start by recalling the classical one-dimensional case of this problem, which has been studied extensively both from a theoretical, as well as a numerical point of view with classical contributions from Gauss, Fekete, Fejer, Szegö, Chebishev and many others. We thus assume given a compact subset $K$ of $\mathbb{C}$. Denote by $\mathcal{P}_{k}(\mathbb{C})$ the complex vector space of all polynomials on $\mathbb{C}$ of degree at most $k$. This space has dimension

$$
N_{k}:=\operatorname{dim} \mathcal{P}_{k}(\mathbb{C})=k+1
$$

A given configuration $\left(z_{1}, \ldots z_{N_{k}}\right)$ of $N_{k}$ points on $K$ determines an evaluation map

$$
\operatorname{ev}_{\left(z_{1}, \ldots, z_{N_{k}}\right)}: \mathcal{P}_{k}(\mathbb{C}) \rightarrow \mathbb{C}^{N_{k}}, \quad p_{k} \mapsto\left(p_{k}\left(z_{1}\right), \ldots, p_{k}\left(z_{N_{k}}\right)\right)
$$

The worst case scenario is when the determinant of this map vanishes. Indeed, then the evaluation map cannot be inverted, which means that there are values in $\mathbb{C}^{N_{k}}$, which cannot be interpolated by any polynomial $p_{k}$ of degree at most $k$. But even if the determinant does not vanish a generic configuration of points typically leads to unstable interpolation for large degree polynomial. This is illustrated by the case when $K$ is an interval in $\mathbb{R} \subset \mathbb{C}$ and the points are equidistant. Interpolation then results in polynomials that oscillate wildly between the interpolation points, especially close to the boundary (Runge's phenomenen). Accordingly, a natural candidate for good interpolation points are configurations of points $\left(z_{1}, \ldots z_{N_{k}}\right)$ on $K$ maximizing the absolute value of the determinant of the evaluation map 2.1 Such points are called Fekete points for $K$. Picking a base $e_{1}, \ldots e_{N_{k}}$ in the vector space $\mathcal{P}_{k}(\mathbb{C})$ the determinant of the evaluation map 2.1 becomes

$$
D\left(z_{1}, \ldots, z_{N_{k}}\right):=\operatorname{det}\left(e_{i}\left(z_{j}\right)\right)_{i, j \leq N_{k}},
$$

which is called the Vandermonde determinant in the standard case when $e_{i}(z):=z^{i}$ is taken as the monomial base. Since the set $K$ is assumed compact it always admits Fekete points, for any given degree $k$. Finding explicit Fekete points is, in general, an intractable problem which has only been solved for very particular examples of $K$ (se below). But their asymptotic distribution as $k \rightarrow \infty$ is, in general, described by the following classical result (see [98] for a proof):

Theorem 2.1. Assume that $K$ is compact subset of $\mathbb{C}$ which is non-polar. Let $\left(z_{1}, \ldots z_{N_{k}}\right) \in$ $K^{N}$ be a sequence of Fekete points on $K$. Then

$$
\frac{1}{N_{k}} \sum_{i=1}^{N_{k}} \delta_{z_{i}} \rightarrow \mu_{K}
$$

in the weak topology of measures on $K$, where $\mu_{K}$ is the potential theoretic equilibrium measure of $K$. In other words, $K$ is the unique minimizer of the logarithmic energy, i.e. of the following functional, defined on the space $\mathcal{M}_{1}(K)$ of all probability measures on $K$ :

$$
E(\mu)=-\frac{1}{2} \int_{\mathbb{C} \times \mathbb{C}} \log |z-w|^{2} \mu(z) \otimes \mu(w)
$$


The starting point of the proof is the observation that $D\left(z_{1}, \ldots, z_{N_{k}}\right)$ may be factorized as

$$
D\left(z_{1}, \ldots, z_{N_{k}}\right)=\prod_{i<j}\left(z_{i}-z_{j}\right)
$$

This follows directly from the fact that the determinant $D\left(z_{1}, \ldots, z_{N_{k}}\right)$ vanishes if two points coincide and restricts to a polynomial of degree $k$ on $\mathbb{C}$ when all but one arguments are frozen. Replacing the maximizing problem for $|D|$ with the equivalent minimization problem for $-\log |D|^{2}$ thus reveals that the Fekete points on $K$ are precisely the minimiers of the discrete (normalized) logarithmic energy

$$
E^{(N)}\left(z_{1}, \ldots z_{N}\right):=-\frac{1}{N(N-1)} \log |D|^{2}=-\frac{1}{N(N-1)} \frac{1}{2} \sum_{i \neq j} g\left(z_{i}, w_{j}\right),
$$

with a repulsive logarithmic pair interaction potential:

$$
g(z, w):=-\log |z-w|^{2}
$$

In physical terms this is the (normalized) interaction energy betweem $N$ identical particles interacting by the pair interaction potential $g(z, w)$, defined by Green function of the Laplacian on $\mathbb{C}$ (with the divergent self-interactions removed). The minimizers thus represent $N$ equally charged particles confined to $K$ and in electrostatic equilibrium. If the pair interaction $g$ were continuous it would follow that the corresponding energies are related by

$$
E_{g}^{(N)}\left(z_{1}, \ldots z_{N}\right)=E_{g}\left(\frac{1}{N} \sum_{i=1}^{N} \delta_{z_{i}}\right)+o(1)
$$

as $N \rightarrow \infty$, which implies Theorem 2.1 in the continuous setting. Even if the previous relation drastically fails when $g$ is logarithmic (since the right hand side is alway divergent) a truncation argument can be used to essentially reduce the proof of Theorem 2.1 to the case when $g$ is continuous.

Example 2.2. When $K$ is a circle the Fekete points are precisely the configurations consisting of equidistant points and their large $k$-limit $\mu_{K}$ is thus the unique invariant probality measure on the circle. In this case the space $\mathcal{P}_{k}(\mathbb{C})$ identifies with Fourier series with "band-limited" frequences is $[0, k]$, For $K=[-1,1] \Subset \mathbb{R} \subset \mathbb{C}$ the Fekete points are precisely the GaussLobatoo points (which may be realized as the zeroes of Jacobi polynomials) and $\mu_{K}$ is the arcsine distribution $\pi^{-1}\left(1-|x|^{2}\right)^{-1 / 2} d x[66]$.

The case when $K$ is a circle illustrates a striking phenomen: even if $E^{(N)}$ has, in general, a multitude of minimizers in $K^{N}$ the functional $E(\mu)$ always has a unique minimizer. The equidistribution result in Theorem 2.1 applies more generally to asymptotic Fekete points on $K$, i.e. configuration of points on $X$ such that $E^{\left(N_{k}\right)}\left(z_{1}, \ldots z_{N_{k}}\right)$ is equal to the minimum value of $E^{\left(N_{k}\right)}$, up to an error tending to zero as $k \rightarrow \infty$. This rather flexible notion enpasses many classical configuration of points such as Lesbegue points (aka optimal interpolation nodes, as they minimize the $L^{\infty}$ - operator norm of the evaluation map) and zeroes of degree $k$ polynomials $p_{k}$ which are orthogonal with respect to a measure with support $K$ (e.g. zeros of Chebishev polynomials when $K=[-1.1])$.

From a physical point of view the upshot of Theorem 2.1 and its proof is that nearly optimal interpolation behave as repulsive charged particles in (or close to) electrostatic equilibrium. 
2.1.1. The equilibrium potential as an envelope. Before turning to the higher dimensional setting we recall some alternative expressions for the logarithmic energy $E(\mu)$ and its minimizer $\mu_{K}$. First, if follows directly from the defininition that

$$
E(\mu)=-\frac{1}{2} \int_{\mathbb{C}} \psi_{\mu} \mu, \psi_{\mu}(z):=\int_{\mathbb{C}} \log |z-w|^{2} \mu(w)
$$

where $\psi_{\mu}$ is a potential for the measure $\mu$, i.e. $\psi_{\mu}$ is as a solution of the (normalized) Laplace equation in $\mathbb{C}$ :

$$
\frac{1}{4 \pi} \Delta \psi=\mu
$$

The Laplace equation only determines the solution up to an additive constnat and the particular solution $\psi_{\mu}$ above is singled out by the normalizing condition that $\psi(z)-\log |z| \rightarrow 0$ as $|z| \rightarrow \infty$.

A potential $\psi_{K}$ for the equilibrium measure $\mu_{K}$ of $K$ :

$$
\mu_{K}=\frac{1}{4 \pi} \Delta \psi_{K}
$$

can be obtained by a classical envelope construction (due to Perron):

$$
\psi_{K}(z)=\sup \left\{\psi: \quad \psi \operatorname{sh} \text { on } \mathbb{C}, \psi(z)=\log |z|^{2}+O(1)|z| \gg 1, \psi \leq 0 \text { on } K,\right\}
$$

where we recall that a function $\psi$ in $L_{l o c}^{1}$ is said to be subharmonic (sh) if it is (strongly) upper semi-continuous and $\Delta \psi \geq 0$, i.e. the distributional Laplacian of $\psi$ defines a measure on $\mathbb{C}$. By construction the potential $\psi_{K}$ integrates to zero against $\mu_{K}$ and thus has a different normalization than $\psi_{\mu_{K}}$. The envelope representation above can be deduced from the subdifferential (Euler-Lagrange) condition $\partial E(\mu) \geq 0$ at the minimizer $\mu_{K}$ combined with the domination principle for the Laplacian.

2.1.2. The weighted setting and elliptic Fekete points on the two-sphere. We also recall that the previous setup naturally extends to the more general weighted setting where the compact set $K$ is replaced by a weight function $\phi$, i.e. a lsc function on $\mathbb{C}$ assumed finite and continuous on a non-polar subset of $\mathbb{C}$. Then the point-wise norm of a degree $k$ polynomial is replaced by the weighted point-wise norm:

$$
\left|p_{k}(z)\right|_{k \phi}^{2}:=\left|p_{k}(z)\right|^{2} e^{-k \phi(z)}
$$

and the corresponding interpolation problem amounts to finding good points for interpolating degree $k$ polynomials, making interpolation stable with respect to the weighted sup-norms. Accordingly, the point-wise norm of the Vandermonde determinant is replaced by the weighted norm:

$$
\left|D\left(z_{1}, \ldots, z_{N}\right)\right|_{k \phi}^{2}:=\left|D\left(z_{1}, \ldots, z_{N}\right)\right|^{2} e^{-k \phi\left(z_{1}\right)} \cdots e^{-k \phi\left(z_{1}\right)}
$$

In the case when $\phi$ is the indicator function of a compact set $K$ the weighted setting thus reduces to the previous setting. Accordingly, the corresponding weighted Fekete points are the minimizers of

$$
E_{\phi}^{(N)}\left(z_{1}, \ldots z_{N_{k}}\right):=E^{(N)}\left(z_{1}, \ldots z_{N}\right)+N^{-1} \sum_{i=1}^{N} \phi\left(z_{i}\right)
$$


showing that the weight function $\phi$ plays the role of an exterior potential, from the physics point of view. In order to ensure the existence of weighted Feketete points the weight $\phi$ is assumed to have super logarithmic growth:

$$
\phi(z) \geq \log |z|^{2}+O(1), \quad|z| \gg 1
$$

In the critical case where equality holds above the support of the corresponding equlibrium measure $\mu_{\phi}$ may be non-compact, in general. The model case is when the weight is given by

$$
\phi_{F S}(z):=\log \left(1+|z|^{2}\right),
$$

that we shall refer to as the Fubini-Study weight (with an eye towards the higher dimensional setting considered below). Then $E_{\phi}^{(N)}\left(z_{1}, \ldots z_{N}\right)$ identifies, under sterographic projection, with the standard logarithmic energy on the two-sphere, which is invariant under the action of the isometry group $O(3)$ of the two-sphere. By symmetry the corresponding equilibrium measure $\mu_{\phi_{F S}}$, emerging when $N \rightarrow \infty$, thus identifies with the standard $O(3)$ - invariant probability measure on the two-sphere. But the problem of locating the corresponding minimizers (called elliptic Fekete points) is extremely challenging for a finite $N$. For example, Smale asked in the year of 2000, in his list of mathematical problems for the next century [106, Problem 7], for an algorithm which can locate configuration of points with almost minimal energy $E_{\phi}^{(N)}$ (in a polymomial time, tolarating an error of the order $O\left(N^{-2} \log N\right)$.

2.2. Fekete points in $\mathbb{C}^{n}$ and the pluripotential equilibrium measure. Let now $K$ be a compact subset of $\mathbb{C}^{n}$ and denote by $\mathcal{P}_{k}\left(\mathbb{C}^{n}\right)$ the space of all polynomials on $\mathbb{C}^{n}$ with total degree at most $k$. This is a complex vector space of dimension

$$
N_{k}:=\operatorname{dim} \mathcal{P}_{k}\left(\mathbb{C}^{n}\right)=\frac{k^{n}}{n !}+o\left(k^{n}\right)
$$

Fekete points for $K$ are defined, precisely as in the one-dimensional setting above, as maximizers of the absolute value of the determinant of the corresponding evaluation map from $\mathcal{P}_{k}\left(\mathbb{C}^{n}\right)$ to $\mathbb{C}^{N_{k}}$ (such points are also called extremal points or extremal systems in the literature). Fixing a base $e_{j}$ in $\mathcal{P}_{k}\left(\mathbb{C}^{n}\right)$ consisting of multinomials we will use the same notation $D^{\left(N_{k}\right)}\left(z_{1}, \ldots z_{N_{k}}\right)$ for the higher dimensional incarnation of the Vandermonde determinant, defined by formula 2.2 .

Given a general configuration $\left(z_{1}, \ldots z_{N}\right)$ of $N$ points we will denote by $\delta_{N}$ the corresponding discrete probability measure on $\mathbb{C}^{n}$, called the empirical measure:

$$
\delta_{N}:=\frac{1}{N} \sum_{i=1}^{N} \delta_{z_{i}} .
$$

In the light of Theorem 2.1 it is natural to ask

(1) Does the sequence of empirical measure $\delta_{N_{k}}$ corresponding to a sequence $\left(z_{1}, \ldots, z_{N}\right)$ of Fekete points on $K$ admit a unique weak limit $\mu_{K}$ in the space $\mathcal{M}_{1}(K)$ of probability measures on $K$ ?

(2) If so, is there a direct analytic characterization of $\mu_{K}$ ?

A suitable generalization to $\mathbb{C}^{n}$ of the equilibrium measure appearing in Theorem 2.1 would be a natural analytic candidate for $\mu_{K}$. However, for various reasons the naive generalization to $\mathbb{C}^{n}$ of the logarithmic energy $E(\mu)$, defined by formula 2.3 , is not the right object to look at in the higher dimensional setting of $\mathbb{C}^{n}$. In the seventies Siciak [102, 103] and Zaharjuta [109] 
instead introduced a natural higher dimensional generalization of the equilibrium potential $\psi_{K}$, by generalizing the envelope expression 2.8

$$
\psi_{K}(z)=\sup _{\psi \in \mathcal{L}\left(\mathbb{C}^{n}\right)}\{\psi(z): \psi \leq 0 \text { on } K\}
$$

where $\mathcal{L}\left(\mathbb{C}^{n}\right)$ denotes the Lelong class, i.e. the class of all plurisubharmonic (psh) functions $\psi$ on $\mathbb{C}^{n}$ with sublogarithmic growth:

$$
\mathcal{L}\left(\mathbb{C}^{n}\right):=\left\{\psi: \psi \mathrm{psh}, \psi(z) \leq \log |z|^{2}+O(1),|z| \gg 1\right\}
$$

The subclass of all psh $\psi$ with logarithmic growth is is denoted by $\mathcal{L}_{+}\left(\mathbb{C}^{n}\right)$, i.e. $\psi$ is psh and

$$
\psi(z)=\log |z|^{2}+O(1)|z| \gg 1
$$

We recall that a function $\psi$ on $\mathbb{C}^{n}$ is plurisubharmonic (psh) if it is usc and subharmonic along complex lines. These functions form the backbone of pluripotential theory and appear naturally in polynomial approximation theory. Indeed, if $p_{k}(z)$ is a degree $k$ polynomial on $\mathbb{C}^{n}$, then $k^{-1} \log \left|p_{k}(z)\right|$ is psh and in the Lelong class $\mathcal{L}\left(\mathbb{C}^{n}\right)$.

The function $\psi_{K}$ is bounded precily when $K$ is non-pluripolar, i.e not locally contained in the $-\infty$-set of a psh function. We will then identify $\psi_{K}$ with its upper-semi continuous regularization, which defines an element in $\mathcal{L}_{+}\left(\mathbb{C}^{n}\right)$. The pluripotential equilibrium measure of a compact set $K \subset \mathbb{C}^{n}$, assumed non-pluripolar, is then defined by generalizing formula 2.7 .

$$
\mu_{K}:=M A\left(\psi_{K}\right),
$$

where $M A$ denotes the complex Monge-Ampère operator, which is the fully-nonlinear operator generalization the linear Laplace operator to pluripotential theory:

$$
M A(\psi):=\left(\frac{i}{2 \pi} \partial \bar{\partial} \psi\right)^{n}=\frac{1}{\pi^{n}} \operatorname{det}\left(\frac{\partial^{2} \psi}{\partial z_{i} \partial \bar{z}_{j}}\right) d \lambda,
$$

when $\psi$ is smooth and the extension to locally bounded psh is due to Bedford-Taylor (see Section 3.1). The measure $\mu_{K}$ is supported on $K$ (as follows from the maximum/domination principle) and defines a probability measure on $K$. With this pluripotential generalization of the classical equilibrium measure in hand it was conjectured by Siciak [104] that the analog of Theorem 2.1 holds for any non-pluricompact subset of $K$ [104]. This was confirmed in [27]:

Theorem 2.3. Assume that $K$ is a compact subset of $\mathbb{C}^{n}$ which is non-pluripolar. Let $\left(z_{1}, \ldots z_{N_{k}}\right) \in K^{N_{k}}$ be a sequence of Fekete points on $K$. Then the corresponding empirical measures $\delta_{N}$ converge, as $k \rightarrow \infty$, to the pluripotential equilibrium measure $\mu_{K}$ of $K$, in the weak topology of measures on $K$.

The proof of the theorem in the higher dimensional setting is very different from the proof in the classical one-dimensional setting, as the Vandermonde determinant cannot be factorized when $n>1$. This means that there is no tractable expression for the corresponding higherdimensional analog of the discrete logarithmic energy:

$$
E^{\left(N_{k}\right)}\left(z_{1}, \ldots z_{N_{k}}\right):=-\frac{1}{N_{k} k} \log \left|D^{\left(N_{k}\right)}\left(z_{1}, \ldots z_{N_{k}}\right)\right|^{2}
$$

that we shall refer as the determinantal energy. Still, the proof in [27], which builds on [26], naturally leads to a notion of pluricomplex energy $E(\mu)$ (further studied in [28]). In fact, as shown in[18], the proof in [27] can be upgraded to give the more precise result

$$
E^{\left(N_{k}\right)} \rightarrow E, \quad k \rightarrow \infty
$$


in the sense of Gamma-convergence on the space $\mathcal{M}_{1}(K)$ of probability measures on $K$, where $E$ is a functional on $\mathcal{M}_{1}(K)$, whose unique minimizer is the equilibrium measure $\mu_{K}$ (see [100] for a different proof of the Gamma-convergence in the one-dimensional case). This notion of convergence, introduced by Di Georgi, plays a prominent role in the calculus of variations. Its definition ensures that any minimizer of $E^{\left(N_{k}\right)}$ converges to the unique minimizer of $E$. The functional $E$ coincides with the pluricomplex energy introduced in [28]. It may be explicitely expressed in terms of the potential $\psi_{\mu}$ of the measure $\mu$ i.e. the unique (modulo constants) solution of the complex Monge-Ampère equation

$$
M A(\psi)=\mu, \quad \psi \in \mathcal{L}_{+}\left(\mathbb{C}^{n}\right)
$$

known as the Calabi-Yau equation in the complex (Kähler) geometry literature (generalizing the Laplace equation 2.6. In fact, if $\mu$ is sufficently regular then $-\psi_{\mu}$ can be viewed as the differential (gradient) of $E(\mu)$ (see Section 3.6).

Example 2.4. In the case when $K$ is a symmetric convex body in $\mathbb{R}^{n} \Subset \mathbb{C}^{n}$ the equilibrium measure $\mu_{K}$ can be computed explicitely [9. Comparing with the one-dimensional case reveals that its density is comparable to inverse of the squar-root of the distance $d(x, \partial K)$ to the boundary.

The proof of Theorem 2.3 in [27] is given in the general complex geometric setting of a big line bundle $L$ over a compact complex manifold $X$. Then the role of the space $\mathcal{P}_{k}\left(\mathbb{C}^{n}\right)$ is played by the space $H^{0}\left(X, L^{\otimes k}\right)$ of global holomorphic sections and the role of the weight function $\phi$ is played by a (possibly singular) metric on the line bundle $L$. From this point of view Theorem 2.3 appears as the special case when $L \rightarrow X$ is the hyperplane line bundle $\mathcal{O}(1) \rightarrow \mathbb{P}^{n}$ over the $n$-dimensional complex projective space $\mathbb{P}^{n}$, compactifying $\mathbb{C}^{n}$. In this case the corresponding metric on the restricted line bundle $L \rightarrow K$ is the trivial one, but the more general weighted version of Theorem 2.3 also follows by using a general metric $\phi$ on $L$. In Section 5 we will explain the proof in the technically simpler case where $L$ is a positive line bundle, i.e. $(X, L)$ is a polarized manifold, which is enough for the application to $\mathbb{C}^{n}$ (see Theorem 5.31). But the case when $L$ is big allows one, in particular, to also cover the case when $X$ is a singular variety.

Example 2.5. Another concrete application of Theorem 5.3 is to the case when $K$ is taken as a compact real algebraic variety in $\mathbb{R}^{n+1}$ and $\mathcal{P}_{k}$ as the restriction to $K$ of the space of all polynomials on $\mathbb{R}^{n+1}$ of total degree at most $k$. Then the analog of Theorem 2.3 follows from Theorem 5.3, applied to the complexification $X$ in $\mathbb{P}^{n+1}$ of $K$. For example, $K$ may be taken as the unit-sphere $S^{n} \Subset \mathbb{R}^{n+1}$. Then $\mathcal{P}_{k}$ is the space of all sperical polynomials of degree at most $k$, i.e. the space of all spherical harmonics band-limited to $[0, k]$. The corresponding Fekete points are often refererred to as extremal systems (see [105] where numerical approximations are computed on the two-sphere, $n=2$, when $k \leq 191$, which corresponds to $N_{k}=36864$ points). By symmetry the pluripotential equilibrium measure $\mu_{K}$ is the $O(n+1)$ invariant probability measure on the sphere.

An interesting feature of the proof of the general version of Theorem 2.3, for a polarized complex manifold $(X, L)$ (Theorem 5.3), is that it essentially reduces the proof of Theorem 2.3 for a compact subset $K$ of $\mathbb{C}^{n}$ to the weighted setting where $K$ is replaced by a smooth weight $\phi$ on $\mathbb{C}^{n}$ with logarithmic growth (i.e. equality holds in formula 2.10). Then the analog of $\psi_{K}$ is the weighted extremal function $\psi_{\phi}$ defined by the upper semi-continuous regularization of 
the envelope

$$
\psi_{\phi}(z):=\sup _{\psi \in \mathcal{L}\left(\mathbb{C}^{n}\right)}\left\{\psi(z): \psi \leq \phi \text { on } \mathbb{C}^{n}\right\}
$$

and the correponding weighted equilibrium measure is the probability measure defined as

$$
\mu_{\phi}:=M A\left(\psi_{\phi}\right)
$$

More generally, Theorem 5.3 applies to the general setting where one is given a closed nonpluripolar subset $K$ of $\mathbb{C}^{n}$ and a continuous weight function $\phi$ on $K$ with logarithmic growth. Then the corresponding extremal function $\psi_{(K, \phi)}$ is defined by

$$
\psi_{(K, \phi)}(z):=\sup _{\psi \in \mathcal{L}\left(\mathbb{C}^{n}\right)}\{\psi(z): \psi \leq \phi \text { on } K\}
$$

2.3. Statistical mechanics and the emergence of twisted Kähler-Einstein metrics at positive temperature. Comparing with the one-dimensional situation it is tempting to think of Fekete points on a compact subset $K$ of $\mathbb{C}^{n}$ (and more generally, nearly optimal interpolation nodes) as interacting particles confined to $K$, forming a microscopic equilibrium state. In [18, 21] this analogy is pushed further by introducing temperature (and thus randomness) into the picture. To explain this let us first recall the general statistical mechanical setup. Consider a system of $N$ identical particles on a space $X$ interacting by a microscopic interaction energy $H^{(N)}\left(x_{1}, . . x_{N}\right)$ (the Hamiltonian) on $X^{N}$ assumed symmetric (as the particles are identical) and lower semi-continuous. Given a measure $d V$ on $X$ the distribution of particles, in thermal equilibrium at a fixed temperature $\left.\beta^{-1} \in\right] 0,-\infty[$, is described by the corresponding Gibbs measure. This is the symmetric probability measure on $X^{N}$ defined by

$$
\mu_{\beta}^{(N)}:=\frac{1}{Z_{N, \beta}} e^{-\beta H^{(N)}\left(x_{1}, \ldots, x_{N}\right)} d V^{\otimes N}, \quad Z_{N, \beta}:=\int_{X^{N}} e^{-\beta H^{(N)}\left(x_{1}, \ldots, x_{N}\right)} d V^{\otimes N}
$$

where the normalizing constant $Z_{N, \beta}$ is called the partition function and is assumed to be finite (which is automatic if $X$ is compact, as $H^{(N)}$ is assumed lsc and hence bounded from below). The probability space $\left(X^{N}, \mu_{\beta}^{(N)}\right)$ is called the canonical ensemble in the physics literature. The empirical measure

$$
\delta_{N}:=\frac{1}{N} \sum_{i=1}^{N} \delta_{x_{i}}: \quad X^{N} \rightarrow \mathcal{M}_{1}(X)
$$

can now be viewed as a random measure, i.e. a random variable on $\left(X^{N}, \mu_{\beta}^{(N)}\right)$ taking values in the space of probability measures on $X$. Heuristic reasoning, inspired by mean field theory in physics suggests the following "Mean Field Approximation": the random measures $\delta_{N}$ converge in law towards a deterministic limit $\mu_{\beta}$, in the many particle limit $N \rightarrow \infty$ (i.e. the random measure $\delta_{N}$ is well approximated by $\mu_{\beta}$ ) under the following assumptions:

- The microscopic "energy per particle"

$$
E^{(N)}\left(x_{1}, \ldots x_{N}\right):=\frac{1}{N} H^{(N)}\left(x_{1}, \ldots, x_{N}\right)
$$

converges, in the many particule limit $N \rightarrow \infty$, towards a "macroscopic energy" $E(\mu)$ on the space $\mathcal{M}_{1}(X)$ in an apprioate sense (to be specified!) 
- The corresponding macroscopic free energy functional $F_{\beta}$ on $\mathcal{M}_{1}(X)$ admits a unique minimizer, to wit $\mu_{\beta}$ :

$$
F_{\beta}(\mu):=E(\mu)-\beta^{-1} S_{d V}(\mu),
$$

where $S_{d V}(\mu)$ is the "physical entropy" of $\mu$ relative to $d V$ (see Remark 4.11).

In order to make the "Mean Field Approximation" into a mathematically precise statement the meaning of the convergence in the first point above needs to be specified (see Section 4.4 for further discussion). The main two classes of cases where the Mean Field Approximation has been rigorously established is in the

- "Regular case": $X$ is compact and $E^{(N)}$ is continuous, uniformly wrt $N$ 60].

- "Linear case": $E^{(N)}$ is a sum of the form 2.4 with a lsc pair interaction potentail $g \in L^{1}\left(X^{2}, d V^{\otimes 2}\right)[77,42]$

In the latter case a trunctation argument can be employed to essentially reduce the problem to the "regular case" (a similar argument applies to a finite sum of $m$-point interactions [20]). However, in the present setting $E^{(N)}$ (as defined by formula 2.14) is both very non-linear (when $n>1$ ) and singular. What saves the situation is that $E^{(N)}$ is superharmonic so that the following key result in [18] can be applied:

Theorem 2.6. Assume that $X$ is a compact Riemannian manifold without boundary and that the sequence $\frac{1}{N} H^{(N)}\left(x_{1}, \ldots, x_{N}\right)$ Gamma converges to a functional $E(\mu)$ on $\mathcal{M}_{1}(X)$. If moreover, $H^{(N)}$ is uniformly quasi-superharmonic, i.e. there exists a constant $C$ such that for all $N$

$$
\Delta_{x_{i}} H^{(N)}\left(x_{1}, x_{2}, \ldots x_{N}\right) \leq C \quad X^{N}
$$

then the laws of the random measures $\delta_{N}$ on $\left(X^{N}, \mu_{\beta}^{(N)}\right)$ satisfy a Large Deviation Principle $(L D P)$ with speed $\beta N$ and rate functional $F_{\beta}(\mu)-C_{\beta}$, where

$$
C_{\beta}=\inf _{\mathcal{M}_{1}(X)} F_{\beta}
$$

In particular, if $E$ is convex and $\beta>0$ then $\delta_{N}$ converges in law towards the unique minimizer $\mu_{\beta}$ of $F_{\beta}$.

The general notion of a LDP is recalled in Section 4.2.1. But loosely speaking, the LDP implies that the risk that the empirical measure $\delta_{N}$ deviates from the minimizer $\mu_{\beta}$ is exponentiall small, as $N \rightarrow \infty$, which implies convergence in law towards $\mu_{\beta}$. The idea of the proof of the previous theorem is to apply geometric analysis and comparison geometry to the configuration space of $N$ points on $X$, viewed as a Riemannian orbifold (see Section 5).

2.3.1. Random interpolation nodes. In the present setting, where $N$ is taken as the sequence $N_{k}$ of integers defined by formula 2.11, we take the Hamiltonian to be

$$
H^{\left(N_{k}\right)}\left(z_{1}, \ldots z_{N_{k}}\right)=-\frac{1}{k} \log \left|D^{\left(N_{k}\right)}\left(z_{1}, \ldots z_{N_{k}}\right)\right|_{k \phi}^{2}
$$

for a given weight function $\phi$ on $\mathbb{C}^{n}$, assumed continuous (which, from the statistical mechanics point of view, plays the role of an exterior potential, just as in the one-dimensional case 
discussed above). Fixing also a continuous volume form $d V$ on $\mathbb{C}^{n}$ the corresponding Gibbs measure is thus given by the following propobability measure on $\left(\mathbb{C}^{n}\right)^{N}$ :

$$
\mu_{\beta}^{\left(N_{k}\right)}=\frac{1}{Z_{N, \beta}}\left|D^{\left(N_{k}\right)}\left(z_{1}, \ldots, z_{N_{k}}\right)\right|_{k \phi}^{2 \beta / k} d V^{\otimes N},
$$

(which, as explained in Section 5.2, can be viewed as a deformation of a determinantal random point process). Denoting by $d \lambda$ Lesbesgue measure on $\mathbb{C}^{n}$ we will say that the given "weighted measure" $(\phi, d V)$ is admissble wrt $\beta$ if there exists $\epsilon>0$ such that

$$
\phi-\beta^{-1} \log (d V / d \lambda) \geq(1+n / \beta+\epsilon) \log |z|^{2}|z| \gg 1
$$

and $\phi$ has at most iterated exponential growth, i.e. there exists a positive constant $C$ and a positive integer $m$ such that

$$
\phi(z) \leq C e^{(m)}(z)
$$

where $e^{(m)}(t)$ denotes the $m$ times iterated exponential function. The first assumption ensures that $Z_{N, \beta}$ is finite. The second one is of a technical nature and can, most likely, be removed (but it is satisifed for any reasonable $\phi$ ). In particular, if $d V=d \lambda$ and $\phi$ has strictly superlogarithmic growth, i.e. there exists $\epsilon>0$ such that

$$
\phi \geq(1+\epsilon) \log |z|^{2}+O(1),|z| \gg 1,
$$

then the first assumption is satisfied, for $\beta$ sufficently large. Note that we might as well have assumed that $d V=d \lambda$ since only the combination $e^{-\beta \phi} d V$ appears in the definition of $\mu_{\beta}^{\left(N_{k}\right)}$. However, when investigating the "infinite temperature limit" $(\beta \rightarrow 0)$ it will be necessary to consider the case when $d V$ is a probability measure (see Section 2.4 ).

In view of the connection to interpolation nodes we will refer a random configuration $\left(x_{1}, \ldots, x_{N}\right)$ in the probability space $\left(\mathbb{C}^{n}, \mu_{\beta}^{\left(N_{k}\right)}\right)$ as " $N_{k}$ random interpolation nodes at inverse temperature $\beta$ ". Since $H^{\left(N_{k}\right)}\left(z_{1}, \ldots z_{N_{k}}\right)$ is strongly repulsive in the sense that $H^{\left(N_{k}\right)}\left(z_{1}, \ldots z_{N_{k}}\right) \rightarrow$ $\infty$ if two points merge, random interpolation nodes behave as repulsive particles.

Using a compactification argument to replace the non-compact space $\mathbb{C}^{n}$ with the complex projective space $\mathbb{P}^{n}$ the following stochastic analog of Theorem 2.7 will be deduced from Theorem 2.6 .

Theorem 2.7. Given a weighted measure $(\phi, d V)$ which is admissble with respect to $\beta$, the random measures $\delta_{N_{k}}$ on the probability spaces $\left(\left(\mathbb{C}^{n}\right)^{N}, \mu_{\beta}^{\left(N_{k}\right)}\right)$ converge in law , as $N_{k} \rightarrow \infty$, to the unique minimizer $\mu_{\beta}$ of the following free energy type functional on the space $\mathcal{M}_{1}\left(\mathbb{C}^{n}\right)$ of probability measures on $\mathbb{C}^{n}$ :

$$
F_{\phi, \beta}(\mu):=E_{\phi}(\mu)-\beta^{-1} S_{d V}(\mu),
$$

where $E_{\phi}(\mu)$ is the weighted pluricomplex energy of $\mu$ (given by $E(\mu)+\int \phi \mu$ when $\mu$ has compact support). Equivalently,

$$
\mu_{\beta}=e^{\beta\left(\psi_{\beta}-\phi\right)} d V
$$

where $\psi_{\beta}$ is the unique solution of the complex Monge-Ampere equation

$$
M A(\psi)=e^{\beta(\psi-\phi)} d V
$$


for a function $\psi \in \mathcal{L}_{+}\left(\mathbb{C}^{n}\right)$. Moreover,

$$
-\lim _{k \rightarrow \infty} \frac{1}{k N_{k}} \log \int_{\mathbb{C}^{n N_{k}}}\left|D\left(z_{1}, \ldots, z_{N_{k}}\right)\right|_{k \phi}^{2 \beta / k} d V^{\otimes N}=\inf _{\mu \in \mathcal{M}_{1}\left(\mathbb{C}^{n}\right)} F_{\phi, \beta}(\mu):=C_{\beta}
$$

More precisely, the laws of the random measures $\delta_{N}$ satisfy a Large Deviation Principle (LDP) on $\mathcal{M}_{1}\left(\mathbb{C}^{n}\right)$ with speed $\beta N$ and rate functional $F_{\phi, \beta}(\mu)-C_{\phi, \beta}$.

Note that the deterministic setting, exposed in the previous section, appears (formally) in the "zero temperature case", i.e. when $\beta=\infty$, since $\mu_{\beta}^{(N)}$ then localizes on the set of minima of $H^{(N)}$, i.e. on the maximizers of the weighted Vandermonde determinant. One motivation for increasing the temperature, i.e. introducing randomness into the picture, comes from Monte Carlo methods, as used extensively in scientific computing [87] (see [39, 84, 85] for the logarithmic case of elliptic Fekete points on the two-sphere). These are stochastic optimization methods that can be used for locating approximate minima of a given function $H^{(N)}$, by fixing a small temperature, i.e. a large $\beta$ (the corresponding Gibbs measure typically arises as the stationary measure for the stochastic gradient flow of $H^{(N)}$, as explained in Section [7.3).

Theorem 2.7 applies (and so does Theorem 2.6) to the more general situation when the inverse temperature $\beta_{N}$ is allowed to dependent on $N$ as long as its large- $N$ limit exists:

$$
\lim _{N \rightarrow \infty} \beta_{N}:=\beta \in[0, \infty]
$$

In fact, the proof is considerably simpler when $\beta=\infty$ and the result then applies more generally to measures $d V$ satisfying a Bernstein-Markov property (see formula 2.33 below). This leads to three different regimes (phases):

- The zero-temperature regime: $\beta=\infty$ (then $\mu_{\beta}$ minimizes the energy $E_{\phi}$ )

- The positive temperature regime: $\beta \in] 0, \infty\left[\right.$ (then $\mu_{\beta}$ minimizes the free energy $F_{\beta, \phi}$

- The infinite temperature regime: $\beta=0$ (then $\mu_{\beta}$ maximizes entropy $\left.S_{d V}\right)$

Example 2.8. The standard quadratic weight $\phi(z):=|z|^{2}$ is admissble for any $\beta$ when $d V=d \lambda$. This is equivalent to the non-weighted setting $\phi=0$, but with $d V$ taken as a Gaussian measure. Thus, for $\beta \in] 0, \infty\left[\right.$ the measure $\mu_{\beta}$ interpolates between the Gaussian measure at $\beta=0$ and the normalized Lesbegue measure on the unit-ball $B \subset \mathbb{C}^{n}$ at $\beta=\infty$ (compare Theorem 2.15). This "cross-over" behaviour has recently been studied when $n=1$ in [31, 2].

Remark 2.9. Establishing the LDP in Theorem 2.7 for a fixed weight $\phi$ is, in fact, equivalent to establishing the logarithmic asymptotics of the corresponding partition functions for all weights $\phi$ (formula 2.27); see Step 2 in the proof of Theorem 2.7. In other words, establishing the LDP in question is equivalent to computing the leading logarithmic asymptotics of the weighted $L^{p_{k}}$-norms of the Vandermonde determinant $D^{\left(N_{k}\right)}$, as $k \rightarrow \infty$ with $p_{k} k / 2 \rightarrow \beta$. Interestingly, in the case when $n=1$ such $L^{p}$-norms have been computed explicitely for all fixed $N$ and $p$ for some particular weighted measures $(\phi, d V)$; see the survey [62], explaining the links to the Selberg integral, random matrix theory and representation theory.

2.3.2. Complex geometry. The main motivation for increasing the temperature does not come from interpolation theory, but rather from complex geometry (and then the temperature need not be small). Indeed, when $\phi$ is smooth, the function $\psi_{\beta}$ determined by the measure $\mu_{\beta}$ by

$$
\psi_{\beta}:=\beta^{-1} \log \frac{\mu_{\beta}}{d V e^{-\phi}}
$$


(appearing in formula 2.25) is the Kähler potential of a Kähler metric

$$
\omega_{\beta}:=\frac{i}{2 \pi} \partial \bar{\partial} \psi_{\beta}
$$

which defines a twisted Kähler-Einstein metric on $\mathbb{C}^{n}$ :

$$
\operatorname{Ric} \omega_{\beta}+\beta \omega_{\beta}=\tau,
$$

where the twisting form $\tau$ is explicetly expressed is terms of the given data $(\beta, \phi, d V$ ) (see Example 3.3.

Kähler-Einstein metics (i.e. the case when $\eta=0$ ) and their twisted generalization play a key role in current Kähler geometry, in particular in connection to the Yau-Tian-Donaldson conjecture [54. Thus Theorem 2.7furnishes a probabilistic approach for constructing solutions $\omega_{\beta}$ to the twisted Kähler-Einstein equation 2.30, by first sampling configurations of $N$ points with respect to the probability measure on $\left(\mathbb{C}^{n}\right)^{N}$ defined by formula 2.22 in order to obtain $\mu_{\beta}$ as $N \rightarrow \infty$ (with overwhelming probability) and then taking the logarithm to obtain the Kähler potential $\psi_{\beta}$ defined by formula 2.29. Moreover, the rate functional $F_{\beta}$ may in this context be identified with the twisted K-energy functional in Kähler geometry [15].

The convergence in law in Theorem 2.7 implies, in particular, that the expectations $\mathbb{E}\left(\delta_{N_{k}}\right)$ of the empirical measure $\delta_{N_{k}}$ converge weakly towards $\mu_{\beta}$, as measures on $\mathbb{C}^{n}$. By symmetry $\mathbb{E}\left(\delta_{N_{k}}\right)$ may be explicitely expressed as the measure

$$
\nu_{k}:=\frac{\int_{\left(\mathbb{C}^{n}\right)^{N-1}}\left|D\left(\cdot, z_{2} \ldots, z_{N_{k}}\right)\right|_{k \phi}^{2 \beta / k} d V^{\otimes N}}{\int_{\left(\mathbb{C}^{n}\right)^{N}}\left|D\left(z_{1}, z_{2} \ldots, z_{N_{k}}\right)\right|_{k \phi}^{2 \beta / k} d V^{\otimes N}}
$$

on $\mathbb{C}^{n}$. Theorem 2.7 thus implies the following convergence of the explicit sequence $\nu_{k}$ of measures on $\mathbb{C}^{n}$ and its logarithms:

Corollary 2.10. Given a weighted measure $(\phi, d V)$ which is admissble with respect to $\beta$, the sequence of measures converge in the weak topology of measures on $\mathbb{C}^{n}$ towards $\mu_{\beta}$. As a consequence, the corresponding sequence of Kähler potentials

$$
\psi_{\beta}^{\left(N_{k}\right)}:=\frac{1}{\beta} \log \frac{\nu_{k}}{d V e^{-\phi}}
$$

converge in $L_{\text {loc }}^{1}\left(\mathbb{C}^{n}\right)$ towards the Kähler potential $\psi_{\beta}$ of a twisted Kähler-Einstein metric $\omega_{\beta}$ (solving the equation 2.30).

As will be explained in Section 2.5 the convergence of $\psi_{\beta}^{\left(N_{k}\right)}$ towards $\psi_{\beta}$ may be interpreted as a "positive temperature analogy" of a classical convergence result of Siciak.

The existence of solutions to Monge-Ampère equations of the form 2.26] on a compact Kähler manifold $X$ was first established by Aubin [5] and Yau [110, when $\beta>0$. The case $\beta=0$ (i.e. the Calabi-Yau equation) was settled in Yau's solution of the Calabi conjecture [110]. Interestingly, the minus the "inverse temperature" $-\beta$ coincides with the deformation parameter in Aubin's continuity method for constructing solutions to the equation 2.26 on a compact Kähler manifold $X$ for $\beta$ negative [5]. This is motivated by the Yau-Tian-Donaldson conjecture concerning the existence of Kähler-Einstein metrics with positive Ricci curvature, which very recently has been settled for smooth compact Fano manifolds $X$ (see the survey [54]). From a statistical mechanical point of view the case $\beta<0$ introduces an additional fourth phase, complementing the three phases described above. In practise, switching the sign 
of $\beta$ equivalently corresponds to switching the sign of the corresponding interaction energy, thus replacing the repulsive interaction energy $E^{(N)}$ with the opposite attractive energy

$$
E_{\text {attr }}^{(N)}\left(z_{1}, \ldots, z_{N}\right):=\frac{1}{N_{k} k} \log \left|D^{\left(N_{k}\right)}\left(z_{1}, \ldots z_{N_{k}}\right)\right|^{2}
$$

at positive inverse temperature $\gamma=-\beta$. This attractive setting appears to be extremely challenging (see Section 7.4).

2.4. The zero and infinite temperature limits and the Calabi-Yau equation. Let us come back to the original "zero temperature regime" $(\beta=\infty)$ and explain how it reappears when $\beta \rightarrow \infty$. Since the measure $\mu_{\beta}$, emerging in the large $N$-limit described in Theorem 2.7. is a minimizer of the free energy functional $F_{\beta, \phi}$ it is not hard to see that $\mu_{\beta}$ converges, as $\beta \rightarrow 0$, to the minimizer of $E_{\phi}$, which coincides with weighted equilibrium measure $\mu_{\phi}$ (formula 2.18) when $\beta \rightarrow \infty$. Moreover, the corresponding result also holds on the level of potentials. In other words, the corresponding Kähler potentials $\psi_{\beta}$ (formula 2.17) converge to the corresponding extremal function $\psi_{\phi}$, which is $C^{1,1}$-smooth when $\phi$ is smooth (but not $C^{2}$-smooth, in general). More precisely:

Theorem 2.11. Assume that $\phi$ has strictly super-logarithmic growth (formula 2.24), that $\phi$ is in $C_{\text {loc }}^{2}\left(\mathbb{C}^{n}\right)$ and that $d V$ has a positive density in $C_{\text {loc }}^{2}\left(\mathbb{C}^{n}\right)$. Then $\psi_{\phi}$ is in $C_{\text {loc }}^{1,1}\left(\mathbb{C}^{n}\right)$ and

$$
\lim _{\beta \rightarrow \infty} \psi_{\beta}=\psi_{\phi}
$$

in the Hölder space $C_{\text {loc }}^{1, \alpha}\left(\mathbb{C}^{n}\right)$ for any $\alpha<1$.

The result follows from a straight-forward modification of the proof in the setting of polarized manifolds [21]. The $C^{1,1}$-regularity implies that

$$
\mu_{\phi}=1_{S_{\phi}} M A(\phi)
$$

which typically has a sharp discontinuity across boundary points of the support $S_{\phi}$, which is a compact subset of $\mathbb{C}^{n}[12$. This should be contrasted with the fact that for any finite $\beta$ the support of the Monge-Ampère measure $M A\left(\psi_{\beta}\right)$ is all of $\mathbb{C}^{n}$. Thus, from a statistical mechanical point of view, the "zero-temperature limit" $\beta \rightarrow \infty$ is reminiscent of a second order "gas-liquid" phase transition.

More generally, one can consider the situation where the volume form $d V$ is replaced by a general measure on $\mathbb{C}^{n}$, that we shall still denote by $d V$. We denote by $K$ the support of $d V$, which, for simplicity will be assumed to be compact. From the pluripotential point of view a natural condition is that the measure $d V$ is determining for $(K, \phi)$, i.e.

$$
\psi \leq \phi \text { almost everywhere wrt } d V \Longrightarrow \psi \leq \phi \text { on } K
$$

The following result appears to be new, also when $n=1$; see [24], where the setting Coulomb gases is considered (the notion of convergence in energy, coinciding with Sobolev $H^{1}$-convergence when $n=1$, is recalled in Section [6).

Theorem 2.12. Let $\phi$ be a continuous function on $\mathbb{C}^{n}$ and assume that $d V$ does not charge pluripolar subsets and is determining wrt $(K, \phi)$. Then, as $\beta \rightarrow \infty$,

$$
\psi_{\beta} \rightarrow \psi_{(K, \phi)}
$$


in energy. Moreover, if $d V$ is determining wrt $(K, \phi)$ for any $\phi \in C(K)$, then

$$
F_{\beta}:=E-\beta^{-1} S_{d V} \rightarrow E
$$

in the sense of Gamma-convergence on $\mathcal{M}_{1}(K)$. 1

As pointed out in Section 2.5 the convergence of $\psi_{\beta}$ towards Siciak's weighted extremal function $\psi_{(K, \phi)}$ (formula 2.19) can be interpreted as a transcendental analog of a classical result of Siciak [103] and its $L^{2}$-version in [35].

The Gamma-convergence in the previous theorem may be equivalently formulated as the following approximation property of independent interest (see [24]):

Corollary 2.13. Let $\mu_{0}$ be a measure on $\mathbb{C}^{n}$ with compact support $K$ and assume that that $\mu_{0}$ does not charge pluripolar subsets and is determining for $(K, \phi)$ for any $\phi \in C(K)$. Then, for any given probability measure $\mu$ on $K$ there exists a sequence $\mu_{j}$ of probability measures, absolutely continuous wrt $\mu_{0}$, converging weakly to $\mu$ and with the property that $E\left(\mu_{j}\right) \rightarrow E(\mu)$.

Remark 2.14. In the case when $n=1$ (or more generally, in the "linear setting" discussed in Section 4.17) this type of approximation property is used as a hypothesis to derive large deviation results for Gibbs measures in the "zero-temperature regime", where $\beta_{N} \rightarrow \infty$ [45, 56, 20, 67]. These connections are discussed in [24]. We note that when $\mu_{0}$ is supported in $\mathbb{R}^{n}$ $\mu_{0}$ is determining for $(K, 0)$ iff it is determining for $(K, \phi)$ for all $\phi \in C(K)$ (using that, by the Stone-Weierstrass theorem, $-\phi$ is the uniform limit of $\log \left|p_{k}\right|^{2}$ for some $\left.p_{k} \in \mathcal{P}_{k}\left(\mathbb{C}^{n}\right)\right)$.

In the opposite "infinite temperature limit", where $\beta \rightarrow 0$, one has to assume that $d V$ is a probability measure. Then, under appropriate growth and regularity assumptions on $d V$, it follows from well-known results that $\psi_{\beta}$ converges to a particular solution $\psi_{0}$ of the CalabiYau equation 2.16 with $\mu=d V$ (see [64]). In Section 5 we will show that the convergence holds in great generality, only assuming that the probability measure $d V$ has finite weighted pluricomplex energy $E_{\phi}$ :

Theorem 2.15. Let $d V$ be a probability measure on $\mathbb{C}^{n}$ and fix a continuous function $\phi$ on $\mathbb{C}^{n}$ with super logarithmic growth 2.10. Assume that $d V$ has finite weighted pluricomplex energy, $E_{\phi}(d V)<\infty$. Then the functions $\psi_{\beta}$ converge to $\psi_{0}$ in energy, as $\beta \rightarrow 0$, where $\psi_{0}$ is the unique finite energy solution in $\mathcal{L}\left(\mathbb{C}^{n}\right)$ to

$$
M A(\psi)=d V
$$

satisfying the normalization condition

$$
\int_{X}\left(\psi_{0}-\phi\right) d V=0
$$

2.5. Comparison with determinantal point processes, orthogonal polynomials and Bergman kernels. Consider now the case when the inverse temperature $\beta$ is taken to depend on the number of points $N_{k}$ as

$$
\beta_{N_{k}}=k
$$

\footnotetext{
${ }^{1}$ Conversely, if the Gamma-convergence holds, then $d V$ is determining wrt $(K, \phi)$ for any $\phi \in C(K)$, as shown in [24].
} 
which means that the density of the probability measure $\mu_{\beta_{N_{k}}}^{\left(N_{k}\right)}$ (formula 2.22) is a squared Vandermonde determinant. In this case $\mu_{\beta_{N_{k}}}^{\left(N_{k}\right)}$ may be alternatively expressed as

$$
\mu_{\beta_{N_{k}}}^{\left(N_{k}\right)}=\frac{1}{N !} \operatorname{det}\left(\mathcal{K}_{k}\left(x_{i}, x_{j}\right)\right)_{i, j \leq N_{k}} d V^{\otimes N_{k}}
$$

where $\mathcal{K}_{k}(z, w)=K_{k}(z, w) e^{-k \phi(z)} e^{-k \phi(w)}$ and $K_{k}$ is the Bergman reproducing kernel of the Hilbert space space $\mathcal{P}_{k}\left(\mathbb{C}^{n}\right)$, endowed with the the scalar product defined by the weighted $L^{2}-$ norm

$$
\left\|p_{k}\right\|_{L^{2}(\phi, d V)}:=\left(\int_{\mathbb{C}^{n}}\left|p_{k}\right|^{2} e^{-k \phi} d V\right)^{1 / 2}
$$

Concretely,

$$
K_{k}(z, w):=\sum_{i=1}^{N_{k}} p_{i}^{(k)}(z) \overline{p_{i}^{(k)}(w)}
$$

in terms of a fixed orthonormal base $\left\{p_{i}^{(k)}\right\}_{i=1}^{N_{k}}$ of polynomials in the Hilbert space $\mathcal{P}_{k}\left(\mathbb{C}^{n}\right)$. In probabilistic terminology this means that the probability measure $\mu_{\beta_{N_{k}}}^{\left(N_{k}\right)}$ then defines a determinantal random point process with $N_{k}$ particles on $\mathbb{C}^{n}$ (see Section 4.3 for background on determinantal point processes). From a statistical mechanical point this case falls into the "zero temperature regime" (see formula 2.28) and, as shown in [13], the convergence in law in Theorem 2.7 then holds more generally as soon as the weighted measure $(d V, \phi)$ satisfies a weighted Bernstein-Markov property with respect to the weighted set $(K, \phi)$. This means that for any given $\epsilon>0$ there exists a constant $C$ such that

$$
\sup _{K}\left|p_{k}\right|^{2} e^{-k \phi} \leq C e^{\epsilon k}\left\|p_{k}\right\|_{L^{2}(d V, \phi)}
$$

for all $p_{k} \in \mathcal{P}_{k}\left(\mathbb{C}^{n}\right)$ and positive integers $k$ (see [34] for a survey of the Bernstein-Markovproperty). Moreover, defining $\psi_{\beta_{N_{k}}}^{\left(N_{k}\right)}$ as in Corollary [2.10, we then have

$$
\psi_{\beta_{N_{k}}}^{\left(N_{k}\right)} \rightarrow \psi_{(K, \phi)}, \quad k \rightarrow \infty
$$

in $L^{\infty}\left(\mathbb{C}^{n}\right)$, where we recall that $\psi_{(K, \phi)}$ denotes Siciak's weighted extremal function for the weighted set $(K, \phi)$ (formula 2.19). Since, under appropriate conditions,

$$
\psi_{\beta} \rightarrow \psi_{(K, \phi)}, \quad \beta \rightarrow \infty
$$

(by Theorem 2.12) the convergence 2.34 can be interpreted as zero-temperature analog of Corollary 2.10. To see that the convergence 2.34 holds first observe that $\psi_{\beta_{N_{k}}}^{\left(N_{k}\right)}$ may be reexpressed as

$$
\psi_{\beta_{N_{k}}}^{\left(N_{k}\right)}:=k^{-1} \log K_{k}(z, z)=k^{-1} \log \sup \frac{\left|p_{k}\right|^{2}}{\left\|p_{k}\right\|_{L^{2}(\phi, d V)}^{2}}
$$

(see Section 4.3) which converges towards $\psi_{(K, \phi)}$ in $L^{\infty}\left(\mathbb{C}^{n}\right)$, as first shown in a different probabilistic setting of random polynomials in [35]. Indeed, by the assumed weighted BernsteinMarkov-property of $(d V, \phi)$ we may as well replace the $L^{2}$-norm in the right hand side of 
formula 2.35 with an $L^{\infty}$-norm on $K$. The $L^{\infty}$-convergence towards Siciak's extremal function then follows from a classical result of Siciak [103].

Remark 2.16. In the classical setting of orthogonal polynomials on $\mathbb{R}$, where $K_{k}(z, z)$ is usually called the Christoffel-Darboux kernel, asymptotics of $k^{-1} \log K_{k}(z, z)$ are closely related to the notion of " $k$ th root asymptotics of orthonormal polynomials" [107]. Moreover, when $\phi=0$ and $K$ is regular in the sense of potential theory, the Bernstein-Markov-condition on $d V$ is equivalent to the condition that $d V$ has regular asymptotic distribution in the sense of [107, Chapter 4]. The case $n=1$ also naturally appears in Random Matrix Theory, as recalled in Section 4.3 .

The discussion above suggests viewing the function $\psi_{\beta}$, solving the Monge-Ampère equation 2.26, as a transcendental analog of $k^{-1} \log K_{k}(z, z)$, with $\beta$ playing the role of the degree $k$. Accordingly, the asymptotics in Theorem 2.12 can be viewed as a transcendental analog of the classical $k$ th root asymptotics for orthogonal polynomials (this point of view is developed from a complex geometric point of view in [21, where $k^{-1} \log K_{k}(z, z)$ appears as a Bergman metric). This interpretation is reinforced by the result in [27] showing that the condition that $(d V, \phi)$ is determining for $(K, \phi)$, appearing in Theorem 2.12 is equivalent to the following "transcendental" Bernstein-Markov-property of $(d V, \phi)$ : for any given $\epsilon>0$ there exists a constant $C>0$ such that

$$
\sup _{K} e^{\beta(\psi-\phi)} e \leq C e^{\epsilon \beta} \int e^{\beta(\psi-\phi)} d V
$$

for any $\psi \in \mathcal{L}\left(\mathbb{C}^{n}\right)$ and $\beta>0$ (which clearly implies the ordinary Bernstein-Markov-property 2.33).

Remark 2.17. When $d V$ is supported in $[0,1] \subset \mathbb{C}$ (and $\phi=0)$ the condition that $d V$ is determining coincides with Ulman's criterion for a measure $d V$ on $\mathbb{R}$ to have regular asymptotic distribution (discussed in [107, Chapter 4]).

2.6. Tropicalization at negative temperature, toric geometry and optimal transport. In complex geometry the process of tropicalization can sometimes to be invoked to replace an elusive complex geometric problem by a more tractable convex geometric one (see, for example, [75] for application to enumerative problems in algebraic geometry). In [29, 23] this philosophy is applied to the challenging negative temperature regime of the present setting, $\beta<0$ (discussed in the end of Section 2.3.2 above and in Section 7.4). The motivation comes from the Yau-Tian-Donaldson conjecture and, in particular, the construction of KählerEinstein metrics with positive Ricci curvature on toric varieties, as explained in 23 . Here we will focus on the connections to the present setting.

We first recall that, from an algebraic point of view, tropicalization amounts to replacing the ring $\mathbb{C}$ with the tropical semi-ring over $\mathbb{R}$, where addition of $a$ and $b$ is defined by $\max \{a, b\}$ and multiplication by ordinary addition. Accordingly, a polynomial $p(z)$ on $\mathbb{C}^{n}$ (or more, generally, a Laurent polynomial on $\mathbb{C}^{* n}$, is replaced by a piece-wise affine convex function $\phi(x)$ on $\mathbb{R}^{n}$ :

$$
p(z)=\sum_{m} c_{m} z^{m} \mapsto \max _{m} x \cdot m
$$

(up to an additive constant) where the max ranges over all $m \in \mathbb{Z}^{n}$ such that $c_{m} \neq 0$. Analytically, tropicalization can be viewed as a the limit of the complex geometric setting, 
where $\mathbb{C}^{* n}$ is collapsed onto $\mathbb{R}^{n}$ through the following map:

$$
\mathbb{C}^{* n} \rightarrow \mathbb{R}^{n}, \quad z \mapsto x:=\log (z):=\left(\log \left(\left|z_{1}\right|^{2}\right), \ldots, \log \left(\left|z_{n}\right|^{2}\right)\right) .
$$

Indeed, the association 2.36 amounts to replacing the plurisubharmonic functions $\psi(z)=$ $\log |p|^{2}$ on $\mathbb{C}^{* n}$ by the convex function $\psi_{\text {trop }}(x)$ on $\mathbb{R}^{n}$ defined by

$$
\psi_{\text {trop }}(x):=\lim _{c \rightarrow \infty} c^{-1} \psi\left(z^{m}\right) / 2
$$

Expanding the Vandermonde determinant as a sum over permutations $\sigma \in S_{N}$ reveals that the tropicalization of the attractive determinantal interaction energy $E_{a t t r}^{\left(N_{k}\right)}$ on $\mathbb{C}^{n N_{k}}$, appearing in formula 2.31, is the piece-wise affine convex function $E_{\text {trop }}^{\left(N_{k}\right)}$ on $\mathbb{R}^{n N_{k}}$ defined by

$$
E_{\text {trop }}^{\left(N_{k}\right)}\left(x_{1}, \ldots, x_{N_{k}}\right):=\frac{1}{N} \max \sum_{\sigma \in S_{N_{k}}} x_{1} \cdot p_{\sigma(1)}+\cdots+x_{N} \cdot p_{\sigma\left(N_{k}\right)},
$$

where $p_{1}, \ldots, p_{N}$ are are points of $P \cap\left(k^{-1} \mathbb{Z}\right)^{n}$, where $P$ denotes the convex polytope $P \Subset \mathbb{R}^{n}$ defined by the unit-simplex.

2.6.1. The setting of a convex body and toric varieties. Before proceeding to defining the corresponding tropicalization of the Gibbs measure defined by 2.22, it will be more natural to consider a more general class of Gibbs measures on $\left(\mathbb{C}^{* n}\right)^{N_{k}}$, associated to a given convex body $P$ in $\mathbb{R}^{n}$. Up to a harmless translation we may as well assume that 0 is contained in the interior of $P$. The setup in the previous sections may then be generalized by replacing the space $\mathcal{P}_{k}\left(\mathbb{C}^{n}\right)$ by the space $\mathcal{P}_{k P}\left(\mathbb{C}^{* n}\right)$ of Laurent polynomials on the complex torus $\mathbb{C}^{* n}$, spanned by all multinomials $z^{m}\left(=z_{1}^{m_{1}} \cdots z_{n}^{m_{n}}\right)$ with exponents $m \in k P \cap \mathbb{Z}^{n}$. Thus the dimension $N_{k}$ of $\mathcal{P}_{k P}\left(\mathbb{C}^{* n}\right)$ coincides with the number of integer points of the convex body $P$. Similarly, the Lelong class $\mathcal{L}\left(\mathbb{C}^{n}\right)$ is replaced by the space $\mathcal{L}_{P}\left(\mathbb{C}^{* n}\right)$ of all psh functions $\psi$ on $\mathbb{C}^{* n}$ satisfying the following growth condition:

$$
\psi_{P} \leq \log { }^{*} \phi_{P}+O(1), \quad \phi_{P}(x)=\sup _{p \in P} x \cdot p
$$

(the subspace $\mathcal{L}_{P,+}\left(\mathbb{C}^{* n}\right)$ is defined by requiring that equality holds above).

Remark 2.18. When $P$ is a rational polytope this more general setting corresponds to replacing $\mathbb{P}^{n}$ with a toric variety $X_{P}$ compactifying $\mathbb{C}^{* n}$ (compare Section 3.7 ). The case of a general convex body $P$ was introduced in [12] in the context of Bergman kernel asymptotics. See aso [7] for a systematic study of the class $\mathcal{L}_{P}\left(\mathbb{C}^{* n}\right)$ in connection to Fekete points.

Denoting by $d V_{\mathbb{C}^{* n}}$ the standard $\mathbb{C}^{* n}$-invariant volume form on $\mathbb{C}^{* n}$ it is tempting to try to define a probability measure on $\left(\mathbb{C}^{* n}\right)^{N_{k}}$ as the the normalization of the following measure on $\left(\mathbb{C}^{* n}\right)^{N_{k}}$

$$
\left|D^{\left(N_{k}\right)}\left(z_{1}, \ldots, z_{N_{k}}\right)\right|^{2 \beta / k} d V_{\mathbb{C}^{* n}}^{\otimes N}
$$

where $D^{\left(N_{k}\right)}$ now denotes the Vandermonde determinant associated to the space $\mathcal{P}_{k P}\left(\mathbb{C}^{* n}\right)$. Heuristically, i.e. assuming that the analog of Theorem 2.7 holds also for $\beta$ negative, one would expect that the large $N$-limit is described by solutions $\psi_{\beta}$ on $\mathbb{C}^{* n}$ to the equation

$$
M A(\psi)=e^{\beta \psi} d V_{\mathbb{C}^{* n}}^{\otimes N}
$$


Geometrically, this means that $\psi_{\beta}$ is the Kähler potential of a Kähler-Einstein metric $\omega_{\beta}$ with constant Ricci curvature:

$$
\operatorname{Ric} \omega_{\beta}=-\beta \omega_{\beta} .
$$

Since we have assumed that $\beta$ is negative this corresponds to constant positive Ricci curvature. Moreover, by a scaling argument one can assume that $\beta=-1$.

2.6.2. The tropical Gibbs measure. Unfortunately, the Vandermonde measure in formula 2.40 always has infinite mass, i.e. the corresponding normalization constant $Z_{N, \beta}$ is infinite, as follows from symmetry considerations. Indeed, the measure is invariant (modulo a multiplicative constant) under the diagonal action of $\mathbb{C}^{* n}$ on $\left(\mathbb{C}^{* n}\right)^{N_{k}}$. Since $\mathbb{C}^{* n}$ is non-compact this implies that $Z_{N, \beta}$ is infinite. This is a reflection of the fact that the group $\mathbb{C}^{* n}$ acts on the solution space to the Kähler-Einstein equation 2.41. The symmetry in question may be broken by turning to the weighted setting, i.e. by replacing $\left|D^{\left(N_{k}\right)}\right|$ with the weighted Vandermonde determinant $\left|D^{\left(N_{k}\right)}\right|_{k \phi}$, for a given continuous function $\phi(z)$ on $\mathbb{C}^{* n}$. We then consider the corresponding measure $\mu_{\beta}^{(N)}$ of the form 2.22, for an appropriate volume form $d V$ on $\mathbb{C}^{* n}$. Motivated by Kähler-Einstein geometry it is natural to take

$$
d V=e^{-\phi} d V_{\mathbb{C}^{* n}},
$$

and assume that $\phi \in \mathcal{L}_{P,+}\left(\mathbb{C}^{* n}\right)$. This ensures that one gets backs the Vandermonde measure, formula 2.40, when $\beta=-1$ and that $Z_{N, \beta}$ is finite for $\beta$ sufficiently close to 0 . Thus the weighted setting provides a regularization of the original divergent setting. The corresponding probability measure $\mu_{\beta}^{(N)}$ can, equivalently, be viewed as the Gibbs measure at unit-temperature associated to $N$ times the interaction energy

$$
E_{\beta}^{\left(N_{k}\right)}\left(z_{1}, \ldots z_{N_{k}}\right):=-\beta E_{\text {attr }}^{\left(N_{k}\right)}\left(z_{1}, \ldots z_{N}\right)+(1+\beta) \sum_{i=1}^{N_{k}} \phi\left(z_{i}\right)
$$

and volume form $d V_{\mathbb{C}^{* n}}^{\otimes N}$, i.e.

$$
\mu_{\beta}^{(N)}=\frac{1}{Z_{N, \beta}} e^{-N E_{\beta}^{\left(N_{k}\right)}} d V_{\mathbb{C}^{* n}}^{\otimes N}
$$

In view of formula 2.38 and 2.39, the tropicalization of $E_{\gamma}^{\left(N_{k}\right)}$ is given by

$$
E_{\beta, \text { trop }}^{\left(N_{k}\right)}\left(x_{1}, \ldots x_{N_{k}}\right):=-\beta E_{\text {trop }}^{\left(N_{k}\right)}\left(x_{1}, \ldots, x_{N_{k}}\right)+(1+\beta) \sum_{i=1}^{N_{k}} \phi_{P}\left(x_{i}\right)
$$

Finally, one is thus led to define the tropicalization of the Gibbs measure $\mu_{\beta}^{(N)}$ on $\left(\mathbb{C}^{* n}\right)^{N_{k}}$, associated to a given convex body $P$, as the following Gibbs measure on $\left(\mathbb{R}^{n}\right)^{N_{k}}$ :

$$
\mu_{\beta, \text { trop }}^{(N)}:=\frac{1}{Z_{N_{k}, \beta}} e^{-N E_{\beta, t r o p}^{\left(N_{k}\right)}} d x^{\otimes N_{k}},
$$

The following result [23] shows that the convergence in Theorem 2.7 can be extended to the negative temperature regime, down to a critical negative inverse temperature $\beta_{c}\left(=-R_{P}\right)$, if the original complex problem is replaced by its tropical analogue. [See also the ArXiv version of [29], where the proof of the result originally appeared]. 
Theorem 2.19. Let $P$ be a convex body in $\mathbb{R}^{n}$ containing 0 in its interior. Then,

- For $k$ sufficiently large, the corresponding Gibbs measure $\mu_{\beta, t r o p}^{\left(N_{k}\right)}$ is a well-defined probability measure, i.e. $Z_{N_{k}, \beta}<\infty$ iff $\beta<-R_{P}$, where $\left.\left.R_{P} \in\right] 0,1\right]$ is the following invariant of $P$ :

$$
R_{P}:=\frac{\|q\|}{\left\|q-b_{P}\right\|}
$$

where $q$ is the point in $\partial P$ where the line segment starting at the barycenter $b_{P}$ of $P$ and passing through 0 meets $\partial P$.

- If $\beta>-R_{P}$, then the random measure $\delta_{N_{k}}$ on $\left(\left(\mathbb{R}^{n}\right)^{N_{k}}, \mu_{\beta, t r o p}^{\left(N_{k}\right)}\right)$ converges in law, as $N \rightarrow \infty$, towards $\mu_{\beta}=e^{\beta\left(u_{\beta}-\phi_{P}\right)} e^{-\phi_{p}} d x$, where $u_{\beta}(x)$ is the unique convex solution to the following real Monge-Ampère equation on $\mathbb{R}^{n}$

$$
\operatorname{det}\left(\nabla^{2} u\right)=e^{\beta\left(u-\phi_{P}\right)} e^{-\phi_{p}} d x
$$

subject to the "second boundary condition"

$$
\overline{(\nabla u)\left(\mathbb{R}^{n}\right)}=P
$$

- If $R_{P}=1$, i.e. if 0 is the barycenter of $P$, then $u_{\beta}$ converges uniformly on $\mathbb{R}^{n}$, as $\beta \rightarrow-1$, to a smooth solution $u_{1}$ of the equation

$$
\operatorname{det}\left(\nabla^{2} u\right)=e^{-u}
$$

subject to 2.46 .

The pull-back $\psi$ to $\mathbb{C}^{* n}$, under the map Log of any solution $u$ of the equation 2.47 solves the Kähler-Einstein equation 2.41 and is in $\mathcal{L}_{P}\left(\mathbb{C}^{* n}\right)$. Moreover, when $P$ is a rational polytope, the last property ensures that $\omega$ extends to define a Kähler-Einstein metric on the toric (log) Fano variety $X_{P}$ compactifying $\mathbb{C}^{* n}$. By the toric case of the Yau-Tian-Donaldson conjecture $X_{P}$ admits a Kähler-Einstein metric iff $b_{P}=0$. Thus Theorem 2.19 provides a probabilistic construction of Kähler-Einstein metrics on toric Fano varieties, when such metrics exist.

For a general convex body $P$ it follows from 25 that the equation 2.47 admits a solution iff $b_{P}=0$. Moreover, the solution is unique modulo the action of $\mathbb{R}^{n}$ by translations. In fact, the previous theorem holds more generally when $\phi_{P}$ is replaced by a given convex weight function $\phi$ such that $\phi=\phi_{P}+O(1)$. When $b_{p}=0$ the corresponding limit of $u_{\beta}$, as $\beta \rightarrow-1$, singles out a particular solution of the equation 2.47, depending on the choice of weight function $\phi$. From the point of view of statistical mechanics this can be interpreted as an instance of spontaneous symmetry breaking (see the appendix). Moreover, the critical inverse temperature $-R_{P}$ is a tropical (and higher dimensional analog) of the critical negative temperature of the Coulomb gas in $\mathbb{C}$, exhibited in [77, 42].

2.6.3. Optimal transport formulation. The tropicalized setting may be formulated in terms of optimal transport theory. Indeed, setting

$$
\mu_{0}=N^{-1} \sum_{i=1}^{N} \delta_{x_{i}}, \quad \mu_{1}:=N^{-1} \sum_{i=1}^{N} \delta_{p_{i}},
$$

the tropicalization $E_{\text {trop }}^{(N)}\left(x_{1}, \ldots, x_{N}\right)$ of the attractive determinantal interaction energy $E_{\text {attr }}\left(z_{1}, \ldots, z_{N}\right)$ (formula 2.6.3) coincides with minus the minimal cost $C\left(\mu_{0}, \mu_{1}\right)$ of transportation between 
the probability measures $\mu_{0}$ and $\mu_{1}$, with respect to the standard quadratic cost function $c(x, y):=-x \cdot y:$

$$
C\left(\mu_{0}, \mu_{1}\right):=\inf _{\gamma \in \Pi\left(\mu_{0}, \mu_{1}\right)} C[\gamma] C[\gamma]:=\int c \gamma
$$

where the minimum runs over all transport plans $\gamma$, i.e. all probability measures on $\mathbb{R}^{n} \times \mathbb{R}^{n}$ with marginals $\mu_{0}$ and $\mu_{1}$, respectively. It follows readily from this interpretation of $E_{\text {trop }}^{(N)}$ that, denoting by $\nu_{P}$ the uniform probability measure on $P$,

$$
N^{-1} \sum_{i=1}^{N} \delta_{x_{i}} \rightarrow \mu \Longrightarrow-E_{\text {trop }}^{(N)}\left(x_{1}, \ldots x_{N}\right) \rightarrow C\left(\mu, \nu_{P}\right),
$$

if the convergence towards $\mu$ holds weakly and moreover the first moments converge. The convergence towards $C\left(\mu, \nu_{P}\right)$ is a tropical analog of the Gamma-convergence of $-E_{\text {attr }}^{(N)}\left(z_{1}, \ldots, z_{N}\right)$ towards $E(\mu)$ (formula 2.15). In fact, when $\mu$ is invariant under the torus $T^{n}$ the minimal cost $C\left(\mu, \nu_{P}\right)$ coincides with the (non-weighted) pluricomplex energy $E(\mu)$, as observed in [17] (see also [25]). This is a reflection of the, essentially well-known fact [22], that a convex function $u$ on $\mathbb{R}^{n}$ solves the real Monge-Ampère equation

$$
\operatorname{det}\left(\nabla^{2} u\right)=\mu
$$

(which is the infinite temperature case, $\beta=0$, of the equation 2.45) iff the corresponding gradient map

$$
T: x \mapsto \nabla u(x)
$$

is the optimal map $T$ transporting $\mu$ to the uniform measure $\nu_{P}$ on the convex body $P$. This means that $\gamma:=(I \times T) * \mu$ realizes the infimum in formula 2.48 ,

2.7. Further references and developments. Before zooming in on the details in the account above, let us mention some other related developments.

2.7.1. Fekete points. The rate of convergence for the Fekete points, as $N \rightarrow \infty$ has been made quantitative in [81, 51. For example, in the case when $(X, L)$ is a polarized manifold and $L$ is endowed with a smooth and strictly positively curved metric, the following sharp rate is established in [81] when $K=X$ :

$$
d_{1}\left(\delta_{N}, \mu_{K}\right) \sim k^{-1 / 2}
$$

where $d_{1}$ denotes the Wasserstein $L^{1}$-distance on $\mathcal{M}_{1}(X)$. Generalizations to less regular metrics and with $K$ an appropriate proper compact subset are given in [51]. Recently, the problem of numerical simulation of approximate Fekete points has been studied; see [36] and references therein.

2.7.2. Large deviations for Vandermonde determinants. In the case $\beta=\infty$ asymptotics of integrals of Vandermonde determinants and large deviations in $\mathbb{C}^{n}$ have also been obtained in [33]. The relevance of the Bernstein-Markov condition on the reference measure $d V$ in this context originates in [32]. In the case of a polarized manifold $(X, L)$ quantitative rates of convergences for the corresponding empirical measures have been obtained in [81, 52]. For example, as shown in [81], when $(X, L)$ is a polarized manifold and $L$ is endowed with a smooth and strictly positively curved metric a rate as in formula 2.49 holds when $d_{1}\left(\delta_{N}, \mu_{K}\right)$ is replaced 
by the corresponding expectations $\mathbb{E} d_{1}\left(\delta_{N}, \mu_{K}\right)$. Similar results hold if a quantitative version of the Bernstein-Markov property is assumed [52] (which, for example, is satisfied if $K$ is a smooth totally real submanifold). It would be interesting establish quantitative rates in the case when $\beta<\infty$. In another direction, in the case when $(X, L)$ is an abelian variety (i.e $X$ is a compact complex torus) a real analog of Theorem [5.7, which holds also for negative $\beta$, has been established in [74].

2.7.3. The limits $\beta \rightarrow 0$ and $\beta \rightarrow \infty$. When $d V$ is a continuous volume form, parametrized complex Monge-Ampère equations of the form 6.1 have previously been used in 64 to give a new proof of the existence of a continuous solutions of the corresponding Calabi-Yau equation. Briefly, the solution $\varphi_{\beta}$ for $\beta>0$ is first obtained as the sup of viscosity subsolutions. Then it is shown that $\varphi_{\beta}$ converges to a solution of the Calabi-Yau equation, when $\beta \rightarrow 0$. In the present setting the role of viscosity subsolutions is, loosely speaking, played by the explicit Kähler potentials in Corollary 2.10, This probabilistic approach thus appears to be very different from the viscosity approach 2 Incidentally, as shown in [70], the method of regularizing psh envelopes $P u$ by solutions $\varphi_{\beta}$ in the opposite "zero-temperature limit" $\beta \rightarrow \infty$ (Theorems [2.12, 6.1) can be put to use in the viscosity theory of [70]. For example, a key result in [70] says that if $u_{1}$ and $u_{2}$ are two super solutions, then so is the psh-envelope $P\left(\min \left\{u_{1}, u_{2}\right\}\right)$. This is shown by generalizing the convergence of $\varphi_{\beta}$ in Theorem 6.1 to a very irregular setting where $u$ is merely assumed to a bounded Borel function and $d V$ a general measure not charging pluripolar subsets. The method of regularizing envelopes by solutions of Monge-Ampère type equations has also been extended and applied in various other directions [88, 50, 108, 46]. As observed in [97 the equation 5.13 can also be obtained from the Ricci flow via a backwards Euler discretization. Accordingly, the corresponding continuity path is called the Ricci continuity path in [76], where it (or rather its "conical" generalization) plays a crucial role in the construction of Kähler-Einstein metrics with edge/cone singularities, by deforming the trivial solution at $\beta=\infty$ (for $\varphi_{\infty}$ a given Kähler potential) to a Kähler-Einstein metric at $\beta= \pm 1$.

\section{Complex Geometric Setup}

In this section we provide some background from complex geometry and pluripotential theory (see the books [49, 69] for further general background).

3.1. The local setting. Setting $z:=x+i y \in \mathbb{C}^{n}$ the space $\Omega^{1}\left(\mathbb{C}^{n}\right)$ of all complex one-forms on $\mathbb{C}^{n}$ decomposes as a sum

$$
\Omega^{1}\left(\mathbb{C}^{n}\right)=\Omega^{1,0}\left(\mathbb{C}^{n}\right)+\Omega^{0,1}\left(\mathbb{C}^{n}\right),
$$

of the two subspaces spanned by $\left\{d z_{i}\right\}$ and $\left\{d \bar{z}_{i}\right\}$, respectively. This induces a decomposition of the exterior algebra of all complex differential forms $\Omega\left(\mathbb{C}^{n}\right)$ into forms of bidegree $(p, q)$, where $p \leq n$ and $q \leq n$. Accordingly, the exterior derivative $d$ decomposes as $d=\partial+\bar{\partial}$, where

$$
\partial \phi:=\sum_{i=1}^{n} \frac{\partial \phi}{\partial z_{i}} d z_{i}, \frac{\partial}{\partial z_{i}}:=\left(\frac{\partial}{\partial x_{i}}-i \frac{\partial}{\partial y_{i}}\right) / 2,
$$

\footnotetext{
${ }^{2}$ On the other hand, it is somewhat reminiscent of the "vanishing viscosity method" for constructing viscosity solutions $v$ to a PDE as a limit $v_{\epsilon}$ of equations obtained by adding a regularizing diffusion term, proportional to a small "viscosity parameter" $\epsilon$ (whose role here is played by $N_{k}^{-1}$ ).
} 
and taking its complex conjugate defines the $(0,1)$-form $\bar{\partial} \phi$. In particular,

$$
\omega^{\phi}:=\frac{i}{2 \pi} \partial \bar{\partial} \phi=\frac{i}{2 \pi} \sum_{i, j \leq n} \frac{\partial^{2} \phi}{\partial z_{i} \partial \bar{z}_{j}} d z_{i} \wedge d \bar{z}_{j},
$$

defines a real $(1,1)$-form (the normalization above turns out to be convenient). Such a smooth form $\omega$ is said to be positive (Kähler), written as $\omega \geq 0(\omega>0)$, if the corresponding Hermitian matrix is semi-positive (positive definite) at any point.

If $\phi$ is smooth, then $\phi$ is said to be plurisubharmonic (psh) if $\omega^{\phi} \geq 0$. A general function $\phi \in L_{l o c}^{1}$ is said to be psh if it is strongly upper semi-continuous and $\omega^{\phi} \geq 0$ holds in the weak sense of currents. More generally, given a real smooth $(1,1)$-form $\omega$ a function $\varphi$ is said to be $\omega-p s h$ if

$$
\omega_{\varphi}:=\omega+\frac{i}{2 \pi} \partial \bar{\partial} \varphi \geq 0
$$

If $\omega:=\omega^{\phi_{0}}$ this means that $\phi$ is psh iff $\varphi:=\phi-\phi_{0}$ is $\omega-$ psh.

For $\phi$ psh and smooth the Monge-Ampère measure is the measure defined by

$$
M A(\phi):=\left(\omega^{\phi}\right)^{n}
$$

in terms of exterior products. The extension to locally bounded psh $\phi$ was introduced by Bedford-Taylor. The extension may be characterized by the property that it is continuous under decreasing limits of psh locally bounded functions $\phi_{j}$.

3.2. Polarized compact manifolds $(X, L)$ and metrics on $L$. The local complex analytic notions above naturally extend to the global setting where $\mathbb{C}^{n}$ is replaced by a complex manifold (using that the decomposition 3.1 is invariant under a holomorphic change of coordinates). However, if $X$ is compact, then all psh functions $\phi$ on $X$ are constant (by the maximum principle). Instead the role of a (say, smooth) psh function $\phi$ on $\mathbb{C}^{n}$ is played by a positively curved metric on a line bundle $L \rightarrow X$ (using additive notations for metrics). To briefly explain this standard complex geometric framework, first recall that a line bundle $L$ over a complex manifold $X$ is, by definition, a complex manifold (called the total space of $L$ ) with a surjective holomorphic map

$$
\pi: L \rightarrow X
$$

such that the fibers $L_{x}$ of $\pi$ are one-dimensional complex vector spaces and such that $\pi$ is locally trivial. In other words, any point $x \in X$ admits a neighborhood $U$ and an isomorphism (i.e. a $\mathbb{C}^{*}$-equivariant biholomorphic map) between $L_{\mid U}$ and $U \times \mathbb{C}$, making the following diagram commutative:

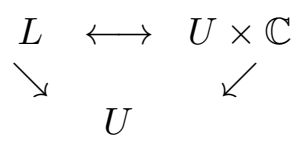

Fixing such an isomorphism holomorphic sections of $L \rightarrow U$ may be identified with holomorphic functions on $U$. In particular, the function 1 over $U$ corresponds to a non-vanishing holomorphic section $e_{U}$ of $L \rightarrow U$. Now, a smooth (Hermitian) metric $\|\cdot\|$ on the line bundle $L$ is, by definition, a smooth family of Hermitian metrics on the one-dimensional complex subspaces $L_{x}$. Given a covering $U_{i}$ of $X$ and trivializations $e_{U_{i}}$ of $L \rightarrow U_{i}$ a metric $\|\cdot\|$ on $L$ may be represented by the following family of local functions $\phi_{U_{i}}$ on $U_{i}$ :

$$
\left\|e_{U_{i}}\right\|^{2}=e^{-\phi_{U_{i}}}
$$


(accordingly a metric on $L$ is often, in additive notation, denoted by the symbol $\phi$, which is called a weight). The functions $\phi_{U_{i}}$ do not agree on overlaps, in general. But the (normalized) curvature form $\omega$ of the metric $\|\cdot\|$ is a well-defined closed two-form on $X$, locally defined by

$$
\omega_{\mid U_{i}}:=\omega^{\phi_{U_{i}}}
$$

Example 3.1. If $X$ is a complex manifold then the trivial line bundle $L_{0} \rightarrow X$ is $X \times \mathbb{C}$ with its natural projection to $X$. Thus the function 1 on $X$ defines a global holomorphic trivialization of $L_{0}$. The trivial metric $\|\cdot\|$ on $L_{0}$ is defined by $\|1\|:=1$, i.e. the corresponding weight function $\phi$ vanishes identically on $X$. Accordingly, so does its curvature form.

Definition 3.2. A polarized manifold $(X, L)$ is a compact complex manifold $X$ endowed with a positive line bundle, i.e. a line bundle $L$ which admits some smooth metric with strictly positive curvature form.

According to Kodaira's embedding theorem any polarized manifold $X$ is projective, i.e. it may be realized as a complex (algebraic) submanifold of projective space (see Remark 3.12).

Singular metrics on $L$ may be defined in a similar way. In particular, a singular metric is said to have positive curvature if the local weights $\phi_{U_{i}}$ are psh, i.e. the corresponding curvature form $\omega$ defines a positive $(1,1)$-current on $X$. The difference between the weights of two different metrics is always a globally well-defined function on $X$ (as a consequence, the curvature currents of any two metrics on $L$ are cohomologous and represent the first Chern class $\left.c_{1}(L) \in H^{2}(X, \mathbb{Z})\right)$.

Example 3.3. The (normalized) Ricci curvature of a Kähler metric $\omega$ on a complex manifold $X$ (identified with a Riemannian metric) may be expressed as

$$
\operatorname{Ric} \omega=-\frac{i}{2 \pi} \partial \bar{\partial} \log \left(\omega^{n} / d \lambda\right),
$$

where $d \lambda$ denotes Lebesgue measure, expressed in terms of local holomorphic coordinates $z_{1}, \ldots, z_{n}$. Denoting by $K_{X}$ the canonical line bundle of $X$, i.e. the top exterior power of the holomorphic cotangent bundle $T^{*} X$, this means that $-\operatorname{Ric} \omega$ coincides with the curvature of the metric $\|\cdot\|$ on the line bundle $K_{X}$ induced by the volume form $\omega^{n}$. Indeed, $e:=d z_{1} \wedge \ldots \wedge d z_{n}$ is a local trivialization of $K_{X}$ and $\|e\|^{2}:=c_{n} \omega^{n} / e \wedge \bar{e}$, for a suitable constant $c_{n}$. By formula 3.4. if $\psi_{\beta}$ is a smooth psh solution of the Monge-Ampère equation 2.26, then the corresponding Kähler metric $\omega_{\beta}\left(:=\omega^{\psi_{\beta}}\right)$ satisfies the twisted Kähler-Einstein equation 2.30 with

$$
\tau:=\beta \frac{i}{2 \pi} \partial \bar{\partial} \phi-\frac{i}{2 \pi} \partial \bar{\partial} \log \frac{d V}{d \lambda}
$$

(note that the last term vanishes if $d V$ is equal to Lebesgue measure $d \lambda$ ).

Remark 3.4. Given a metric $\|\cdot\|$ on $L$ we will use the same notation $\|\cdot\|$ for the induced metric on the tensor products of $L$ over $X$, obtained by imposing that $\|\cdot\|$ be multiplicative. In particular, if $\phi$ is a local weight for $\|\cdot\|$ (defined wrt the local trivialization $s_{U}$ ) then $k \phi$ is a local weight for the $k$ th tensor product of $L$ (defined wrt the local trivialization $s_{U}^{\otimes k}$ ). This motivates using the additive notation $k L$ for tensor products. More generally, we will use the same notation $\|\cdot\|$ for the induced metrics on the line bundles $(k L)^{\bigotimes N}$ over the $N$-fold products $X^{N}$. 
3.2.1. Holomorphic sections of $L$. The complex vector space of all global holomorphic sections of $L \rightarrow X$ will be denoted by $H^{0}(X, L)$. The line bundle $L$ is said to be big if there exists a positive number $V$ such that

$$
N_{k}:=H^{0}(X, k L)=\frac{V}{n !} k^{n}+o\left(k^{n}\right),
$$

using additive notation $k L$ for tensor products of line bundles. In particular, any positive line bundle $L$ is big (by the Hilbert-Samuel theorem). The results in [27, 19] apply to general big line bundles, but here we will only consider the simpler case when $L$ is positive, i.e. the case of a polarized manifold $(X, L)$ ( as in [15, 18]).

There is natural correspondence between the space $H^{0}(X, L)$ and the space of all 1-homogeneous holomorphic functions on the complex manifold defined by the total space of the dual line bundle $L^{*} \rightarrow X$, endowed with its standard $\mathbb{C}^{*}$-action. Indeed, if $s_{k} \in H^{0}(X, k L)$ and $w \in L^{*}$. Then

$$
S_{k}(w):=\left\langle s_{k}, w^{\otimes k}\right\rangle
$$

is a well-defined $k$-homogeneous function on $L^{*}$ (since $s_{k}$ takes values in the $k$ th tensor power of $L)$.

Similarly, the space of metrics $\|\cdot\|$ on $L$ may be identified with the space of all positively 1-homogeneous non-negative functions $N$ on the total space of $L^{*} \rightarrow X$. The curvature form of $\|\cdot\|$ is (strictly) positive on $X$ iff $N^{2}$ is (strictly) psh on $L^{*}-\{0\}$. Indeed, fixing local holomorphic coordinates $z$ on $X$ and a local trivialization of $L$ induces local holomorphic coordinates $(z, w)$ on $L^{*}$ such that

$$
N^{2}(z, w)=|w|^{2} e^{\phi(z)}
$$

3.3. $\omega$-psh functions on Kähler manifolds. Let $(X, L)$ be a polarized manifold. Fixing a reference metric $\|\cdot\|_{0}$ and setting

$$
\|\cdot\|_{\varphi}:=\|\cdot\|_{0} e^{-\varphi / 2}
$$

the space all metrics $\|\cdot\|$ on $L$ with positive curvature current may be identified with the space $\operatorname{PSH}(X, \omega)$ of all $\omega$-psh functions $\varphi$ on $X$. The correspondence is made so that $\omega_{\varphi}$ (formula 3.3) is the normalized curvature of the corresponding metric $\|\cdot\|_{\varphi}$ on $L$.

Example 3.5. If $s \in H^{0}(X, k L)$ and $\|\cdot\|$ is a metric on $L$ with curvature form $\omega$, then the function $k^{-1} \log \|s\|^{2}$ is in $P S H(X, \omega)$, since $\log |s(z)|^{2}$ defines a singular metric on $L$ with positive curvature current. Concretely, locally representing $s=s_{U}(z) e_{U}$ over $U \subset X$, where $e_{U}$ is a trivializing holomorphic section of $L \rightarrow U$ and $s_{U}(z)$ is a local holomorphic function, we have that $\log \left|s_{U}(z)\right|^{2}$ is psh and hence $\log \|s\|^{2}=\log \left(\left|s_{U}(z)\right|^{2} e^{-k \phi_{U}}\right)$ is $k \omega-$ psh.

Given a real closed $(1,1)$ - form $\omega$ the space $P S H(X, \omega)$ can be defined without any reference to line bundles. In fact, $\omega$ arises as the normalized curvature form of a metric on a line bundle over $X$ iff $\omega$ has integral periods (i.e. it defines a class in the De Rham cohomology $H^{2}(X, \mathbb{Z})$ ). Accordingly, from an analytic point of view it is more naturally to consider the more general setting of a of a pair $(X, \omega)$ consisting of a compact complex manifold $X$ and real closed $(1,1)-$ form $\omega$, with continuous potentials on $X$, which is cohomologous to a Kähler form. In other words,

$$
\omega=\omega_{0}+\frac{i}{2 \pi} \partial \bar{\partial} u
$$


for some Kähler form $\omega_{0}$ on $X$ and continuous function $u$ on $X$.

We recall the following fundamental properties of the space $P S H(X, \omega)[68$.

Proposition 3.6. Assume that $X$ is compact and that $\omega$ is cohomologous to a Kähler form.

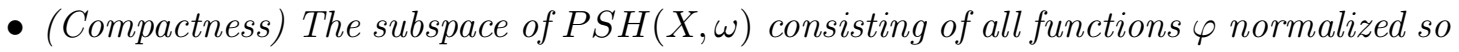
that either $\int_{X} \varphi d V=0$ (for a given volume form $d V$ on $X$ ) or $\sup _{X} \varphi=0$ is compact with respect to the $L^{1}$-topology.

- (Approximation) Any element $\varphi \in P S H(X, \omega)$ can be written as a decreasing limit of Kähler potentials $\varphi_{j}$, i.e. smooth functions in $P H S(X, \omega)$ such that $\omega_{\varphi}$ is a Kähler form (i.e. $\left.\omega_{\varphi}>0\right)$.

Proof. The compactness follows from embedding $P S H(X, \omega)$ into the compact space $\mathcal{M}_{1}(X)$ (by mapping $\varphi$ to the Laplace type measure $\omega_{\varphi} \wedge \tau^{n-1}$, for a fixed Kähler form $\tau$ on $X$ ). As for the approximation result it is a special case of Demailly's general approximation results for positive currents. Interestingly, the "zero-temperature" limit $\beta \rightarrow \infty$ studied in Section 6 provides an alternative "PDE-proof" of Demailly's approximation result for $P S H(X, \omega)$. To see this first take a sequence $u_{j} \in C^{\infty}(X)$ decreasing to $\varphi$. By monotonicity, $P_{\omega}\left(u_{j}\right)$ decreases $\varphi$ and hence it is enough to verify that $P_{\omega}(u)$ can be uniformly approximated by Kähler potentials when $u$ i smooth. But, as shown in [21], $P_{\omega}(u)$ is the uniform limit of the functions $\varphi_{\beta}$ solving the corresponding Monge-Ampère equation 6.1 (for a fixed volume form $d V$ ) and $\varphi_{\beta}$ is smooth by [5, 110]. As discussed in [21] this regularization scheme can by viewed as a transcendental analog of the well-known Bergman kernel approach to regularization used in case of a polarized manifold $(X, L)$.

3.4. The non-pluripolar Monge-Ampère measure and the functional $\mathcal{E}(\varphi)$. Let $(X, L)$ be a polarized compact manifold and fix a smooth metric $\|\cdot\|$ on $L$ with curvature form $\omega$. Then we can, as explained above, identify (singular) positively curved metrics $\phi$ on $L$ with $\omega$-psh functions $\varphi$ on $L$. For a smooth $\omega$-psh function the (normalized) Monge-Ampère measure of $\varphi$ (with respect to $\omega$ ) is defined as

$$
M A_{\omega}(\varphi):=\omega_{\varphi}^{n} / V
$$

i.e. as the top exterior power of the corresponding curvature form divided by the volume $V$ of the class $[\omega]$. More generally, by the local pluripotential theory of Bedford-Taylor, the expression in formula 3.6 makes locally sense for any bounded $\omega$-psh function and defines a probability measure on $X$ (which does not put charge on pluripolar subsets, i.e. sets which are locally contained in the $-\infty$-locus of a psh function). The corresponding Monge-Ampère measure $M A$ is continuous with respect to decreasing sequences of bounded $\theta$-psh functions. In general, following [10], for any $\varphi \in P S H(X, \theta)$ we will denote by $M A_{\omega}(\varphi)$ the non-pluripolar Monge-Ampère measure, which is a globally well-defined measure on $X$ not charging pluripolar subsets. The measure $M A_{\omega}(\varphi)$ is defined by replacing the ordinary wedge products with the so called non-pluripolar products introduced in [10]) and satisfies

$$
\int_{X} M A_{\omega}(\varphi) \leq 1
$$

The Monge-Ampère measure $M A_{\omega}$, viewed as a one-form on the space $P S H(X, \omega) \cap L^{\infty}$ is exact and we shall denote by $\mathcal{E}$ the corresponding primitive. In other words,

$$
d \mathcal{E}_{\mid \varphi}=M A_{\omega}(\varphi)
$$


(in the sense that $d \mathcal{E}(\varphi+t u) / d t=\int_{X} M A(\varphi) u / V$ at $\left.t=0\right)$. The functional $\mathcal{E}$ on $P S H(X, \omega) \cap$ $L^{\infty}$ is only defined up to an additive constant that we shall fix by imposing the normalization condition

$$
\mathcal{E}(\varphi(X, \omega))=0
$$

where $\varphi_{(X, \omega)}=0$ when $\omega \geq 0$ and in general it is defined as a global extremal function in the section below (formula 3.11). Occasionally, we we will use the notation $\mathcal{E}_{\omega}$ to indicate the dependence on $\omega$ in the definition of the functional $\mathcal{E}$. Integrating the defining relation 3.7 along a line segment in $P S H(X, \omega) \cap L^{\infty}$ reveals that

$$
\mathcal{E}(\varphi)=\frac{1}{(n+1) V} \int_{X} \varphi \sum_{j=0}^{n} \omega^{j} \wedge \omega_{\varphi}^{n-j}
$$

in the case when $\omega \geq 0$ (but the explicit formula for $\mathcal{E}$ will not really be used in the sequel). We will also denote by $\mathcal{E}$ the smallest upper semi-continuous extension of $\mathcal{E}$ to all of $P S H(X, \omega)$, introduced in [28], and write

$$
\mathcal{E}^{1}(X, \omega):=\{\varphi \in \operatorname{PSH}(X, \omega): \mathcal{E}(\varphi)>-\infty\},
$$

which is called the space of all functions on $X$ with finite energy. 3 The Monge-Ampère measure of any $\varphi \in \mathcal{E}^{1}(X, \omega)$ defines a probability measure on $X$.

3.5. The PSH-projection $P_{\omega}$ and the global Siciak extremal function. Next, we recall the definition of the operator $P_{\omega}$ introduced in [26]. Given $u \in C(X)$ we set

$$
\left(P_{\omega} u\right)(x):=\sup _{P S H(X, \omega)}\{\varphi(x): \varphi \leq u \text { on } X\}
$$

which defines an operator

$$
P_{\omega}: C(X) \rightarrow P S H\left(X, \omega_{0}\right)
$$

from $C^{0}(X)$ to the space $P S H\left(X, \omega_{0}\right)$ of all $\omega$-psh functions on $X$, i.e. all upper semicontinuous functions $\varphi$ in $L^{1}(X)$ such that $\omega_{\varphi} \geq 0$ in the sense of currents.

Now the global Siciak extremal function of $(X, \omega)$ may be defined by

$$
\varphi_{(X, \omega)}:=P_{\omega}(0) .
$$

More generally, if $K$ is a locally non-pluripolar subset of $X$, then the global Siciak extremal function of $(K, \omega)$ is defined as the upper upper-semicontinuous regularization of $P_{(K, \omega)}(0)$, where $P_{(K, \omega)}(u)$ is defined as in formula 3.10, but only demanding that $\varphi \leq u$ on $K$. The assumption on $K$ ensures that $P_{(K, \omega)}(u)$ is a well-defined finite function and hence $\omega$-psh (see [68] where the global Kähler analogs of Siciak's extremal function in $\mathbb{C}^{n}$ were first introduced).

Remark 3.7. Since we have assumed that $(X, L)$ is polarized (or more generally, that $\omega$ is cohomologous to a Kähler form) the operator $P_{\omega}$ preserves $C(X)$ (as follows from Prop 3.6). Hence, it defines a (non-linear) projection operator from $C^{0}(X)$ onto $P S H(X, \omega) \cap C^{0}(X)$. Anyway, for the present purposes it is enough to observe that $P_{\omega}$ preserves $L^{\infty}$, as follows directly from the definition.

The following important "orthogonality relation" holds for the projection operator $P_{\omega}$ :

$$
\int_{X}\left(u-P_{\omega} u\right) M A_{\omega}\left(P_{\omega} u\right)=0
$$

\footnotetext{
${ }^{3}$ We will adopt the notation from [15], which is different from the one in 28 .
} 
Indeed, since $P_{\omega} u \leq u$, this is equivalent to saying that $P_{\omega} u=u$ almost everywhere wrt the measure $M A_{\omega}\left(P_{\omega} u\right)$, which in turn follows from a "balayage"/maximum principle type argument (see [26]).

3.6. The pluricomplex energy $E_{\omega}(\mu)$ on polarized manifolds. Following [28] the pluricomplex energy $E_{\omega}(\mu)$ of a probability measure $\mu$ is defined by

$$
E_{\omega}(\mu):=\sup _{\varphi \in \mathcal{E}^{1}(X, \omega)} \mathcal{E}(\varphi)-\langle\varphi, \mu\rangle,
$$

which is automatically lsc and convex on $\mathcal{M}_{1}(X)$ (by the approximation property in Prop 3.6 the sup may equivalently be taken over $\operatorname{PSH}(X, \omega) \cap C(X)$ or $\left.P S H(X, \omega) \cap C^{\infty}(X)\right)$.

As recalled in the following theorem the sup defining $E_{\omega}$ is in fact attained, if $E_{\omega}(\mu)<\infty$ :

$$
E_{\omega}(\mu):=\mathcal{E}\left(\varphi_{\mu}\right)-\left\langle\varphi_{\mu}, \mu\right\rangle
$$

for a unique function $\varphi_{\mu} \in \mathcal{E}^{1}(X, \theta) / \mathbb{R}$. Moreover

$$
M A\left(\varphi_{\mu}\right)=\mu \text {. }
$$

Theorem 3.8. 28] The following is equivalent for a probability measure $\mu$ on $X$ :

- $E_{\omega}(\mu)<\infty$

- $\langle\varphi, \mu\rangle<\infty$ for all $\varphi \in \mathcal{E}^{1}(X, \omega)$

- $\mu$ has a potential $\varphi_{\mu} \in \mathcal{E}^{1}(X, \omega)$, i.e. equation 3.15 holds

Moreover, $\varphi_{\mu}$ is uniquely determined $\bmod \mathbb{R}$, i.e. up to an additive constant and can be characterized as the function maximizing the functional whose sup defines $E_{\omega}(\mu)$ (formula 3.13).

In particular, if $\mu$ charges a pluripolar subset then $E_{\omega}(\mu)=\infty$. The previous theorem implies the following result [15, Prop 2.7]:

Proposition 3.9. Assume that $\mu \in \mathcal{M}_{1}(X)$ has finite energy, i.e. $E_{\omega}(\mu)<\infty$. Then $-\varphi_{\mu}$ is a sub-gradient for the functional $E_{\omega}$ at $\mu$, i.e.

$$
E_{\omega}(\nu) \geq E_{\omega}(\mu)-\left\langle\nu-\mu, \varphi_{\mu}\right\rangle
$$

for any $\nu \in \mathcal{M}_{1}(X)$. As a consequence, if $\mu_{t}$ is an affine curve in $\mathcal{M}_{1}(X)$, defined for $\left.t \in\right]-\epsilon, \epsilon[$ and such that $\mu_{0}=\mu$, then

$$
{\frac{d E_{\omega}\left(\mu_{t}\right)}{d t}}_{\mid t=0}=-\left\langle\mu_{1}-\mu, \varphi_{\mu}\right\rangle
$$

Remark 3.10. By the previous proposition the differential of the restriction of the functional $E_{\omega}(\mu)$ to the subspace $\mathcal{P}^{\infty}(X)$ of volume forms in $\mathcal{M}_{1}(X)$ may be represented by $\varphi_{\mu}$ :

$$
d E_{\omega}(\mu)=-\varphi_{\mu}
$$

(in this special case the formula follows by standard Legendre duality, since formula 3.14 expresses $E(\mu)$ as the Legendre transform of the functional-E $(-u))$. One can then recover $E_{\omega}$ on all of $\mathcal{M}_{1}(X)$ as the greatest lower semi-continuous from $\mathcal{P}^{\infty}(X)$. When $\omega \geq 0$ the following explicit formula for $E_{\omega}$ on $\mathcal{P}^{\infty}(X)$ follows from formula 3.9 (and integration by parts):

$$
E_{\omega}(\mu)=\frac{1}{V} \sum_{j=0}^{n-1} \frac{1}{j+2} \int d \varphi_{\mu} \wedge d^{c} \varphi_{\mu} \wedge \frac{\omega_{\varphi_{\mu}}^{j}}{j !} \wedge \frac{\omega^{n-1-j}}{(n-1-j) !},
$$

Moreover, $E_{\omega}(\mu)$ coincides with $\left(I_{\omega}-J_{\omega}\right)\left(\varphi_{\mu}\right)$, in terms of Aubin's functionals $I$ and $J[6]$. 
3.7. Compactification of $\mathbb{C}^{n}$ by $\mathbb{P}^{n}$. We recall that the $n$-dimensional complex projective space

$$
\left.\mathbb{P}^{n}:=\mathbb{C}^{n+1}-\{0\} / \mathbb{C}^{*}\right)
$$

comes with a tautological line bundle whose total space, with the zero-section deleted, is $\mathbb{C}^{n+1}-\{0\}$ : the line over a point $\left[Z_{0}: \ldots .: Z_{m}\right] \in \mathbb{P}^{n}$ is simply the line $\mathbb{C}\left(Z_{0}, . ., Z_{n}\right)$. The dual of the tautological line bundle is called the hyperplane line bundle and is usually denoted by $\mathcal{O}(1)$. The notation reflects the fact that the space $H^{0}\left(\mathbb{P}^{n}, \mathcal{O}(1)\right)$ of holomorphic sections of $\mathcal{O}(1) \rightarrow \mathbb{P}^{n}$ may be identified with the space of 1 -homogeneous holomorphic functions $F$ on $\mathbb{C}^{n+1}$ (by the correspondence in Section 3.2.1). In particular, the homogeneous coordinates $Z_{i}$ on $\mathbb{P}^{n}$ define holomorphic sections of $\mathcal{O}(1) \rightarrow \mathbb{P}^{n}$. Similarly, the metrics $\|\cdot\|$ on $\mathcal{O}(1)$ may be identified with positively 1 -homogeneous non-negative functions $N$ on $\mathbb{C}^{n+1}$ (assumed strictly positive on $\left.\mathbb{C}^{n+1}-\{0\}\right)$. Accordingly,

$$
\|F\|=\frac{|F|}{N}
$$

descends from $\mathbb{C}^{n+1}$ to a well-defined function on $\mathbb{P}^{n+1}$. In particular, the norm $N$ defined by the Euclidean norm on $\mathbb{C}^{n+1}$ induces a metric on $\mathcal{O}(1)$, called the Fubini-Study metric, that we shall denote by $\|\cdot\|_{F S}$. Its curvature form $\omega_{F S}$ defines a Kähler form on $\mathbb{P}^{n}$ (as follows from the fact that the squared Euclidean norm $N^{2}$ defines a smooth and strictly plurisubharmonic function on $\left.\mathbb{C}^{n+1}\right)$. Thus $\left(\mathbb{P}^{n}, \mathcal{O}(1)\right)$ is a polarized manifold.

Following standard practice we will identify $\mathbb{C}^{n}$ with the open subset

$$
U_{0}:=\mathbb{P}^{n}-H_{0}, \quad H_{0}:=\left\{Z_{0}=0\right\},
$$

i.e. with the image of the embedding

$$
\mathbb{C}^{n} \rightarrow \mathbb{P}^{n}, \quad z \mapsto[1: z]
$$

Over $U_{0}$ the line bundle $\mathcal{O}(1)$ is trivialized by $Z_{0}$ (since it is non-vanishing precisely on $U_{0}$ ). Accordingly, over $U_{0}$ the Fubini-Study metric $\|\cdot\|_{F S}$ is represented by the weight

$$
\phi_{F S}(z):=-\log \left\|Z_{0}\right\|_{F S}^{2}:=-\log \frac{\left|Z_{0}\right|^{2}}{\left|Z_{0}\right|^{2}+\ldots+\left|Z_{n}\right|^{2}}=\log \left(1+|z|^{2}\right)
$$

defining a smooth metric with strictly positive curvature form $\omega_{F S}$.

The space $H^{0}\left(\mathbb{P}^{n}, k \mathcal{O}(1)\right)$ may be naturally identified with the space of all homogeneous polynomials $P_{k}$ of degree $k$ in $\mathbb{C}^{n+1}$ (by the correspondence in Section 3.2.1) and

$$
\left\|P_{k}\right\|^{2}([Z])=\frac{\left|P_{k}(Z)\right|}{N(Z)^{k}}
$$

Hence, "homogenization" establishes a one-to-one correspondence

$$
\mathcal{P}_{k}\left(\mathbb{C}^{n}\right) \longleftrightarrow\left(H^{0}\left(\mathbb{P}^{n}, k \mathcal{O}(1)\right), p_{k}\left(z_{1}, \ldots z_{n}\right)=P_{k}\left(1, z_{1}, \ldots, z_{n}\right)\right.
$$

between the space $\mathcal{P}_{k}\left(\mathbb{C}^{n}\right)$ of all polynomials $p_{k}$ on $\mathbb{C}^{k}$ of degree at most $k$ and $H^{0}\left(\mathbb{P}^{n}, k \mathcal{O}(1)\right)$. In particular, over $U_{0}\left(=\mathbb{C}^{n}\right)$ we can write

$$
P_{k}=p_{k} Z_{0}^{\otimes k}
$$

Lemma 3.11. Setting

$$
\phi(z):=-\log \left\|Z_{0}\right\|^{2}
$$

yields a correspondence between 
- metrics $\|\cdot\|$ on $\mathcal{O}(1) \rightarrow \mathbb{P}^{n}$ with positive curvature current and functions $\phi(z)$ in the Lelong class $\mathcal{L}\left(\mathbb{C}^{n}\right)$ (formula 2.12)

- usc metrics $\|\cdot\|$ on $\mathcal{O}(1) \rightarrow \mathbb{P}^{n}$ (i.e. the local weights are lsc) and lsc functions $\phi(z)$ on $\mathbb{C}^{n}$ with super logarithmic growth, i.e. $\phi(z) \geq \log \left(1+|z|^{2}\right)+O(1)$.

Proof. Let us start with the second point. Consider a lsc function $\phi(z)$ on $\mathbb{C}^{n}$ satisfying the growth property in the lemma. This means that $u(z):=\phi(z)-\phi_{F S}(z)$ is a lsc function on $U:=\mathbb{P}^{n}-H_{0}$ which is uniformly bounded from below. Now, any lsc function $u$ on a subset $U$ in a topological space admits, if $u$ is bounded from below on $U$, a canonical lsc extension $\bar{u}$ to the closure $\bar{U}$, namely the greatest lsc extension of $u$. The corresponding metric on $\mathcal{O}(1) \rightarrow \mathbb{P}^{n}$ is then defined as $\|\cdot\|_{u}:=\|\cdot\|_{F S} e^{-\bar{u} / 2}$, which proves the first point. The proof of the first point is similar, using that a function $u$ is $\omega$-psh and bounded from above on $X-A$, where $A$ is a pluripolar subset, iff the smallest usc of $u$ to $X$ is $\omega$-psh. This follows directly from the corresponding classical local property of psh functions.

The correspondence above is made so that

$$
\left|p_{k}(z)\right|^{2} e^{-k \phi}=\left\|P_{k}(1, z)\right\|^{2},
$$

as follows directly from formula 3.17 .

Remark 3.12. If $X$ is a complex submanifold of $\mathbb{P}^{m}$ (which, by Chow's theorem, equivalently means that $X$ is a projective non-singular algebraic variety), then $\left(X, \mathcal{O}_{X}(1)\right)$ is a polarized manifold, where $\mathcal{O}(1)_{X}$ denotes the restriction of $\mathcal{O}(1) \rightarrow \mathbb{P}^{m}$ to $X$. Indeed, the restriction to $\mathcal{O}_{X}(1)$ of the Fubini-Study metric on $\mathcal{O}(1)$ has strictly positive curvature. Conversely, by the Kodaira embedding theorem, if $(X, L)$ is a polarized manifold, then after perhaps replacing $L$ by a large tensor power, $X$ may be holomorphically embedded in a projective space $\mathbb{P}^{m}$ in such a way that $L$ gets identified with $\mathcal{O}(1)_{X}$ and $H^{0}(X, k L)$ identifies with the restriction to $X$ of the space of all $k$-homogeneous polynomials over $\mathbb{P}^{m}$. This means that a line bundle is positive iff it is ample, in the algebro-geometric sense.

3.7.1. The (non-)weighted pluricomplex energy $E$ in $\mathbb{C}^{n}$. Let $\mu$ be a probability measure on $\mathbb{C}^{n}$ such that

$$
\int_{\mathbb{C}^{n}} \log \left(1+|z|^{2}\right) \mu<\infty
$$

We define the non-weighted pluricomplex energy $E(\mu)$ by

$$
E(\mu)=\sup _{\psi \in \mathcal{E}^{1}\left(\mathbb{C}^{n}\right)}\left(\mathcal{E}(\psi)-\mathcal{E}\left(\psi_{T^{n}}\right)-\int_{\mathbb{C}^{n}} \psi \mu\right),
$$

where we have identified $\mathcal{E}$ with a functional on the Lelong class $\mathcal{L}\left(\mathbb{C}^{n}\right)$, using the correspondence in Lemma 3.11. We note that the extremal function $\psi_{T^{n}}$ is explicitly given by

$$
\psi_{T^{n}}=\log \max _{i=1, \ldots, n}\left\{1,\left|z_{i}\right|^{2}\right\} .
$$

Alternatively, we could directly have defined $\mathcal{E}$ (up to an additive constant) on $\mathcal{L}\left(\mathbb{C}^{n}\right)$ by requiring that

$$
d \mathcal{E}_{\mid \psi}=\left(d d^{c} \psi\right)^{n}
$$

when $\psi$ is psh, locally bounded and with logarithmic growth (note that the definition 3.19 is independent of the choice of additive constant for $\mathcal{E}$ ). 
Assume now that $\mu \in \mathcal{M}_{1}\left(\mathbb{C}^{n}\right)$ has compact support. Embedd $\mathbb{C}^{n}$ in $\mathbb{P}^{n}$ as above and fix an auxiliary smooth metric $\|\cdot\|$ on $\mathcal{O}(1) \rightarrow \mathbb{P}^{n}$ coinciding with the trivial metric on a bounded neighborhood $U$ of the support of $\mu$. In particular, the curvature form $\omega$ vanishes on $U$.

Lemma 3.13. Assume that $\mu \in \mathcal{M}_{1}\left(\mathbb{C}^{n}\right)$ has compact support. The non-weighted pluricomplex energy $E(\mu)$ may be expressed as

$$
E(\mu):=E_{\omega}(\mu)-\mathcal{E}_{\omega}\left(P_{T^{n}} 0\right)
$$

In particular, if $K$ is a subset of the unit-polydisc, then $\omega$ can be taken as $\omega_{\psi_{T^{n}}}$ and then

$$
E(\mu):=E_{\omega_{T^{n}}}(\mu)
$$

Moreover, $E(\mu)$ coincides with the classical energy 2.3 when $n=1$.

Proof. The first formula follows directly from making the identifications in Section 3.7 and by rewriting

$$
\int_{\mathbb{C}^{n}} \psi \mu=\int_{\mathbb{C}^{n}}\left(\psi-\psi_{T^{n}}\right) \mu+\int_{\mathbb{C}^{n}} \psi_{T^{n}} \mu
$$

where the last term is finite, by assumption. Moreover, when $n=1$ we can express the classical logarithmic energy as

$$
-\frac{1}{2} \int_{\mathbb{C}} \log |z-w|^{2} \mu(w) \otimes \mu(w)=-\frac{1}{2} \int_{\mathbb{C}} \psi_{\mu} \mu, \quad \psi_{\mu}(z)=\int_{\mathbb{C}}\left(\log |z-w|^{2}-\phi(z)-\phi(w)\right) \mu
$$

where $d d^{c} \psi_{\mu}+\omega=\mu$, using that $\omega=d d^{c} \phi$ with $\phi=0$ close to the support of $\mu$. Hence, the logarithmic energy coincides with $E_{\omega}(\mu)$ up to an overall additive constant. This means that it also coincides with the non-weighted pluricomplex energy $E(\mu)$ up to an overall constant $C$. Finally, the constant $C$ can be seen to be equal to 0 by taking $\mu=d \theta$, the uniform measure on $S^{1}$.

Given a continuous function $\phi$ on $\mathbb{C}^{n}$ with super logarithmic growth (formula 3.6), we consider the corresponding weighted pluricomplex energy $E_{\phi}$ on $\mathcal{M}_{1}\left(\mathbb{C}^{n}\right)$,

$$
E_{\phi}(\mu)=E_{\omega_{T^{n}}}(\mu)+\int\left(\phi-\psi_{T^{n}}\right) \mu
$$

This is well-defined since both terms above take values in $]-\infty, \infty]$. In particular, if $\mu$ satisfies the intractability property 3.18 , then we can decompose

$$
E_{\phi}(\mu):=E(\mu)+\int \phi \mu .
$$

Remark 3.14. It is enough to assume that $\phi$ is lsc (and has super-logarithmic growth) in order to define $E_{\phi}(\mu)$, as above. But in order to ensure that $E_{\phi}$ is not identically equal to infinity one then has to assume that $\{\phi<\infty\}$ is an open non-pluripolar subset.

\section{Probabilistic SETUP AND MEAN FIELd APPROXimations}

Given a Hausdorff locally compact topological space $\Omega$ we will denote by $\mathcal{M}(\Omega)$ the space of all signed (Borel) measures on $\Omega$ and by $\mathcal{M}_{1}(\Omega)$ the subspace of all measures with unit total mass, i.e. the space of probability measures on $\Omega$. 
4.1. Gamma-convergence and Legendre-Fenchel transforms. We start with some functional analytic preparations in the context of Gamma-convergence, as introduced by De Georgi (see the book 38] for background on Gamma-convergence).

Definition 4.1. A sequence of functions $E_{N}$ on a topological space $\mathcal{M}$ is said to $\Gamma$-converge to a function $E$ on $\mathcal{M}$ if

$$
\begin{array}{ccc}
\mu_{N} \rightarrow \mu \text { in } \mathcal{M} & \Longrightarrow & \liminf _{N \rightarrow \infty} E_{N}\left(\mu_{N}\right) \geq E(\mu) \\
\forall \mu & \exists \mu_{N} \rightarrow \mu \text { in } \mathcal{M}: & \lim _{N \rightarrow \infty} E_{N}\left(\mu_{N}\right)=E(\mu)
\end{array}
$$

Given $\mu$ a sequence $\mu_{N}$ as in the last point above is called a recovery sequence. The limiting functional $E$ is automatically lower semi-continuous on $\mathcal{M}$.

In the present setting we will take $\mathcal{M}=\mathcal{M}(X)$ for a compact manifold $X$ and define $E_{N}$ by setting $E_{N}=\infty$ on the complement of the image of the map $\delta_{N}$ and

$$
E_{N}\left(\delta_{N}\left(x_{1}, \ldots, x_{N}\right)\right):=H^{(N)}\left(x_{1}, \ldots, x_{N}\right) / N
$$

Remark 4.2. Note that in the previous example it is not the case that $\mu_{N}:=\delta_{N}\left(x_{1}, \ldots, x_{N}\right) \rightarrow \mu$ implies that $\lim \sup _{N} E_{N}\left(\mu_{N}\right) \leq E(\mu)$. Indeed, for any sequence where two points, say $x_{1}$ and $x_{2}$, coincide, $E_{N}\left(\mu_{N}\right)=\infty$ ! This phenomenon explains the asymmetry between the first and the second condition in the definition of Gamma-convergence.

A criterion for Gamma-convergence on $\mathcal{M}_{1}(X)$ can be obtained using duality in topological vector spaces, as next explained. Let $f$ be a function on a topological vector space $V$. The Legendre-Fenchel transform $f^{*}$ of $f$ is defined as following convex lower semi-continuous function $f^{*}$ on the topological dual $V^{*}$

$$
f^{*}(w):=\sup _{v \in V}\langle v, w\rangle-f(v)
$$

in terms of the canonical pairing between $V$ and $V^{*}$. In the present setting we will take $V=C^{0}(X)$ and $V^{*}=\mathcal{M}(X)$, the space of all signed Borel measures on a compact topological space $X$.

Proposition 4.3. Let $E_{N}$ be a sequence of functions on the space $\mathcal{M}_{1}(X)$ of probability measures on a compact space $X$ and assume that

$$
\lim _{N \rightarrow \infty} E_{N}^{*}(w)=f(w)
$$

for any $w \in C(X)$ and that $f$ defines a Gateaux differentiable function on $C(X)$. Then $E_{N}$ converges to $E:=f^{*}$ in the sense of $\Gamma$-convergence on the space $\mathcal{M}_{1}(X)$, equipped with the weak topology.

See [18] for the proof (the existence of a recovery sequence is based on an application of a general approximation result of Brøndsted-Rockafellar, which applies in certain topological vector spaces).

Remark 4.4. In the present setting it will convenient to rather look at the following transform

$$
-E_{N}^{*}(-u)=\inf _{X^{N}} N^{-1}\left(H^{(N)}\left(x_{1}, \ldots, x_{N}\right)+\sum_{i=1}^{N} u\left(x_{i}\right)\right)
$$


4.2. Probabilistic setup. We start by recalling some basic notions of probability theory (covered by any standard textbook; see in particular [53] for an introduction to large deviation techniques). A probability space is a space $\Omega$ equipped with a probability measure $p$. The space $\Omega$ is called the sample space and a measurable subset $\mathcal{B} \subset \Omega$ is called an event with

$$
\operatorname{Prob} \mathcal{B}:=p(\mathcal{B})
$$

interpreted as the probability of observing the event $\mathcal{B}$ when sampling from $(\mathcal{X}, \Omega)$. A measurable function $Y: \Omega \rightarrow \mathcal{Y}$ on a probability space $(\Omega, p)$ is called a random element with values in $\mathcal{Y}$ and its law $\Gamma$ is the probability measure on $\mathcal{Y}$ defined by the push-forward measure

$$
\Gamma:=Y_{*} p
$$

(the law of $Y$ is often also called the distribution of $Y$ ). A sequence of random elements $Y_{N}: \Omega_{N} \rightarrow \mathcal{Y}$ taking values in the same topological space $\mathcal{Y}$ are said to convergence in law towards a deterministic element $y$ in $\mathcal{Y}$ if the corresponding laws $\Gamma_{N}$ on $\mathcal{Y}$ converge to a Dirac mass at $y$ :

$$
\lim _{N \rightarrow \infty} \Gamma_{N}=\delta_{y}
$$

in the weak topology. In the present setting $\mathcal{Y}$ will always be a separable metric space with metric $d$ and then $Y_{N}$ converge in law towards the deterministic element $y$ iff $Y_{N}$ converge in probability towards $y$, i.e. for any fixed $\epsilon>0$

$$
\lim _{N \rightarrow \infty} p\left\{d\left(Y_{N}, y\right)>\epsilon\right\}=0 .
$$

Remark 4.5. The expectation of a random variable $Y$ it defined by

$$
\mathbb{E}(Y):=\int_{\Omega} Y p
$$

(aka the sample mean of $Y$ ) which defines an element in $\mathcal{Y}$. The statement that $Y_{N}$ converges in law towards a deterministic element $y$ equivalently means that $\mathbb{E}\left(Y_{N}\right) \rightarrow y$ and that $Y_{N}$ satisfies the (weak) law of large numbers, i.e. the probability that $Y_{N}$ deviates from its mean tends to zero, as $N \rightarrow \infty$.

A random point process with $N$ particles on a space $X$ is, by definition, a probability measure $\mu^{(N)}$ on the $N$-fold product $X^{N}$ (the $N$-particle space) which is symmetric, i.e. invariant under action of the group $S_{N}$ by permutations of the factors of $X^{N}$. The empirical measure of a given random point process is the following random measure

$$
\delta_{N}: X^{N} \rightarrow \mathcal{M}_{1}(X),\left(x_{1}, \ldots, x_{N}\right) \mapsto \delta_{N}\left(x_{1}, \ldots, x_{N}\right):=\frac{1}{N} \sum_{i=1}^{N} \delta_{x_{i}}
$$

on $\left(X^{N}, \mu^{(N)}\right)$. The law of $\delta_{N}$ thus defines a probability measure on the space $\mathcal{M}_{1}(X)$, that we shall denote by $\Gamma_{N}$ :

$$
\Gamma_{N}:=\left(\delta_{N}\right)_{*} \mu^{(N)}
$$

Remark 4.6. The $j$-point correlation measure $\left(\mu^{(N)}\right)_{j}$ of the $N$-particle random point process is the probability measure on $X^{j}$ defined as the push-forward to $X^{j}$ of $\mu^{(N)}$ under projection $X^{N} \rightarrow X^{j}$, where $\left(x_{1}, \ldots, x_{N}\right) \mapsto\left(x_{i_{1}}, \ldots, x_{i_{j}}\right)$ for any choice of $j$ different indices $i_{1}, \ldots, i_{j}$. In particular,

$$
\mathbb{E}\left(\delta_{N}\right)=\left(\mu^{(N)}\right)_{1}
$$


In the statistical mechanical setup recalled in the beginning of Section 2.3 a random point process on with $N$ points on $X$ is induced by the data $\left(H^{(N)}, d V, \beta\right)$. We denote by

$$
\mu_{\beta}^{(N)}=\frac{1}{Z_{N, \beta}} e^{-\beta H^{(N)}} d V^{\otimes N}
$$

the corresponding Gibbs measure, which defines a symmetric probability measure on $X^{N}$.

4.2.1. Large Deviation Principles (LDP). The notion of a Large Deviation Principle (LDP), introduced by Varadhan, allows one to give a notion of exponential convergence in probability. The general definition of a Large Deviation Principle (LDP) for a general sequence of measures [53] is modeled on the classical Laplace principle of "saddle approximation":

Definition 4.7. Let $\mathcal{Y}$ be a Polish space, i.e. a complete separable metric space.

(i) A function $I: \mathcal{Y} \rightarrow]-\infty, \infty]$ is a rate function if it is lower semi-continuous. It is a good rate function if it is also proper.

(ii) A sequence $\Gamma_{k}$ of measures on $\mathcal{Y}$ satisfies a large deviation principle with speed $r_{k}$ and rate function $I$ if

$$
\limsup _{k \rightarrow \infty} \frac{1}{r_{k}} \log \Gamma_{k}(\mathcal{F}) \leq-\inf _{\mu \in \mathcal{F}} I
$$

for any closed subset $\mathcal{F}$ of $\mathcal{Y}$ and

$$
\liminf _{k \rightarrow \infty} \frac{1}{r_{k}} \log \Gamma_{k}(\mathcal{G}) \geq-\inf _{\mu \in G} I(\mu)
$$

for any open subset $\mathcal{G}$ of $\mathcal{Y}$.

In the present setting $\Gamma_{N}$ will arise as the sequence of probability measures on $\mathcal{M}_{1}(X)$ defined as laws of the empirical measures $\delta_{N}$ (formula 4.3). Once the LDP has been established we can apply the following basic

Lemma 4.8. Assume that the laws $\Gamma_{N}$ above satisfy a LDP with a good rate functional $I_{\beta}$ which admits a unique minimizer $\mu_{\beta}$. Then the random measures $\delta_{N}$ converge in law towards the deterministic measure $\mu_{\beta}$. More precisely,

$$
\operatorname{Prob}\left\{d\left(\delta_{N}, \mu_{\beta}\right) \geq \epsilon\right\} \leq C_{\epsilon} e^{-N / C_{\epsilon}}
$$

Proof. First applying the LDP to $\mathcal{F}=\mathcal{G}=\mathcal{Y}$ gives $I_{\beta}\left(\mu_{\beta}\right)=0$. Since $\mu_{\beta}$ is the unique minimizer of $I_{\beta}$ it follows that $\inf I_{\beta}>0$ on the closed subset $\mathcal{F}_{\epsilon}$ of $\mathcal{Y}$ where $d\left(\cdot, \mu_{\beta}\right) \geq \epsilon$. Applying $(i)$ in the LDP to $\mathcal{F}_{\epsilon}$ thus concludes the proof of the desired. deviation inequality.

In other words, the lemma says that risk that $\delta_{N}$ deviates from $\mu_{\beta}$ is exponentially small. In order to establish the LDP we will have great use for the following alternative formulation of a LDP (see Theorems 4.1.11 and 4.1.18 in [53]):

Proposition 4.9. Let $\mathcal{Y}$ be a compact metric space and denote by $B_{\epsilon}(y)$ the ball of radius $\epsilon$ centered at $y \in \mathcal{Y}$. Then a sequence $\Gamma_{N}$ of probability measures on $\mathcal{P}$ satisfies a LDP with speed $r_{N}$ and a rate functional I iff

$$
\lim _{\epsilon \rightarrow 0} \liminf _{N \rightarrow \infty} \frac{1}{r_{N}} \log \Gamma_{N}\left(B_{\epsilon}(y)\right)=-I(y)=\lim _{\epsilon \rightarrow 0} \limsup _{N \rightarrow \infty} \frac{1}{r_{N}} \log \Gamma_{N}\left(B_{\epsilon}(y)\right)
$$


In the present setting of a sequence of random point process with $N$ particles the previous proposition may be symbolically summarized as follows:

$$
\operatorname{Prob}\left(\frac{1}{N} \sum_{i=1}^{N} \delta_{x_{i}} \in B_{\epsilon}(\mu)\right) \sim e^{-r_{N} I(\mu)}
$$

when first $N \rightarrow \infty$ and then $\epsilon \rightarrow 0$.

We will also use the following classical result of Sanov, which is the standard example of an LDP for random point processes (describing the case when the particles $x_{1}, \ldots, x_{N}$ define independent variables with identical distribution $\left.\mu_{0}\right)$ :

Proposition 4.10. (Sanov) Let $X$ be a topological space and $\mu_{0}$ a finite measure on $X$. Then the laws $\Gamma_{N}$ of the empirical measures $\delta_{N}$ defined with respect to the product measure $\mu_{0}^{\otimes N}$ on $X^{N}$ satisfy an LDP with speed $N$ and rate functional the relative entropy $D_{\mu_{0}}$.

We recall that the relative entropy $D_{\mu_{0}}$ (also called the Kullback-Leibler divergence or the information divergence in probability and information theory) is the functional on $\mathcal{M}_{1}(X)$ defined by

$$
D_{\mu_{0}}(\mu):=\int_{X} \log \frac{\mu}{\mu_{0}} \mu
$$

when $\mu$ has a density $\frac{\mu}{\mu_{0}}$ with respect to $\mu_{0}$ and otherwise $D_{\mu_{0}}(\mu):=\infty$. If $\mu_{0}$ is a probability measure, then $D_{\mu_{0}}(\mu) \geq 0$ and $D_{\mu_{0}}(\mu)=0$ iff $\mu=\mu_{0}$ (by Jensen's inequality).

Remark 4.11. The "physical entropy" is usually defined as

$$
S(\mu):=-D_{\mu_{0}}(\mu)
$$

In fact, Sanov's theorem can be seen as a mathematical justification of Boltzmann's original formula expressing the entropy $S$ as the logarithm of the number of microscopic states consistent with a given macroscopic state (using the characterization of a LDP in Prop 4.9).

4.3. Determinantal point processes, their deformations and random matrices. In this section we compare the definition of the probability measure 2.22 with the general setup of determinantal point processes [73]. A brief comparison with random matrix theory is also made.

Let $(M, \nu)$ be a measure space. A random point process with $N$ particles on $M$ is said to be a projectional determinantal point processes if there exists a complex-valued Hermitian function $\mathcal{K}(x, y)$, which defines a projection operator on $L^{2}(X, \nu)$ of $\operatorname{rank} N$ on $M \times M$, such that

$$
\mu^{(N)}=\frac{1}{N !} \operatorname{det}\left(\mathcal{K}\left(x_{i}, x_{j}\right)\right)_{i, j \leq N} \nu^{\otimes N}
$$

(the kernel $\mathcal{K}$ need only be defined a.e. wrt $\nu \otimes \nu$ ). In the most general setup of determinantal point processes it is not assumed that $\mathcal{K}$ defines a projection operator, but this will always be assumed in the following and we will thus drop the adjective projectional. A remarkable feature of a determinantal point process is that all correlation measures 4.6 can be expressed as determinants of the kernel $\mathcal{K}$ and, in particular,

$$
\left(\mu^{(N)}\right)_{1}=\mathbb{E}\left(\delta_{N}\right)=\frac{1}{N} \mathcal{K}(x, x) \nu
$$

Moreover, 


$$
\operatorname{det}\left(\mathcal{K}\left(x_{i}, x_{j}\right)\right)_{i, j \leq N}=\left.|| f\left(x_{1}, \ldots, x_{N}\right)\right|^{2},
$$

where

$$
f\left(x_{1}, \ldots, x_{N}\right)=\operatorname{det}\left(f_{i}\left(x_{j}\right)\right)_{i, j \leq N_{k}}
$$

expressed in terms of a fixed orthonormal base $f_{1}, \ldots, f_{N}$ in the Hilbert space $\mathcal{H}_{N} \subset L^{2}(X, \nu)$ defined as the image of the projection operator. More generally, if $f_{i}$ is a, possibly nonorthonormal, basis in $\mathcal{H}_{N}$, then

$$
\mu^{(N)}=\left.\frac{1}{Z_{N}}|| f\left(x_{1}, \ldots, x_{N}\right)\right|^{2} \nu^{\otimes N}, Z_{N}:=\left.\int_{X^{N}}|| f\left(x_{1}, \ldots, x_{N}\right)\right|^{2} \nu^{\otimes N}
$$

The systematic study of determinantal point processes was initiated by Macchi in the seventies who called them fermionic point processes, inspired by the properties of fermion gases in quantum mechanics. From the physics point of view the functions $f_{i}$ play the role of wave functions representing single fermions and $f$ (called a Slater determinant) is the corresponding $N$-body wave function (which is totally anti-symmetric, since the particles are fermions).

Example 4.12. The Vandermonde probability measure 2.22 defines, when $\beta=k$, a (projectional) determinantal random point process on $\mathbb{C}^{n}$ with $N_{k}$ particles, $\nu=d V$ and $\mathcal{H}_{N_{k}}$ the space of all weighted polynomials $p_{k} e^{-k \phi / 2}$ of degree at most $k$. In physical terms $p_{k} e^{-k \phi / 2}$ represents a fermion (electron) on $\mathbb{C}^{n}$ subject to an exterior magnetic field represented by the two-form $k \omega^{\phi}$. Indeed, $p_{k} e^{-k \phi / 2}$ is in the null space of the Dirac operator coupled to a gauge field $A$ with curvature form $d A=i \omega^{\phi}$ (see [13] for the relation to boson-fermion correspondences). When $n=1$ the corresponding probability measures appear in the collective description of the integer Quantum Hall Effect (QHE) introduced by Laughlin, while the case when $\beta=\mathrm{km}$ for an integer $m$ appears in the fractional QHE (see the survey [78]).

Random Matrix Theory offers several famous examples of determinantal point processes (see the book [91] for further background):

Example 4.13. Consider a random Hermitian matrix of rank $N$, where the entries are taken to be independent and normally distributed. Then the corresponding eigenvalue distribution defines a determinantal point process on $\mathbb{R}$. More precisely, if $\gamma_{N}$ denotes the centered Gaussian measure of variance $N^{-1}$ on the space $H_{N}$ of all Hermitian matrices of rank $N$, then the push-forward of $\gamma_{N}$ to $\mathbb{R}^{N}$ (obtained by fixing an ordering of the eigenvalues) is given by the determinantal point process on $X:=\mathbb{R}$ defined by the Vandermonde expression 2.22 for $n=1$ and $\beta=k$ and the weight $\phi(x):=|x|^{2} / 2$, but with the measure $d V$ on $\mathbb{C}$ replaced by the Lesbegue measure on $\mathbb{R} \subset \mathbb{C}$. The corresponding limiting equilibrium measure $\mu_{(\phi, \mathbb{R})}$ is the semi-circle law, $\mu_{(\phi, \mathbb{R})}=1_{[-1,1]}\left(1-|x|^{2}\right)^{1 / 2} 2 / \pi$. The case when $\phi(z):=|z|^{2} / 2$ and $d V$ is Lebesgue measure on $\mathbb{C}$ also appears naturally in random matrix theory as the eigenvalue distribution of a general rank $N$ matrix with random complex entries, i.e. the centered Gaussian measure of variance $N^{-1}$ on $g l(N, \mathbb{C}$ ) (this is called the Ginibre ensemble). In this case $\mu_{\phi}$ is the uniform probability measure on the unit-disc. Moreover, if $(A, B) \in \operatorname{gl}(N, \mathbb{C})^{2}$ are drawn independently from the Ginibre ensemble, then the eigenvalues of $A B^{-1}$ are distributed according to the probability measure 2.22 with $\phi=\phi_{F S}$ and $d V=d \lambda$ on $\mathbb{C}\left[80\right.$ ] (and then $\mu_{\phi}$ may be identified with the invariant probability measure on the Riemann sphere). 
Given a positive number $p$ one can consider a more general class of point processes by setting

$$
\mu_{p}^{(N)}=\frac{1}{Z_{p}} \operatorname{det}\left(\mathcal{K}\left(x_{i}, x_{j}\right)\right)_{i, j \leq N}^{p / 2} \nu^{\otimes N}
$$

which specializes to the determinantal case when $p=2$. Equivalently, this means that $\mu^{(N)}$ can be expressed as

$$
\mu_{p}^{(N)}=\left.\frac{1}{Z_{N, p}}|| f\left(x_{1}, \ldots, x_{N}\right)\right|^{p} \nu^{\otimes N}, Z_{N, p}:=\left.\int_{X^{N}}|| f\left(x_{1}, \ldots, x_{N}\right)\right|^{p} \nu^{\otimes N},
$$

which suggests that this setting can be viewed as an $L^{p}$-generalization of determinantal point process. In the random matrix literature one usually writes $p=\beta$ and calls $\left(M^{N}, \mu_{p}^{(N)}\right)$ a $\beta$-ensemble or a $\beta$-deformation of a determinantal point process (the parameter $\beta$ is called the Dyson index). In the present setting in $\mathbb{C}^{n}$, introduced in Section 2.3, it is, however, convenient to use a rescaling and set

$$
p:=2 \beta / k,
$$

Thus the determinantal case appears when $\beta$ is taken to be depend on $k$ as $\beta=k$.

Example 4.14. The cases $p=1,2$ and 4 with $\phi(x)=|x|^{2} / 2$ on the real line appear naturally in Random Matrix Theory, as Gaussian ensembles GOE, GUE and GSE [91]. They are classified under their symmetry group (the Orthogonal, Unitary and Symplectic group, respectively). More generally, for any $p>0$ random matrix realizations were introduced in [55], in the case of real eigenvalues, based on random Jacobi matrices. Using such realization the large $N$-limit with $p=\beta N^{-1}$ was studied in [57], proving the analog of Theorem 2.7 for $n=1$ (and identifying the limiting measure $\mu_{\beta}$ with the spectral measure of a semi-infinite Jacobi matrix). However, random matrix interpretations in the complex case do not seem to be known, for $p \neq 2$.

4.3.1. Determinantal point process for polarized manifolds. Determinantal point processes may also be attached to a polarized complex manifold $(X, L)$ when $L$ is endowed with an Hermitian metric $\|\cdot\|$ and a $X$ with a measure $\nu$, which is assumed to not charge pluripolar subsets. The corresponding large $N$-limit was studied in [13]. To explain the setup we endow the $N_{k}$-dimension Hilbert space $H^{0}(X, k L)$ with the $L^{2}$-norm induced from $(\nu,\|\cdot\|)$ and denote by $K_{k}$ the corresponding Bergman kernel. In other words, $K_{k}$ is the holomorphic section of $k L \otimes k \bar{L} \rightarrow X \times \bar{X}$ defined as the integral kernel of the orthogonal projection from $L^{2}(X, L)$ onto $H^{0}(X, k L)$. The corresponding determinantal probability measure on $\mu^{\left(N_{k}\right)}$ on $X^{N_{k}}$ may be defined as

$$
\mu^{(N)}=\frac{1}{N !}\left\|\operatorname{det}\left(K_{k}\left(x_{i}, x_{j}\right)\right)_{i, j \leq N}\right\| \nu^{\otimes N}
$$

To see that this fits into the definition 4.8, fix $e_{k} \in H^{0}(X, k L)$, not vanishing identically on $X$, and denote by $U$ the subset of $X$ where $e_{k}$ is non-vanishing. On $U$ we can write $K_{k}=K_{k}(x, y) e_{k} \otimes \bar{e}_{k}$, where $K_{k}(x, y)$ defines a holomorphic function on $X \times \bar{X}$ and set

$$
\mathcal{K}(x, y):=K_{k}(x, y)\left\|e_{k}\right\|(x)\left\|e_{k}\right\|(y),
$$

which is defined a.e. wrt $\nu \otimes \nu$ (since, by assumption, $\nu$ does not charge pluripolar subsets), as desired. As a consequence, by formula 4.10,

$$
\mathbb{E}\left(\delta_{N}\right)=\rho_{k} \nu, \rho_{k}:=\frac{1}{N}\left\|K_{k}(x, x)\right\|
$$


where the right hand side is called the Bergman measure in [27] and $\rho_{k}$ is called the Bergman density function. It admits the alternative representation

$$
\rho_{k}(x)=N_{k}^{-1} \sup _{\Psi \in H^{0}(X, k L)} \frac{\|\Psi(x)\|^{2}}{\int_{X}\|\Psi\|^{2} \nu}
$$

Example 4.15. When $(X, L)=\left(\mathbb{P}^{n}, \mathcal{O}(1)\right)$ and $e_{k}$ is the $k$ th tensor power of the section $Z_{0}$ the corresponding determinantal point process on $M:=\mathbb{C}^{n}$ is precisely the one defined described in example 4.12

The corresponding deformed determinatal point processes will be studied in Section 5 and referred to as temperature-deformed determinantal point processes, since $\beta$ plays the role of the inverse temperature.

4.4. Mean field approximations and large deviations. The notion of mean field approximations (and mean field theory) is wide-spread in the physics literature and first appeared in the study of phase transitions in ferromagnetic spins system (see the Appendix). But here the notion will be used in a generalized sense, which goes beyond the more standard "linear" setting of pair-interactions encountered in the physics literature.

Let us start with some heuristic arguments. Assume that the following "Mean Field Approximation" holds:

$$
E^{(N)}\left(x_{1}, \ldots x_{N}\right):=\frac{1}{N} H^{(N)}\left(x_{1}, \ldots, x_{N}\right) \approx E\left(\frac{1}{N} \sum_{i=1}^{N} \delta_{x_{i}}\right), \quad N>>1
$$

for some functional $E$ on $\mathcal{M}_{1}(X)$.

By definition, given $\mu \in \mathcal{M}_{1}(X)$ and $\epsilon>0$

$$
\operatorname{Prob}\left(\frac{1}{N} \sum_{i=1}^{N} \delta_{x_{i}} \in B_{\epsilon}(\mu)\right):=Z_{N, \beta}^{-1} \int_{\delta_{N}^{-1}\left(B_{\epsilon}(\mu)\right)} e^{-\beta N E^{(N)}} d V^{\otimes N}
$$

Hence, formally, as $N \rightarrow \infty$ and $\epsilon \rightarrow 0$, we can take out the factor $e^{-\beta N E^{(N)}}$ to get

$$
\int_{\delta_{N}^{-1}\left(B_{\epsilon}(\mu)\right)} e^{-\beta N E^{(N)}} d V^{\otimes N} \sim e^{-\beta N E(\mu)} \int_{\delta_{N}^{-1}\left(B_{\epsilon}(\mu)\right)} d V^{\otimes N}
$$

Applying the Sanov's LDP result 4.10 to the integral thus suggests that the non-normalized measure

satisfies a LDP with rate functional

$$
\left(\delta_{N}\right)_{*}\left(e^{-\beta H^{(N)}} d V^{\otimes N}\right)
$$

$$
F_{\beta}(\mu):=E(\mu)+\beta^{-1} D_{d V}(\mu)
$$

In order to make this argument rigorous two issues need to be confronted. First, the nature of the convergence in the "Mean Field Approximation" 4.12 has to be specified. Secondly, appropriate conditions on $H^{(N)}$ need to be introduced, ensuring that the "taking out" argument 4.13 is justified. The simplest way to handle both issues is to assume the following "regularity property":

$$
\lim _{N \rightarrow \infty} \sup _{X^{N}}\left|E^{(N)}\left(x_{1}, \ldots x_{N}\right)-E\left(\delta_{N}\right)\right|=0
$$


for a continuous functional $E(\mu)$ on $\mathcal{M}_{1}(X)$ 60]. However, this fails as soon as $H^{(N)}$ is singular, i.e. not point-wise bounded. For example, this happens in the classical "linear" setting of pair-interactions with a mean field scaling:

$$
E^{(N)}\left(x_{1}, \ldots, x_{N}\right)=\frac{1}{N(N-1)} \frac{1}{2} \sum_{i \neq j} g\left(x_{i}, x_{j}\right)
$$

as soon as $g\left(x_{i}, x_{j}\right)$ is singular on the diagonal (as in the case of the complex plane, formula 2.4). Here linearity refers to fact that the differential $d E_{\mid \mu}$ of the corresponding functional $E$ is linear with respect to $\mu$ :

$$
E(\mu)=\frac{1}{2} \int_{X} g(x, y) \mu(y) \otimes \mu(y)
$$

and

$$
d E_{\mid \mu}=-\psi_{\mu}(x):=-\int_{X} g(x, y) \mu(y)
$$

Still, using a different variational method introduced in [92, based on Gibbs variational principle, it follows from [56, 20, 67] that the LDP in question does hold, if $g$ is assumed lower-semi continuous (or, more generally, if an appropriate exponential integrability assumption on $g$ is assumed, as shown in [20], building on [42, 77]).

For a general Hamiltonian it was as shown in 20] that, under certain technical assumptions, the LDP holds if the "Mean Field Approximation" assumption 4.12 is taken to hold in the following sense:

$$
\lim _{N \rightarrow \infty} \int_{X^{N}} E^{(N)} \mu^{\otimes N}=E(\mu)
$$

(which is trivially the case in the "linear case" 4.15). However, in the present setting the Hamiltonian $H^{(N)}$ (as defined by formula 2.21) is both singular and highly non-linear. Moreover, the convergence 4.16 is yet to be established (see Section ??). On the other hand, the "Mean Field Approximation" 4.12 does hold in the sense of Gamma-convergence. Exploiting that $H^{(N)}$ is uniformly super-harmonic, the "taking out" argument in formula 4.13 can then be rigorously justified (see Section 5).

Remark 4.16. The regularity property 4.14 is preserved when the sign of $H^{(N)}$ (or, equivalently, the sign of $\beta$ ) is switched. However, in the present singular setting the sign is crucial and the "negative temperature case" is yet to be established (see Section 7.4).

4.4.1. Mean field equations. Assume that $\mu$ is a critical point of the free energy functional $F_{\beta}$ in the interior of $\mathcal{M}_{1}(X)$ and that the variational derivative of $E(\mu)$ exists at $\mu$, in a suitable sense:

$$
d E_{\mid \mu}=-\varphi_{\mu},
$$

for a function $\varphi_{\mu}$ on $X$, defined up to an additive constant. Then $\mu$ satisfies the following equation, known as a mean field equation in the physics literature,

$$
\mu=e^{\beta \varphi_{\mu}} / Z
$$

for some positive constant $Z$. This follows directly from the fact that the differential of $D_{\mu_{0}}$ at $\mu$ is represented by the function $\log \left(\mu / \mu_{0}\right)$ if $D_{\mu_{0}}(\mu)<\infty$. However, if $E$ is singular and non-linear it is, in general, highly non-trivial to prove that a general minimizer of $E$ satisfies 
the equation 4.17. In the present complex geometric geometric this was accomplished in [15], building on [28].

\section{Proofs for the Limit $N \rightarrow \infty$}

We now turn to the proofs of the results stated in Section 2. We will use the compactification of $\mathbb{C}^{n}$ by $\mathbb{P}^{n}$ to reduce the proofs to the setting of a compact polarized manifold.

Let thus $(X, L)$ be a polarized compact complex manifold and set

$$
N_{k}:=\operatorname{dim} H^{0}(X, k L)=V k^{n}+o\left(k^{n}\right),
$$

where by assumption $V>0$ (see Section 3.2). We fix once and for all the back-ground data $(\|\cdot\|, d V)$ consisting of a Hermitian metric $\|\cdot\|$ on $L$ and a volume form $d V$ on $X$. We will denote by $\omega$ the curvature two-form of the metric $\|\cdot\|$ on $L$. The data $(\|\cdot\|, d V)$ induces a Hilbert space structure on $H^{0}(X, k L)$. The corresponding Slater determinant $\operatorname{det} \Psi^{(k)}$ is the holomorphic section of $(k L)^{\bigotimes N_{k}} \rightarrow X^{N_{k}}$ determined, up to a choice of sign, by demanding that it be totally anti-symmetric and normalized:

$$
\left(N_{k} !\right)^{-1} \int_{X^{N_{k}}}\left\|\operatorname{det} \Psi^{(k)}\right\|^{2} d V^{\otimes N_{k}}=1
$$

Concretely,

$$
\operatorname{det} \Psi^{(k)}\left(x_{1}, \ldots, x_{N_{k}}\right):=\operatorname{det}\left(\Psi_{i}^{(k)}\left(x_{j}\right)\right)_{i, j \leq N_{k}}
$$

where $\Psi_{1}^{(k)}, \ldots, \Psi_{N_{k}}^{(k)}$ is a fixed orthonormal base in the Hilbert space $H^{0}(X, k L)$.

Remark 5.1. The section $\operatorname{det} \Psi^{(k)}$ is uniquely determined, up to a multiplicative non-zero complex number, by the property that it generates the top exterior power $\Lambda^{N_{k}} H^{0}(X, k L)$ of the complex vector space $H^{0}(X, k L)$, viewed as a one-dimensional subspace of $H^{0}\left(X^{N_{k}},(k L)^{\bigotimes N_{k}}\right)$ (the totally anti-symmetric part). In particular, if $\operatorname{det} \widetilde{\Psi}^{(k)}\left(x_{1}, \ldots, x_{N_{k}}\right)$ denotes any other generator, then

$$
\operatorname{det} \widetilde{\Psi}^{(k)}\left(x_{1}, \ldots, x_{N_{k}}\right)=C_{k} \operatorname{det} \Psi^{(k)}\left(x_{1}, \ldots, x_{N_{k}}\right), \quad C_{k} \in \mathbb{C}^{*}
$$

(hence, $\left|C_{k}\right|=1$ if the normalization condition 5.1 holds).

Example 5.2. By homogenization (see Section 3.7) the Vandermonde determinant $D^{\left(N_{k}\right)}$ on $\left(\mathbb{C}^{n}\right)^{N_{k}}$ may be identified with a generator for $\Lambda^{N_{k}} H^{0}\left(\mathbb{P}^{n}, \mathcal{O}(k)\right)$ that we shall denote by the same symbol $D^{\left(N_{k}\right)}$. Concretely, over $\mathbb{P}^{n} D^{\left(N_{k}\right)}$ is of the form 5.2 with $\Psi_{i}^{(k)}$ a basis of homogeneous multinomials of degree $k$. However, this basis is not orthonormal with respect to $(\|\cdot\|, d V)$, for any volume form on $\mathbb{P}^{n}$.

We define the energy $E_{\omega}^{(N)}\left(x_{1}, \ldots, x_{N_{k}}\right)$ of a configuration $x_{1}, \ldots x_{N}$ of $N$ points on $X^{N}$, relative $\omega$, by

$$
E_{\omega}^{(N)}\left(x_{1}, \ldots, x_{N_{k}}\right):=-\frac{1}{N_{k} k} \log \left\|\operatorname{det} \Psi^{(k)}\left(x_{1}, \ldots, x_{N_{k}}\right)\right\|^{2},
$$

which is canonically attached to the data $(X, L,\|\cdot\|, d V)$ or more precisely to $(X, L, \omega, d V)$ (since $\operatorname{det} \Psi^{(k)}$ is uniquely determined up to a sign). 
To the data $(\|\cdot\|, d V, \beta)$, where $\beta$ is a given positive number, we associate the following symmetric probability measure on $X^{N_{k}}$ :

$$
\mu_{\beta}^{\left(N_{k}\right)}:=\frac{\left\|\operatorname{det} \Psi^{(k)}\left(x_{1}, \ldots, x_{N_{k}}\right)\right\|^{2 \beta / k}}{Z_{N_{k}, \beta}} d V^{\otimes N_{k}} .
$$

The corresponding random point process on $X$ will be called the temperature deformed determinantal point process attached to $(\|\cdot\|, d V, \beta)$. The probability measure 5.3 is the Gibbs measure on $X^{N_{k}}$, at inverse temperature $\beta$, associated to the Hamiltonian $N E_{\omega}^{(N)}$ :

$$
\mu_{\beta}^{(N)}:=\frac{e^{-\beta N E_{\omega}^{(N)}}}{Z_{N, \beta}} d V^{\otimes N}
$$

By homogenity, $\mu_{\beta}^{(N)}$ is independent of the choice of generator of $\Lambda^{N_{k}} H^{0}(X, k L)$ (compare Remark 5.1). But the point of choosing the particular generator $\operatorname{det} \Psi^{(k)}$ is that the corresponding determinantal energy $E_{\omega}^{(N)}$ admits a large $N$-limit. This is made precise by the following result (the definition of the projection operator $P_{(K, \omega)}$ is given in Section 3.5):

Theorem 5.3. Let $(X, L)$ be a compact polarized manifold. Then $E_{\omega}^{(N)}$ Gamma-converges towards the pluricomplex energy $E_{\omega}$ on $\mathcal{M}_{1}(X)$, as $N \rightarrow \infty$. As a consequence, if $\left(x_{1}, \ldots, x_{N_{k}}\right)$ is a minimizer $E_{\omega}^{(N)}$ on $X^{N}$, i.e. it maximizes $\left\|\operatorname{det} \Psi^{(k)}\right\|$, then the corresponding empirical measures

$$
\delta_{N}:=N^{-1} \sum_{i=1}^{N} \delta_{x_{i}}
$$

converge weakly towards the unique minimizer $\mu_{\omega}$ of $E_{\omega}^{(N)}$ on $\mathcal{P}(X)$. More generally, if $K$ is a non-polar subset of $X$ and $\left(x_{1}, \ldots, x_{N_{k}}\right)$ is a minimizer $E_{\omega}^{(N)}$ on $K^{N}$, then the corresponding empirical measures converge weakly towards the unique minimizer $\mu_{(K, \omega)}$ of $E_{\omega}$ on $\mathcal{M}_{1}(X)$, where

$$
\mu_{(K, \omega)}=M A_{\omega} \varphi_{(K, \omega)}, \quad \varphi_{(K, \omega)}:=P_{(K, \omega)} 0
$$

Proof. This result follows from combining the general convergence criterion Prop 4.3 with Theorem $\mathrm{A}$ and $\mathrm{B}$ in [26]. To see this denote by $f_{N}$ the functional on $C(X)$ defined as the Legendre-Fenchel transform of $E_{\omega}^{(N)}$ :

$$
f_{N}(u):=\sup _{X^{N}}-\frac{1}{N_{k} k} \log \left\|\operatorname{det} \Psi^{(k)}\left(x_{1}, \ldots, x_{N_{k}}\right)\right\|_{k u}^{2}
$$

where $\|\cdot\|_{k u}$ denotes the metric on $k L$ defined by $\|\cdot\|_{k u}^{2}=\|\cdot\|^{2} e^{-k u}$ (as in Section 3.2). By [26, Thm A]

$$
f_{N}(u) \rightarrow f(u),-f(-u)=\mathcal{E} \circ P_{\omega},
$$

Moreover, by [26, Thm B], the functional $\mathcal{E}\left(P_{\omega} u\right)$ is Gateaux differentiable on $C(X)$. Hence, all that remains is to verify that the Legendre-Fenchel transform of $f$ coincides with $E_{\omega}(\mu)$. But this follows readily from the definition 3.13 of $E_{\omega}(\mu)$, only using that $P_{\omega}$ is an increasing projection from $C(X)$ onto $P S H(X, \omega) \cap C(X)$. Moreover, by general convex duality theory 
(see [15]) it also follows that $E_{\omega}(\mu)$ admits a unique minimizer of $\mathcal{M}_{1}(X)$, namely the differential of $\mathcal{E} \circ P_{\omega}$ at $u=0$, which is given by formula 5.4 (by [26, Thm B]). The latter formula holds as long as $K$ is not pluripolar (which ensures that $P_{(K, \omega)}$ is well-defined).

For completeness, let us also outline the proof of Theorem A in [26], i.e. the convergence 5.6. in the present setting where $L$ is assumed ample. Given $u \in C(X)$ we set

$$
\mathcal{F}_{k, L^{p}}[u]:=-\frac{1}{k N_{k}} \log \left\|\operatorname{det} \Psi^{(k)}\right\|_{L^{p}\left(X^{\left.N_{k}, u, d V\right)}\right.}^{2}
$$

defined in terms of $L^{p}$-norm $\|\cdot\|_{u}$ on $H^{0}\left(X^{N_{k}},(k L)^{N_{k}}\right)$ induced by $(u, d V)$, for $p \in[1, \infty]$ (which is defined to be the ordinary sup-norm for $p=\infty$ and thus independent of $d V$ ). For $p=\infty$, which is the case appearing in the convergence 5.6, first observe that

$$
\mathcal{F}_{k, L^{\infty}}[u]=\mathcal{F}_{k, L^{\infty}}\left[P_{\omega} u\right] .
$$

Indeed, by the definition of the operator $P$ we have

$$
\sup _{X}\left(e^{\varphi-u)}\right)=\sup _{X} e^{\left(\varphi-P_{\omega} u\right)}
$$

for any $\varphi \in P S H(X, \omega)$. Applying the previous equality to each factor of $X^{N_{k}}$ then gives the equality 5.8. Moreover, the functional $\mathcal{F}_{k, L^{\infty}}$ is equicontinuous with respect to the sup-norm on $C(X)$ (as follows from the fact that the $\mathcal{F}_{k, L^{\infty}}$ is increasing and satisfies $\mathcal{F}_{k, L^{\infty}}[u+C]=$ $\mathcal{F}_{k, L^{\infty}}[u]+C$, when $\left.C \in \mathbb{R}\right)$. Accordingly, writing $P_{\omega} u$ as the uniform limit of Kähler potentials $u_{j}$, i.e. smooth $u_{j} \in P S H(X, \omega)$ such that $\omega_{u_{j}}>0$ (using the approximation property in Prop 3.6 ) it is enough to establish the convergence of $\mathcal{F}_{k, L^{\infty}}[\varphi]$ when $\varphi$ is a Kähler potential. To this end one uses that

$$
\mathcal{F}_{k, L^{\infty}}[\varphi]=\mathcal{F}_{k, L^{2}}[\varphi]+o(1)
$$

where the error term $o(1)$ (tending to zero) only depends on the modulus of continuity of $\varphi$. Indeed, this follows directly from applying the standard submean property of holomorphic functions on small coordinate balls on $X$, for each factor of $X^{N_{k}}$. Now, a direct calculation reveals that the differential of $\mathcal{F}_{k, L^{2}}$ at any $u \in C(X)$ is given by

$$
d\left(\mathcal{F}_{k, L^{2}}\right)_{\mid u}=\frac{1}{N_{k}} \rho_{k u} d V
$$

where the function $\rho_{k u}$ is the restriction to the diagonal of the point-wise norm of the Bergman kernel of the Hilbert space $\left(H^{0}(X, k L),\|\cdot\|_{L^{2}(X, u, d V)}\right)$. The asymptotics of $\rho_{k u}$, when $u$ is a Kähler potential, i.e. when it defines a smooth metric on $L$ with strictly positive curvature $\omega_{u}$ are well-known (and due to Bouche and Tian, independently) and, in particular, give that

$$
\text { (i) } \lim _{k \rightarrow \infty} \frac{1}{N_{k}} \rho_{k u} d V=\frac{1}{V}\left(\omega_{u}\right)^{n}(i i) \frac{1}{N_{k}} \rho_{k u} \leq C
$$

in the weak topology (see 12 for an elementary proof in the $\mathbb{C}^{n}$-setting, based on formula 4.11 and the sub-mean property of holomorphic functions). Using the defining property 3.7 of the functional $\mathcal{E}$ (and integrating along a line segment in the space of all positively curved metrics) this gives

$$
\mathcal{F}_{k, L^{\infty}}[u]=\mathcal{E}_{u}(u)+o(1),
$$

which proves the convergence 5.6, thanks to 5.8 and 5.9 . 
Finally, we recall that the differentiability statement (Thm B in [26]) is proved using the "orthogonality relation" (see also [88] for an elegant simplification of the original proof).

Remark 5.4. From a probabilistic point of view, an interesting feature of the previous proof is that it reduces the (deterministic) Gamma-convergence problem to establishing the weak convergence of the the expectations $\mathbb{E}\left(\delta_{N}\right)$ for the corresponding determinantal point process (corresponding to $p=2$ ). Indeed, by formula 4.10, $\mathbb{E}\left(\delta_{N_{k}}\right)$ coincides with the corresponding Bergman measure $\frac{1}{N_{k}} \rho_{k} d V$ and $\mathcal{F}_{k, L^{2}}(u)$ (formula5.7) essentially coincides with the logarithmic moment generating function of the determinantal point process. To make the connection to Kähler geometry one can use formula 5.2 to express the functional $\mathcal{F}_{k, L^{2}}$ as

$$
\mathcal{F}_{k, L^{2}}(u)=-\frac{1}{k N_{k}} \log \left(N_{k} ! \operatorname{det}_{i, j \leq N_{k}}\left\langle\Psi_{i}^{(k)}, \Psi_{j}^{(k)}\right\rangle_{(k u, d V)}\right),
$$

in terms of the scalar product on $H^{0}(X, k L)$ induced by $(u, d V)$. This reveals that $\mathcal{F}_{k, L^{2}}$ is essentially Donaldson's L-functional, which plays a prominent role in Kähler geometry (see [26, 27] and references therein).

We next consider the determinantal energy

$$
E^{\left(N_{k}\right)}\left(z_{1}, \ldots z_{N_{k}}\right):=-\frac{1}{N_{k} k} \log \left|D^{\left(N_{k}\right)}\left(z_{1}, \ldots z_{N_{k}}\right)\right|^{2}
$$

in $\mathbb{C}^{n}$, introduced in connection to the study of Fekete points on compact subsets of $\mathbb{C}^{n}$ in Section 2.2. By the next corollary $E^{\left(N_{k}\right)}$ Gamma-converges towards the (non-weighted) pluricomplex energy $E(\mu)$, defined in Section 3.7.1.

Corollary 5.5. Let $K$ be a compact subset of $\mathbb{C}^{n}$ which is not pluripolar. Then the determinantal energy $E^{\left(N_{k}\right)}$ converges towards the (non-weighted) pluricomplex energy $E(\mu)$ on $\mathcal{M}_{1}(K)$. As a consequence, if $\left(z_{1}, \ldots, z_{N_{k}}\right)$ are minimizers of $E^{\left(N_{k}\right)}$ on $K^{N_{k}}$, i.e. Fekete points for $K$, then the corresponding empirical measure converges weakly towards the pluripotential equilibrium measure of $K$.

Proof. Take a metric $\|\cdot\|$ on $\mathcal{O}(1) \rightarrow \mathbb{P}^{n}$ which coincides with the trivial metric on an open subset containing $K$, under the standard embedding of $\mathbb{C}^{n}$ in $\mathbb{P}^{n}$. Identifying the Vandermonde determinant with a section over $\left(\mathbb{P}^{n}\right)^{N_{k}}$ (using the identifications in Section 3.7), we can, over $K \Subset \mathbb{P}^{n}$, rewrite

$$
\log \left|D^{\left(N_{k}\right)}\right|^{2}=\log \frac{\left\|D^{\left(N_{k}\right)}\right\|^{2}}{\left\|D^{\left(N_{k}\right)}\right\|_{L^{2}\left(X^{N_{k}}\right)}^{2}}+\log \left\|D^{\left(N_{k}\right)}\right\|_{L^{2}\left(X^{N_{k}}\right)}^{2}
$$

By homogenity and Remark 5.1 we may as well replace $D^{\left(N_{k}\right)}$ with $\operatorname{det} \Psi^{(k)}$ in the quotient appearing in the right hand side above. Hence,

$$
-k^{-1} N_{k}^{-1} \log \left|D^{\left(N_{k}\right)}\right|^{2}=E_{\omega}^{(N)}-k^{-1} N_{k}^{-1} \log \left\|D^{\left(N_{k}\right)}\right\|_{L^{2}\left(X^{N_{k}}\right)}^{2}
$$

By the Gamma-convergence in the previous theorem, combined with Prop 4.3, all that remains is to verify that

$$
-\lim _{N_{k} \rightarrow \infty} k^{-1} N_{k}^{-1} \log \left\|D^{\left(N_{k}\right)}\right\|_{L^{2}\left(X^{N_{k}}\right)}^{2}=\mathcal{E}_{\omega}\left(P_{\left.T^{n} 0\right)}\right.
$$


But this follows from [27, Thm A]. Indeed, $D^{\left(N_{k}\right)}$ is the Slater determinant associated to a the basis in $H^{0}\left(\mathbb{P}^{n}, k \mathcal{O}(1)\right)$, which is orthonormal with respect to the weighted measure $(\nu, 0)$, where $\nu$ is the standard invariant measure on the unit $n$-torus $T^{n}$ in $\mathbb{C}^{n} \Subset \mathbb{P}^{n}$ and 0 denotes the trivial metric on $\mathcal{O}(1) \rightarrow \mathbb{C}^{n} \Subset \mathbb{P}^{n}$. This measure is a Bernstein-Markov measure, i.e. the the corresponding Bergman density $\rho_{k}$ has sub-exponential growth. In fact, by the $T^{n}$-symmetry, $\rho_{k}=1$. Hence, 5.11 follows from Theorem [27, Thm A] (the proof for Bernstein-Markov measures is essentially the same as the proof of the case when $\nu=d V$ on $X$, outlined in the proof of the previous theorem, using that 5.9 still holds for a Bernstein-Markov measure).

Turning to the probabilistic setting we next explain the proof of the following general result in [18] (which covers Theorem 2.6, stated in Section 2).

Theorem 5.6. Assume that $X$ is a compact Riemannian manifold without boundary and that the sequence $\frac{1}{N} H^{(N)}\left(x_{1}, \ldots, x_{N}\right)$ Gamma converges to a functional $E(\mu)$ on $\mathcal{M}_{1}(X)$. If moreover, $H^{(N)}$ is uniformly quasi-superharmonic, i.e. there exists a constant $C$ such that for all $N$

$$
\Delta_{x_{i}} H^{(N)}\left(x_{1}, x_{2}, \ldots x_{N}\right) \leq C \quad X^{N}
$$

then the measures

$$
\left(\delta_{N}\right)_{*}\left(e^{-\beta H^{(N)}} d V^{\otimes N}\right)
$$

satisfy a Large Deviation Principle $(L D P)$ on $\mathcal{M}_{1}(X)$ with speed $\beta N$ and rate functional $F_{\beta}(\mu)$. As a consequence, the laws of the random measures $\delta_{N}$ on $\left(X^{N}, \mu_{\beta}^{(N)}\right)$ satisfy a Large Deviation Principle (LDP) with speed $\beta N$ and rate functional $F_{\beta}(\mu)-C_{\beta}$, where

$$
C_{\beta}=\inf _{\mathcal{M}_{1}(X)} F_{\beta}
$$

In particular, if $E$ is convex and $\beta>0$ then $\delta_{N}$ converges in law towards the unique minimizer $\mu_{\beta}$ of $F_{\beta}$.

Proof. The idea of the proof is to make rigorous the formal mean field approximation argument given in Section 4.4, by exploiting the assumed quasi-superharmonicity of $H^{(N)}$. Using the characterization of a LDP in Proposition 4.9, the upper bound in the first LDP stated in the theorem follows almost immediately from the liminf property of the Gamma-convergence together with Sanov's theorem. Indeed,

$$
\int_{\delta_{N}^{-1}\left(B_{\epsilon}(\mu)\right)} e^{-\beta N E^{(N)}} d V^{\otimes N} \leq \sup _{\delta_{N}^{-1}\left(B_{\epsilon}(\mu)\right)} e^{-\beta N E^{(N)}} \int_{\delta_{N}^{-1}\left(B_{\epsilon}(\mu)\right)} d V^{\otimes N},
$$

Hence, using the assumed Gamma-convergence gives

$\limsup _{N \rightarrow \infty} N^{-1} \log \int_{\delta_{N}^{-1}\left(B_{\epsilon}(\mu)\right)} e^{-\beta N E^{(N)}} d V^{\otimes N} \leq-\beta \inf _{B_{\epsilon}(\mu)} E(\mu)+\limsup _{N \rightarrow \infty} N^{-1} \log \int_{\delta_{N}^{-1}\left(B_{\epsilon}(\mu)\right)} d V^{\otimes N}$,

Finally, letting $\epsilon \rightarrow 0$ and invoking Sanov's LDP result 4.10 concludes the proof of the upper bound in the LDP.

In order to prove the lower bound in the LDP we first observe that it is enough to establish an estimate of the following form: there exists a constant $C>0$ and $r(\epsilon)$ tending to zero, as 
$\epsilon \rightarrow 0$, such

$$
e^{C N \epsilon} \int_{\delta_{N}^{-1}\left(B_{\epsilon}\left(\delta_{N}\left(\boldsymbol{x}^{(N)}\right)\right)\right.} e^{-\beta N E^{(N)}} d V^{\otimes N} \geq \sup _{\delta_{N}^{-1}\left(B_{r(\epsilon)}\left(\delta_{N}\left(\boldsymbol{x}^{(N)}\right)\right)\right)} e^{-\beta N E^{(N)}} \int_{\delta_{N}^{-1}\left(B_{r(\epsilon)}(\mu)\right)} d V^{\otimes N},
$$

for any given $\boldsymbol{x}^{(N)}:=\left(x_{1}, . ., x_{N}\right) \in X^{N}$. Indeed, by the Gamma-convergence assumption there exists a sequence $\boldsymbol{x}^{(N)}:=\left(x_{1}, . ., x_{N}\right) \in X^{N}$ such that $\delta_{N}\left(\boldsymbol{x}^{(N)}\right) \rightarrow \mu$ and $E^{(N)}\left(\boldsymbol{x}^{(N)}\right) \rightarrow E(\mu)$. In particular,

$$
\delta_{N}^{-1}\left(B_{\epsilon}(\mu)\right) \supseteq \delta_{N}^{-1}\left(B_{r(\epsilon)}\left(\delta_{N}\left(\boldsymbol{x}^{(N)}\right)\right),\right.
$$

when $N$ is sufficiently large. Hence, if 5.12 holds, then

$$
e^{C N \epsilon} \int_{\delta_{N}^{-1}\left(B_{\epsilon}\left(\delta_{N}\left(\boldsymbol{x}^{(N)}\right)\right)\right.} e^{-\beta N E^{(N)}} d V^{\otimes N} \geq e^{-\beta N E^{(N)}\left(\boldsymbol{x}^{(N)}\right)} \int_{\delta_{N}^{-1}\left(B_{r(\epsilon)}(\mu)\right)} d V^{\otimes N} .
$$

From here the rest argument proceeds essentially as in the proof of the upper bound. The good news is that the estimate 5.12 does hold with $r(\epsilon)=\epsilon^{2}$, when the metric $d$ on $\mathcal{M}_{1}(X)$ is taken as the Wasserstein $L^{2}$-metric $d_{W_{2}}$ induced from the Riemannian metric $g$ on $X$. This is the content of [18, Prop 3.9], which thus concludes the proof of the LDP in question.

Finally, let us point out that the starting point of the proof of the inequality 5.12 is the well-known fact that the embedding $\delta_{N}$ of $X^{N} / S_{N}$ into $\left(\mathcal{M}_{1}(X), d_{W_{2}}\right)$ is an isometry when $X^{N} / S_{N}$ is endowed with the quotient space metric induced from the Riemannian metric $g_{N}$ on $X^{N}$, defined as $N^{-1}$ times the product Riemannian metric. The quasi-subharmonicity assumption on $E^{(N)}$ is equivalent to

$$
\Delta_{g_{N}} E^{(N)} \geq-\lambda
$$

on $X^{N}$. Moreover, the scaling of $g_{N}$ also ensures that the Ricci curvature of $g^{(N)}$ is bounded from below by a uniform constant times the dimension of $X^{N}$. The inequality 5.12 now follows from the general sub-mean inequality in [18, Thm 2.1] for Riemannian quotients (orbifolds) $Y:=M / G$ (which yields a distortion factor with sub-exponential growth in the dimension). In turn, the latter inequality is proved using geometric analysis on the orbifold $Y$, by generalizing a fundamental inequality of Li-Schoen in Riemannian geometry [83.

The previous theorem, applied to the temperature-deformed determinantal point processes [5.3, yields the following LDP obtained from [18]:

Theorem 5.7. Let $(X, L)$ be a compact polarized manifold and consider the temperature deformed determinantal point processes $\left(X^{N}, \mu_{\beta}^{(N)}\right)$ on $X$ associated to given data $(\|\cdot\|, d V, \beta)$ with $\beta>0$. Then the the laws of the random measures $\delta_{N}$ on $\left(X^{N}, \mu_{\beta}^{(N)}\right)$ satisfy a Large Deviation Principle (LDP) with speed $\beta N$ and rate functional $F_{\beta}(\mu)-C_{\beta}$, where

$$
F_{\beta}(\mu)=E_{\omega}(\mu)+\beta^{-1} D_{d V}(\mu)-C_{\beta}
$$

with

$$
C_{\beta}=\inf _{\mathcal{M}_{1}(X)} F_{\beta}
$$

Moreover, $F_{\beta}$ admits a unique minimizer $\mu_{\beta}$ on $\mathcal{M}_{1}(X)$ and it can be expressed as $\mu=e^{\beta u_{\beta}} d V$, where $u_{\beta}$ is the unique finite energy solution to the complex Monge-Ampère equation

$$
M A_{\omega}(u)=e^{\beta u} d V .
$$


More generally, the corresponding statement holds when $d V$ is replaced by a singular finite measure $d V_{s}$ of the form

$$
d V_{s}=e^{v} d V
$$

for $v$ a function on $X$ with analytic singularities, i.e locally $v=u_{0}+v$, where $u_{0}$ is continuous and $v$ has analytic singularities, i.e. locally

$$
v=\sum_{i=1}^{m} \lambda_{i} \log \left|h_{i}\right|^{2}
$$

for some $\lambda_{i} \in \mathbb{R}$ and holomorphic functions $h_{i}$.

Proof. The LDP in the case of a volume form $d V$ follows directly from the previous theorem together with the Gamma-convergence in Theorem 5.3 (the quasi-suberharmonicity of $E^{(N)}$ is a consequence of quasi-plurisubharmonicity in example 3.5). The singular case $d V_{s}$ also follows in a similar way (see [19]). Since $E$ is convex and $S$ is strictly convex $F_{\beta}$ admits precisely one minimizer. Finally, to see that the minimizer satisfies the mean field type equation 5.3 one can use the sub-gradient property in Prop 3.9] and the convexity of $F_{\beta}$ (see [19]).

We next turn to the $\mathbb{C}^{n}$-version of the previous theorem, which is a new result (which covers Theorem 2.7, stated in Section 21). We will denote by $E_{\phi}(\mu)$ the weighted pluricomplex energy of a probability measure $\mu$ on $\mathbb{C}^{n}$, defined in Section 3.7.1.

Theorem 5.8. Given a weighted measure $(\phi, d V)$ which is admissible with respect to $\beta$ (formula 2.23) the random measures $\delta_{N_{k}}$ on the probability spaces $\left(\left(\mathbb{C}^{n}\right)^{N}, \mu_{\beta}^{\left(N_{k}\right)}\right)$ converge in law , as $N_{k} \rightarrow \infty$, to the unique minimizer $\mu_{\beta}$ of the following free energy type functional on $\mathcal{M}_{1}\left(\mathbb{C}^{n}\right)$ :

$$
F_{\phi, \beta}(\mu):=E_{\phi}(\mu)+\beta^{-1} D_{d V}(\mu),
$$

Moreover, the minimizer satisfies $\mu=e^{\beta \psi_{\beta}} d V$, where $\psi_{\beta}$ is the unique solution in $\mathcal{E}^{1}\left(\mathbb{C}^{n}\right)$ to

$$
M A(\psi)=e^{\beta(\psi-\phi)} d V
$$

Proof. We will identify $\phi$ with a lsc on $X:=\mathbb{P}^{n}$ which is equal to $\infty$ on $A:=\mathbb{P}^{n}-\mathbb{C}^{n}$. Accordingly, we identify the probability measure $\mu_{\beta}^{(N)}$ on $\left(\mathbb{C}^{n}\right)^{N_{k}}$ with a probability measure on $X^{N_{k}}$. We are going to prove the stronger result that the laws of the corresponding empirical measure $\delta_{N}$ satisfy an LDP with rate functional $F_{\phi, \beta}$ on $\mathcal{M}_{1}(X)$. To this end, first recall that we may assume that $d V=d \lambda$ so that the growth condition becomes

$$
\phi \geq(1+n / \beta+\epsilon) \phi_{F S}-C
$$

on $\mathbb{C}^{n}$ (as pointed out in the discussion before the statement of Theorem 2.7)

Step 1: The LDP holds in the case when $\phi(z)=C_{\beta} \phi_{F S}+u$ for some constant $C_{\beta}$ and function $u$ such that $u$ extends to a continuous function on $\mathbb{P}^{n}$.

In this case we can rewrite

$$
e^{-\beta \phi} d V=e^{-\left(\beta \phi_{F S}+u\right)} d V_{s}, \quad d V_{s}:=e^{-v} d V_{F S}, \quad v(z)=C^{\prime} \phi_{F S}(z)
$$

The LDP now follows from the previous theorem, using that $v$ has analytic singularities, viewed as a function on $\mathbb{P}^{n}$. Indeed, expressed in terms of homogeneous coordinates $Z_{i}$ on $\mathbb{P}^{n}$, viewed as holomorphic sections of $\mathcal{O}(1) \rightarrow \mathbb{P}^{n}$, we have have $\phi_{F S}:=\log \left(1+|z|^{2}\right)=$ $\log \left(\left|Z_{0}\right|^{2}+\ldots+\left|Z_{n}\right|^{2}\right)$ on $\mathbb{P}^{n}$ which is of the form 5.14.

Step 2: The LDP holds when $\phi$ has at most polynomial growth 
Given a weight function $\phi$ we will denote by $Z_{N}[\phi]$ the corresponding partition function (we shall drop the subscript $\beta$ in the following). It will be enough (and, in fact, equivalent) to prove that the following holds for all admissible weight functions:

$$
-\lim _{N \rightarrow \infty} \beta^{-1} N^{-1} \log Z_{N}[\phi]=\inf _{\mathcal{M}_{1}(X)} F_{\phi}
$$

Indeed, applying the formula to $\phi+u$ for any $u \in C(X)$ the LDP then follows from the Gärtner-Ellis theorem (as explained in [18, Section 4.2]). To this end let us first prove the following bound, only assuming that $\phi$ is lsc on $\mathbb{C}^{n}$ and that the growth assumption 2.23 holds:

$$
\inf _{\mathcal{M}_{1}(X)} F_{\phi} \leq \liminf _{N \rightarrow \infty}-\beta^{-1} N^{-1} \log Z_{N}[\phi]
$$

First observe that there exists a sequence $\phi_{j}$, satisfying the assumptions in Step one and increasing to $\phi$. Indeed, taking $C_{\beta}:=(1+n / \beta+\epsilon)$ we have that $\phi(z)=C_{\beta} \phi_{F S}+u$, where $u$ is a lsc continuous function on $\mathbb{C}^{n}$ which is bounded from below. Hence, $u$ extends to a lsc function on $\mathbb{P}^{n}$. This means that there exists a sequence of functions $u_{j} \in C\left(\mathbb{P}^{n}\right)$ such that $u_{j}$ increases to $u$ on $\mathbb{P}^{n}$ and we can thus take $\phi_{j}:=C_{\beta} \phi_{F S}+u_{j}$. Now, since, $\phi_{j} \leq \phi$ we have, for any $j$ and $N$,

$$
-\beta^{-1} N^{-1} \log Z_{N}\left[\phi_{j}\right] \leq-\beta^{-1} N^{-1} \log Z_{N}[\phi]
$$

Using the LDP in Step one thus gives

$$
\inf _{\mathcal{M}_{1}(X)} F_{\phi_{j}} \leq \liminf _{N \rightarrow \infty}-\beta^{-1} N^{-1} \log Z_{N}[\phi]
$$

Finally, letting $j \rightarrow \infty$ proves the lower bound 5.16 .

To prove the upper bound we will also use the assumption that $\phi$ has at most polynomial growth. Fix $R>0$ and set

$$
\phi_{R}:=\min \left\{\phi, R \phi_{F S}\right\}, K_{R}:=\left\{\phi=\phi_{R}\right\}
$$

We may assume that the increasing compact subsets $K_{R}$ exhaust $\mathbb{C}^{n}$ as $R \rightarrow \infty$ (otherwise we are in the realm of the previous step). Denote by $\mu_{R}^{(N)}$ the Gibbs measure with Hamiltonian $H_{\phi_{R}}^{(N)}$. By Jensen's inequality

$$
-\beta^{-1} N^{-1} \log Z_{N}[\phi] \leq-\beta^{-1} N^{-1} \log Z_{N}\left[\phi_{R}\right]+N^{-1} \sum_{i=1}^{N} \int\left(\phi-\phi_{R}\right)\left(z_{i}\right)\left(\mu_{R}^{(N)}\right)
$$

Next, we note that, by permutation symmetry,

$$
N^{-1} \sum_{i=1}^{N} \int\left(\phi-\phi_{R}\right)\left(z_{i}\right)\left(\mu_{R}^{(N)}\right)=\int_{\mathbb{C}^{n}}\left(\phi-\phi_{R}\right)\left(\mu_{R}^{(N)}\right)_{1},
$$

where $\left(\mu_{R}^{(N)}\right)_{1}$ denotes the one-point correlation measure of $\mu_{R}^{(N)}$ (see Remark 4.6). Thus, in order to prove the upper bound in 5.15, it will now be enough to prove

$$
\limsup _{R \rightarrow \infty} \limsup _{N \rightarrow \infty} \int_{\mathbb{C}^{n}}\left(\phi-\phi_{R}\right)\left(\mu_{R}^{(N)}\right)_{1}=0
$$

To this end it is, in turn, enough to prove the following

$$
\text { claim: }\left(\mu_{R}^{(N)}\right)_{1} \leq C e^{-\phi_{R-1}} d V_{s}
$$


for a constant $C$, independent of $R$ and $N$. Indeed, then the integral in formula 5.18 may be estimated from above as follows, for $R$ sufficiently large,

$$
\int_{K_{R}} \phi\left(\mu_{R}^{(N)}\right)_{1} \leq C \int_{K_{R}} \phi e^{-\phi_{R-1}} d V_{s} \leq \epsilon_{R}
$$

where $\epsilon_{R}$ is independent of $N$ and tends to zero as $R \rightarrow \infty$. In the last inequality we used that $\phi$ is assumed to have at most polynomial growth and hence there exists a function $g \in L^{1}\left(\mathbb{C}^{n}, d V_{s}\right)$ such that $\phi e^{-\phi_{R-1}} \leq g$ for any sufficiently large $R$.

All that remains is thus to prove the claim 5.19. To this end first observe that, by definition,

$$
\left(\mu_{R}^{(N)}\right)_{1}(z):=d V_{s}(z) \frac{1}{Z_{N}\left[\phi_{R}\right]} \int_{\mathbb{C}^{N-1}} e^{-\beta H_{\phi_{R}}^{(N)}\left(z, z_{2}, \ldots, z_{N}\right)} d V_{s}\left(z_{2}\right) \cdots d V_{s}\left(z_{N}\right)
$$

Since $-H^{(N)}\left(\cdot, z_{2}, \ldots, z_{N}\right)$ is in $\mathcal{L}\left(\mathbb{C}^{n}\right)$ it follows that

$$
\left(\mu_{R}^{(N)}\right)_{1}(z) \leq d V_{s}(z) \sup _{\psi \in \mathcal{L}\left(\mathbb{C}^{n}\right)} \frac{e^{\left(\psi-\phi_{R}\right)(z)}}{\int_{\mathbb{C}^{n}} e^{\left(\psi-\phi_{R}\right)} d V_{s}}
$$

To proof of the claim 5.19 is thus reduced to the prove of the following

$$
\text { claim: } f_{R}(z):=\sup _{\psi \in \mathcal{L}\left(\mathbb{C}^{n}\right)} \frac{e^{\left(\psi-\phi_{F S}(z)\right)}}{\int_{\mathbb{C}^{n}} e^{\left(\psi-\phi_{R}\right)} d V_{s}} \leq C
$$

for some constant $C$, independent of $R$. To prove the latter claim first rewrite

$$
\frac{e^{\left(\psi-\phi_{F S}(z)\right)}}{\int_{\mathbb{C}^{n}} e^{\left(\psi-\phi_{R}\right)} d V_{s}}=\frac{e^{\varphi(z)}}{\int_{\mathbb{C}^{n}} e^{\varphi_{\nu}}}, \nu_{R}:=e^{\phi_{F S}-\phi_{R}} d V_{s}
$$

Since $\nu_{R}$ decreases to $\nu:=e^{\phi_{F S}-\phi} d V_{s}$ as $R \rightarrow \infty$ we get, by identifying $\nu$ with a measure on $\mathbb{P}^{n}$,

$$
f_{R}(z) \leq \sup _{P S H\left(\mathbb{P}^{n}, \omega_{F S}\right)} \frac{e^{\varphi(z)}}{\int_{\mathbb{P}^{n}} e^{\varphi} \nu}=\sup _{P S H\left(\mathbb{P}^{n}, \omega_{F S}\right)_{0}} \frac{1}{\int_{\mathbb{P}^{n}} e^{\varphi} \nu},
$$

where $P S H\left(\mathbb{P}^{n}, \omega_{F S}\right)_{0}$ denotes the space of all $\omega_{F S}-$ psh $\varphi$ such that $\sup _{\mathbb{P} n} \varphi=0$. By Prop 3.6 the latter space is compact in $L^{1}\left(\mathbb{P}^{n}\right)$. The proof of the claim 5.21 is thus concluded by noting that the functional $\int_{\mathbb{P}^{n}} e^{e} \nu$ is continuous wrt the $L^{1}$-topology on $P S H\left(\mathbb{P}^{n}, \omega_{F S}\right)$ (indeed, if $\varphi_{j} \rightarrow \varphi$ in $L^{1}$ then $\varphi_{j} \leq C$ and, after perhaps passing to a subsequence, $\varphi_{j} \rightarrow \varphi$ a.e. so that the dominated convergence theorem can be invoked).

Step 3: The case of iterated exponential growth

First assume that $\phi$ has exponential growth. Take $\phi_{R}$ increasing to $\phi$, as $R \rightarrow \infty$ and such that $\phi_{R}$ is continuous and has polynomial growth. By Step 2, the asymptotics 5.15 hold for the weight functions $\phi_{R}$. Now, since, $\phi$ has exponential growth there exists a function $g \in L^{1}\left(\mathbb{C}^{n}, d V_{s}\right)$ such that $\phi e^{-\phi_{R}} \leq g$ for any sufficiently large $R$. Hence, we can repeat the argument in the proof of Step 2 to conclude that the LDP also holds when $\phi$ has exponential growth. Iterating this argument conclude the proof of Step 3 and hence of the LDP on $\mathbb{P}^{n}$.

Remark 5.9. The LDP on $\mathcal{M}_{1}\left(\mathbb{P}^{n}\right)$ implies, in fact, that the LDP also holds on $\mathcal{M}_{1}\left(\mathbb{C}^{n}\right)$ (defined as the topological dual of the space of bounded functions on $\mathbb{C}^{n}$ ) with a good rate functional obtained from the embedding of $\mathbb{C}^{n}$ into $\mathbb{P}^{n}$ (thus coinciding with $F_{\beta, \phi}$ ). This follows from general principles (the contraction principle), only using that $\mathbb{C}^{n}=X-A$ where $A$ is pluripolar. 


\section{Proofs For the Limits $\beta \rightarrow \infty$ AND $\beta \rightarrow 0$}

In this section the line bundle $L$ plays no role. Accordingly, we will consider the more general "transcendental" setting of a compact complex manifold $X$ endowed with a real closed $(1,1)$ - form $\omega$, assumed cohomologous to a Kähler form on $X$ (see Section 3.3).

For any given measure $\mu_{0}$ on $X$, not charging pluripolar subsets, there exists [15, Thm 3.2] a unique $\varphi_{\beta} \in \mathcal{E}^{1}(X, \omega)$ such that

$$
M A_{\omega}\left(\varphi_{\beta}\right)=e^{\beta \varphi_{\beta}} \mu_{0}
$$

when $\beta>0$ (however, when $\beta=0$ one needs to assume that $E_{\omega}\left(\mu_{0}\right)<\infty$; see Theorem 3.8).

Following [28] we recall that a sequence $\varphi_{j} \in P S H(X, \omega)$ is said to converge to $\varphi_{\infty}$ in energy, if the convergence holds in $L^{1}(X)$ and $\mathcal{E}\left(\varphi_{j}\right)$ converges to $\mathcal{E}(\varphi)$, which moreover is assumed finite.

6.1. The limit $\beta \rightarrow \infty$. We start by considering the "zero temperature-limit" where $\beta \rightarrow \infty$. Assume that $\mu_{0}$ is supported on a compact subset $K$ of $X$. We will say that that $\mu_{0}$ is determining wrt $(K, \omega)$ if

$$
\varphi \leq 0 \text { almost everywhere wrt } \mu_{0} \Longrightarrow \varphi \leq 0 \text { on } K
$$

Theorem 6.1. Let $X$ be a compact complex manifold and $\mu_{0}$ a measure on $X$ with support $K$ such that $\mu_{0}$ does not charge pluripolar subsets and which is determining for $(K, \omega)$. Then $\varphi_{\beta}$ converges to $\varphi_{(K, \omega)}$ in energy, as $\beta \rightarrow \infty$. If moreover, $\mu_{0}$ is determining for $\left(K, \omega_{u}\right)$, for any $u \in C(X)$, then

$$
F_{\beta}:=E_{\omega}+\beta^{-1} D_{\mu_{0}}
$$

Gamma-converges towards $E_{\omega}$, as $\beta \rightarrow \infty$.

Proof. The first part is proved essentially as in [21], where it is assumed that $K=X$. The proof may be summarized as follows. First, by a concavity argument, $\varphi_{\beta}$ is a minimizer of the functional

$$
\mathcal{J}_{\beta}(\varphi):=-\mathcal{E}(\varphi)+\mathcal{L}_{\beta}(\varphi), \quad \mathcal{L}_{\beta}(\varphi):=\beta^{-1} \log \int e^{\beta \varphi} \mu_{0}
$$

on $\mathcal{E}^{1}(X, \omega)$. In fact, the existence of the solution $\varphi_{\beta}$ can be shown by minimizing $\mathcal{J}_{\beta}$ [15, Thm 3.2]. Now, by the determining property and [27, Thm 1.14] there exists a constant $C$ such that

$$
\sup _{K} \varphi-C / \beta-\epsilon \leq \mathcal{L}_{\beta}(\varphi) \leq \sup _{K} \varphi+C / \beta
$$

Moreover, the functional $\sup _{K}(\cdot)$ is continuous on $\operatorname{PSH}(X, \omega)$ [27, Cor 1.16]. Since,

$$
\mathcal{L}_{\beta}\left(\varphi_{\beta}\right)=0
$$

a compactness argument allows one to conclude that, after perhaps passing to a subsequence, $\varphi_{\beta}$ converges in $L^{1}$ to a minimizer of

$$
\mathcal{J}_{\infty}(\varphi):=-\mathcal{E}(\varphi)+\sup _{K} \varphi
$$

on the subspace $\mathcal{K}$ of $P S H(X, \omega)$ consisting of all $\varphi$ such that $\sup _{K} \varphi=0$. Next, one shows that there exists a unique minimizer, namely $\varphi=P_{(K, \omega)} 0$ (using that $\mathcal{E}$ is strictly increasing 
on the space of finite energy functions in $P S H(X, \omega))$. Hence, $\varphi_{\beta} \rightarrow P_{(K, \omega)} 0$ in $L^{1}$ and

$$
\inf _{P S H(X, \omega)} \mathcal{J}_{\beta} \rightarrow-\mathcal{E}\left(P_{K} 0\right)
$$

Using 6.3 this implies that $\mathcal{E}\left(\varphi_{\beta}\right) \rightarrow \mathcal{E}\left(P_{(K, \omega)} 0\right)$, i.e the convergence holds in energy, as desired.

To prove the Gamma-convergence of $F_{\beta}$ we first observe that

$$
\inf _{P S H(X, \omega)} \mathcal{J}_{\beta}=\inf _{\mathcal{M}_{1}(K)} E_{\omega}+\beta^{-1} D_{\mu_{0}}
$$

This can be checked explicitly, but it also follows from general duality considerations (see [15]).

Now, replacing $\omega$ with $\omega_{u}$ we deduce from 6.4 that

$$
\inf _{P S H(X, \omega)} \mathcal{J}_{\beta, u} \rightarrow-\mathcal{E}\left(P_{K} u\right)
$$

where $\mathcal{J}_{\beta, u}$ is defined by replacing $\mu_{0}$ in the definition of $\mathcal{L}_{\beta}$ with $e^{-\beta u} \mu_{0}$. Moreover, by 6.5

$$
-\inf _{P S H(X, \omega)} \mathcal{J}_{\beta, u}=\inf _{\mathcal{M}_{1}(K)}\left(E_{\beta}(\mu)+\beta^{-1} D_{\mu_{0}}(\mu)+\int u \mu\right)
$$

(using that $D_{e^{-\beta u} \mu_{0}}=D_{\mu_{0}}+\beta \int u \mu$ ). Now, combining 6.7 and 6.6 reveals that the LegendreFenchel transform on $C(K)$ of $F_{\beta}$ converges towards the functional $-\mathcal{E} \circ P_{(K, \omega}(-\cdot)$, which is Gateaux differentiable [26, Thm B] (see the proof of Theorem 5.3). Hence, the Gammaconvergence in question follows from Prop 4.3, also using that $E_{\omega}$ is the Legendre-Fenchel transform of $-\mathcal{E} \circ P_{(K, \omega)}(-\cdot)$ (just as in the proof of Theorem 5.3 ).

The previous theorem immediately implies Theorem 2.12 in $\mathbb{C}^{n}$, using the identifications in Section 3.7 .

6.2. The limit $\beta \rightarrow 0$. Next, we will consider the "infinite temperature limit" when $\beta \rightarrow 0$.

Theorem 6.2. Let $X$ be a compact complex manifold and $\mu_{0}$ a probability measure on $X$ such that $E_{\omega}(\mu)<\infty$. When $\beta \rightarrow 0$ the functions $\varphi_{\beta}$ converge to $\varphi_{0}$ in energy, where $\varphi_{0}$ is the unique solution to

$$
M A_{\omega}(\varphi)=\mu_{0}
$$

satisfying the normalization condition

$$
\int_{X} \varphi \mu_{0}=0
$$

More generally, given a lsc function $u_{0}$ on $X$ we consider the equation 6.1 with $\mu_{0}$ replaced by $e^{-\beta u_{0}} \mu_{0}$. Then the result above still holds under the assumption that

$$
E_{\omega, u_{0}}(\mu):=E_{\omega}(\mu)+\int u_{0} \mu<\infty
$$

if $\varphi$ is replaced by $\varphi-u_{0}$ in the normalization condition 6.8.

Proof. The function $\varphi_{\beta}$ is the unique minimizer of the functional $\mathcal{J}_{\beta}$ appearing in the beginning of the proof of Theorem 6.1, subject to the normalization condition

$$
\mathcal{L}_{\beta}(u)=0
$$

Setting $\mathcal{L}_{0}(u):=\int u \mu_{0}$ we thus need to prove that $u_{\beta}$ converges towards the unique minimizer $u_{0}$ of $\mathcal{J}_{0}$, subject to the normalization condition 6.9. 
Step 1: After perhaps passing to a subsequence $\varphi_{\beta}$ converges to $\varphi_{0}$ where $\varphi_{0}$ minimizes $\mathcal{J}_{0}$ and moreover

$$
\mathcal{J}_{\beta}\left(\varphi_{\beta}\right) \rightarrow-E_{\omega}\left(\mu_{0}\right)
$$

To prove this first observe that $\mathcal{L}_{0}(u) \leq \mathcal{L}_{\beta}(u)$ for any $\beta>0$ and function $u$. Hence, for any given $\varphi \in P S H(X, \omega) \cap C(X)$ we have, using the very definition of $E_{\omega}\left(\mu_{0}\right)$, that

$$
-E_{\omega}\left(\mu_{0}\right) \leq \mathcal{J}_{0}\left(\varphi_{\beta}\right) \leq \mathcal{J}_{\beta}\left(\varphi_{\beta}\right) \leq \mathcal{J}_{\beta}(\varphi) \leq \mathcal{J}_{0}(\varphi)+C_{\varphi} \beta
$$

In particular, using the normalization condition 6.9 we deduce that

$$
-E_{\omega}\left(\mu_{0}\right) \leq-\mathcal{E}\left(\varphi_{\beta}\right) \leq C
$$

Next, we recall the following coercivity estimate [28]: for a fixed volume form $d V$ on $X$ there exists positive constant $C$ such that

$$
\mathcal{J}_{0}(\varphi) \geq C\left(-\mathcal{E}(\varphi)+\int \varphi d V\right)-C
$$

on $\operatorname{PSH}(X, \omega)$. Combined with the inequality 6.11 this gives

$$
\int \varphi_{\beta} d V \leq C^{\prime}
$$

By the $L^{1}$-compactness of $P S H(X, \omega)_{0}$ (Prop [3.6) we deduce that, after perhaps passing to a subsequence, $\varphi_{\beta} \rightarrow \varphi_{0}$ in $L^{1}(X)$. Since $\mathcal{J}_{0}$ is lsc the inequalities 6.10 thus imply that $\varphi_{0}$ minimizes $\mathcal{J}_{0}$, as desired. Moreover, it also follows from the inequalities 6.10 that $\mathcal{J}_{\beta}\left(\varphi_{\beta}\right) \rightarrow \mathcal{J}_{0}\left(\varphi_{0}\right)=-E_{\omega}\left(\mu_{0}\right)$. Hence, by the normalization condition $6.9 \mathcal{E}\left(\varphi_{\beta}\right) \rightarrow \mathcal{E}\left(\varphi_{0}\right)$, which concludes the proof of Step 1.

Step 2: $\int \varphi_{\beta} \mu_{0} \rightarrow 0$

First observe that, when $\beta>0$ and $\mu_{\beta}:=M A_{\omega}\left(\varphi_{\beta}\right)$

$$
-\mathcal{J}_{\beta}\left(\varphi_{\beta}\right)=F_{\beta}\left(\mu_{\beta}\right):=E_{\omega}\left(\mu_{\beta}\right)+\beta^{-1} D_{\mu_{0}}\left(\mu_{\beta}\right)
$$

as follows from 6.5. By the previous step the lhs above converges towards $E_{\omega}\left(\mu_{0}\right)$ and so does $E_{\omega}\left(\mu_{\beta}\right)$ (since $\varphi_{\beta}$ converges towards $\varphi_{0}$ in energy). Hence,

$$
\beta^{-1} D_{\mu_{0}}\left(\mu_{\beta}\right) \rightarrow 0 \text {. }
$$

But, since $\mu_{\beta}=e^{\beta \varphi_{\beta}} \mu_{0}$ we have $\beta^{-1} D_{\mu_{0}}\left(\mu_{\beta}\right)=\int \varphi_{\beta} \mu_{\beta}$, which the concludes the proof of Step 2 .

Now, by Step 1, $\varphi_{\beta_{j}} \rightarrow \varphi_{0}$ where $\varphi_{0}$ minimizes $\mathcal{J}_{0}$ and hence solves the equation 3.15 (by Theorem [3.8). Next, by Step 2 and the fact that $\mathcal{L}_{0}$ is continuous wrt convergence in energy [28, we have $\mathcal{L}_{0}\left(\varphi_{0}\right)=0$. Hence, by the uniqueness in Theorem 3.8 the whole family $\varphi_{\beta}$ converges to $\varphi_{0}$, which has the required property.

This concludes the proof of the theorem in the case when $u_{0}=0$. In order to prove the general case we fix a family $u^{R} \in C(X)$ such that $u^{R}$ increases to $u_{0}$ as $R \rightarrow \infty$. The existence of $\varphi_{\beta}$ follows from the previous case, by setting

$$
\mathcal{L}_{\beta}(u):=\beta^{-1} \log \int e^{\beta\left(u-u_{0}\right)} \mu_{0}
$$


and using that the measure $e^{-\beta u_{0}} \mu_{0}$ has finite energy (since it is dominated by $C_{0} \mu_{0}$ ). Moreover, the inequalities 6.10 still hold if $E_{\omega}\left(\mu_{0}\right)$ is replaced by $E_{\omega, u_{0}}(\mu)$ and the last inequality is replaced by

$$
\mathcal{J}_{\beta, u_{0}}(\varphi) \leq-\mathcal{E}(\varphi)+\int\left(\varphi-u^{R}\right) \mu_{0}+C_{u, R} \beta
$$

(only using that $u \geq u^{R}$ ). We note that in the limit when first $\beta \rightarrow 0$ and then $R \rightarrow \infty$ the right hand side above tends to $\mathcal{J}_{0, u_{0}}(\varphi)$. We can then conclude essentially as before.

Corollary 6.3. Let $\mu_{0}$ be a probability measure on $\mathbb{C}^{n}$ and $\phi$ a continuous function on $\mathbb{C}^{n}$ with super logarithmic growth. Assume that $E_{\phi}\left(\mu_{0}\right)<\infty$. Given $\beta>0$ there exists a unique $\psi_{\beta} \in \mathcal{E}^{1}\left(\mathbb{C}^{n}\right)$ such that

$$
M A\left(\psi_{\beta}\right)=e^{\beta\left(\psi_{\beta}-\phi\right)} \mu_{0}
$$

Moreover, when $\beta \rightarrow 0$ the functions $\psi_{\beta}$ converge to $\psi_{0}$ in energy, where $\psi_{0}$ is the unique solution to

$$
M A(\psi)=\mu_{0}
$$

satisfying the normalization condition

$$
\int_{X}\left(\phi-\psi_{0}\right) \mu_{0}=0
$$

Proof. Identifying $\mu_{0}$ with a probability measure on $\mathbb{P}^{n}$ we get $E_{\omega}\left(\mu_{0}\right)<\infty$, when $\omega$ is taken, fore example, as the curvature form of the Fubini-Study metric on $\mathcal{O}(1) \rightarrow \mathbb{P}^{n}$. This is shown precisely as in the proof of Lemma 3.13. The case when $\phi=\phi_{F S}$ then follows directly from the previous theorem with $u_{0}=0$. In the general case we decompose $\phi=u_{0}+\phi_{F S}$, where, by assumption $u_{0} \geq-C$ on $\mathbb{C}^{n}$. Hence, we can identify $u_{0}$ with a lsc function on $\mathbb{P}^{n}$ and apply the general form of the previous theorem.

\section{OUTLOOK AND OPEN PROBLEMS}

7.1. More general background measures and the case of compact domains in $\mathbb{C}^{n}$. A natural question is whether the volume form $d V$ appearing in Theorem 2.7 can be replaced by a more singular measure on $\mathbb{C}^{n}$. And what are the conditions that need to be imposed on $d V$ ? The following conjectural answer is motivated by the fact that the existence of a finite energy solution to the equation 2.26] only requires that $d V$ does not charge pluripolar subsets.

Conjecture 7.1. The result in Theorem 2.7 holds more generally if the assumption that $d V$ be a volume form is replaced by the assumption that $d V$ does not charge pluripolar subsets.

The conjecture does hold when $n=1$, as follows from [20, Thm 1.1, Cor 1.2]. Strictly speaking, in [20, Thm 1.1] it was assumed that $d V$ has compact support and finite energy, but the assumption on compact support may be removed by a truncation argument. Moreover, the assumption that $d V$ has finite energy was only imposed in [20, Thm 1.1] to ensure that $F_{\beta}$ is bounded from below and hence admits a minimizer $\mu_{\beta}$. But this holds more generally if $d V$ does not charge pluripolar subsets, as explained in the introduction of Section 6 , by taking $\mu_{\beta}:=M A_{\omega}\left(\varphi_{\beta}\right)$.

A natural case where the assumption in Conjecture 7.1 is satisfied is the case when

$$
d V=1_{\Omega} d \lambda
$$


for $\Omega$ a bounded open domain in $\mathbb{C}^{n}$. In this case the corresponding current $\omega^{\psi_{\beta}}$ is a weak solution to the Kähler-Einstein equation

$$
\operatorname{Ric} \omega=-\beta \omega
$$

in $\Omega$. As a complement to Conjecture rrer it seems natural to ask for conditions on $\Omega$ ensuring that $\psi_{\beta}$ be smooth?

Conjecture 7.2. Assume that $\Omega$ is an open bounded domain in $\mathbb{C}^{n}$ with smooth and strictly pseudoconvex boundary. Then, if $\beta \in\left[0, \infty\left[\right.\right.$ the solution $\psi_{\beta} \in \mathcal{L}\left(\mathbb{C}^{n}\right)$ of the equation [2.26, with $d V=1_{\Omega} d \lambda$, is smooth in $\Omega$.

Remark 7.3. This regularity problem is, in general, different than the corresponding Dirichlet problem for the equation 2.26 in $\Omega$, when $n>1$. The latter problem is known to have a smooth solution under the assumptions above [40. However, in the case when $\Omega$ is a ball it is not hard to see that the two solutions coincide (up to an additive constant) and that $\omega_{\beta}$ is the restriction to $\Omega$ of a scaled hyperbolic (Poincaré) metric when $\beta>0$.

The assumption that $\Omega$ is pseudoconvex may be superfluous. In fact, in the case when $\Omega$ is invariant under the standard action of the unit-torus Conjecture 7.2 does hold, without assuming that $\Omega$ is pseudoconvex. To see this first assume that $\beta=0$ and that $\Omega \subset \mathbb{C}^{* n}$. Denoting by $\log$ the projection from $\mathbb{C}^{* n}$ onto $\mathbb{R}^{n}$ in formula 2.37 and setting $\mu:=(\log )_{*} 1_{\Omega} d \lambda$ and $D:=\log (\Omega)$ one can check that $\psi \in \mathcal{L}\left(\mathbb{C}^{n}\right)$ solves the equation 2.26 and is in $C^{\infty}(\Omega)$ iff $\psi=\log ^{*} \phi$, where $\phi$ is strictly convex and

$$
(\nabla \phi)_{*} \mu=\nu, \quad \nu:=1_{P} d \lambda / n !
$$

where $P$ is the standard simplex in $\mathbb{R}^{n}$ (this follows from combining [22, Lemma 2.4] and [22, Lemma 3.12]). This equivalently means that the gradient map $T:=\nabla \phi$ is the optimal map transporting $\mu$ to $\nu$ in the sense of the theory of Optimal Transport (see Section 2.6.3). By the regularity results for the Optimal Transport problem in [41] it follows, using that the target $P$ is convex, that $\phi$ is in $C^{\infty}(D)$. Hence, $\psi \in C^{\infty}(\Omega)$, as desired. The case when $\Omega$ is not contained in $\mathbb{C}^{* n}$ can be handled by a change of variables argument. Moreover, the case $\beta>0$ can also be reduced to well-known regularity results in the real setting, using similar arguments.

7.2. A Green's formula for the Monge-Ampère equation $(\beta=0)$. Assume given a normalized volume form $d V$ on $\mathbb{C}^{n}$ satisfying the integrability property 3.18 . Then the unique finite energy solution $\psi \in \mathcal{E}^{1}\left(\mathbb{C}^{n}\right)$ to the complex Monge-Ampère equation

$$
M A(\psi)=d V
$$

satisfying the normalization condition 2.32, for a given weight function $\phi$, may be obtained as the double limit $\psi:=\lim _{\beta \rightarrow \infty} \lim _{k \rightarrow \infty} \psi_{\beta}^{(k)}$, where

$$
\psi_{\beta}^{(k)}:=\frac{1}{\beta} \log \frac{\int_{\left(\mathbb{C}^{n}\right)^{N_{k}-1}}\left|D^{\left(N_{k}\right)}\left(\cdot, z_{2}, \ldots z_{N_{k}}\right)\right|^{2 \beta / k}\left(e^{-\beta \phi} d V\right)^{\otimes\left(N_{k}-1\right)}}{d V}-\log Z_{N_{k}}
$$

(by combining Corollary 2.10 with Theorem2.12). Formally interchanging the two limits thus suggests the following 
Conjecture 7.4. Let $d V$ be a probability measure on $\mathbb{C}^{n}$ as above. Then there exists a sequence of constants $C_{k}$ such that

$$
\psi^{(k)}:=\frac{1}{k} \frac{\int_{\left(\mathbb{C}^{n}\right)^{N_{k}-1}} \log \left|D^{(N)}\left(\cdot, z_{2}, \ldots z_{N_{k}}\right)\right|^{2} d V^{\otimes\left(N_{k}-1\right)}}{d V}-C_{k}
$$

converges in $L_{\text {loc }}^{1}$ to a finite energy solution $\psi \in \mathcal{E}^{1}\left(\mathbb{C}^{n}\right)$ to the complex Monge-Ampère equation 7.1.

This conjecture can be seen as a non-linear generalization of the classical Green's formula for the solution of the Laplace equation in $\mathbb{C}$. Indeed, when $n=1$ formula 7.2 for $\psi^{(k)}$ coincides with the Green's formula for the solution of corresponding Laplace equation (for an appropriate choice of constants $C_{k}$ ), as follows immediately from formula 2.4.

It turns out that the validity of the conjecture above would follow from the following generalization of the Green's formula 2.5 for the classical energy in $\mathbb{C}$ :

Conjecture 7.5. Let $d V$ be a probability measure on $\mathbb{C}^{n}$ as above. Then the non-weighted pluricomplex energy $E(d V)$ can be expressed as

$$
E(d V)=\lim _{k \rightarrow \infty} \frac{1}{k N_{k}} \int_{\left(\mathbb{C}^{n}\right)^{N_{k}}} \log \left|D^{(N)}\left(z_{1}, z_{2}, \ldots z_{N_{k}}\right)\right|^{2} d V^{\otimes N_{k}}
$$

This amounts to the convergence of the mean energies 4.16 in the present setting. As discussed in [18, Section 4.3], the conjecture would follow from the conjectural LDP in the case $\beta<0$, discussed in Section 7.4.

7.3. Dynamics. We start with a general discussion, where $H^{(N)}$ should be thought of as a generic function on a space $X^{(N)}$, before coming back to the present setting, where the role of $H^{(N)}$ is played by the Hamiltonian 2.21 and $N$ is the number of points ("particles") in $\mathbb{C}^{n}$. The standard "first order" approach for finding minimizers to a given function $H^{(N)}$ on a space $X^{(N)}$ is to follow the downward gradient flow of $H^{(N)}$ :

$$
\frac{d \boldsymbol{x}(t)}{d t}=-\nabla H^{(N)}(\boldsymbol{x}(t))
$$

from appropriate initial data $\boldsymbol{x}(0)$ (assuming that $H^{(N)}$ has been endowed with a Riemannian metric). However, for numerical applications this approach is often infeasible, as the flow will typically get trapped in local minimal of $H^{(N)}$. Accordingly, a common approach used in numerical computations, is to add a noise term to the equations:

$$
d \boldsymbol{x}(t)=-\nabla H^{(N)}(\boldsymbol{x}(t)) d t+\epsilon_{N} d \boldsymbol{B}(t),
$$

where $d \boldsymbol{B}(t)$ denotes Brownian motion on $X^{(N)}$ and $\epsilon_{N}$ is a parameter measuring the strength of the noise term. From an analytic point of view the Stochastic Differential Equation (SDE) above, may be described by a linear parabolic Partial Differential Equation (PDE). Indeed, denoting by the law of the random variable $\boldsymbol{x}(t)$, its density $\rho_{t}^{(N)}$ evolves according to the forward Kolmogorov equation

$$
\frac{\partial \rho_{t}^{(N)}}{\partial t}=\frac{1}{\beta_{N}} \Delta \rho_{t}^{(N)}+\nabla \cdot\left(\rho_{t}^{(N)} \nabla H^{(N)}\right)
$$


where $\epsilon_{N}=\sqrt{\frac{2}{\beta_{N}}}$. This formulation reveals that the stationary measure of the evolution is nothing but the Gibbs measure 2.20, corresponding to the inverse temperature $\beta_{N}$ (when it is well-defined).

Remark 7.6. In the statistical mechanical literature the SDE 7.4 is called the (overdamped) Langevin equation, describing the microscopic relaxation towards equilibrium (and the PDE is called the corresponding point Fokker-Planck equation). In the statistics such evolution equations are extensively used (upon discretization) in the framework of Markov Chain Monte Carlo (MCMC) methods for sampling a given probability distribution $\rho^{(N)} d V$ on a space $X^{(N)}$. The corresponding function $H^{(N)}:=-\log \rho^{(N)}$ is then often referred to as "self-information" or "surprisal" (setting $\beta_{N}=1$ ). 2.7?

The previous discussion leads one to ask whether there is a dynamical analog of Theorem

Conjecture 7.7. Consider $\mathbb{C}^{n}$ endowed with its Euclidean metric and $\phi$ a smooth function on $\mathbb{C}^{n}$. Denote by $H_{\phi}^{\left(N_{k}\right)}$ the corresponding Hamiltonian on $\left(\mathbb{C}^{n}\right)^{N_{k}}$, defined by formula 2.21, If the initial data $x_{i}(0)$ for the $S D E 7.4$ consists of independent and identically distributed random vectors with law $\mu_{0} \in \mathcal{M}_{1}\left(\mathbb{C}^{n}\right)$ (which equivalently, means that $\mu^{(N)}(0)=\mu_{0}^{\otimes N}$ in the $P D E$ 7.5), then, at any fixed later time $t$, the corresponding empirical measures

$$
\delta_{N}(t):=N^{-1} \sum_{i=1}^{N} \delta_{x_{i}(t)}
$$

converge in law, as $N \rightarrow \infty$, to a measure $\mu_{t}=\rho_{t} d V$ on $\mathbb{C}^{n}$, which is a weak solution of the following equation on $\left.\mathbb{C}^{n} \times\right] 0, \infty[$ :

$$
\frac{\partial \rho_{t}}{\partial t}=\frac{1}{\beta} \Delta \rho_{t}-\nabla \cdot\left(\rho_{t} \nabla \psi_{t}\right)
$$

where $\psi_{t}(z) \in \mathcal{L}\left(\mathbb{C}^{n}\right)$ is a potential of $\rho_{t} d V$, i.e.

$$
M A\left(\psi_{t}\right)=\rho_{t} d V
$$

Some remarks on the conjecture:

- The form of the limiting evolution equation 7.7 is suggested by a dynamical analog of the mean field heuristics in Section 4.4, which suggest the following general evolution equation:

$$
\frac{\partial \rho_{t}}{\partial t}=\frac{1}{\beta} \Delta \rho_{t}+\nabla \cdot\left(\rho_{t} \nabla \varphi_{t}\right), \quad \varphi_{t}=d E_{\mid \mu_{t}}
$$

(which, according to Remark 3.10 yields the equation 7.7 in the present setting). In the kinetic theory literature drift-diffusion equations of the form 7.8 are usually called McKean-Vlasov equations [89, 48].

- In the terminology of Kac, if a deterministic limit $\mu_{t}$ exists, as $N \rightarrow \infty$, then propagation of chaos is said to bold.

- Formally, Theorem 2.7 corresponds to the case $t=\infty$, since the density $\rho_{\beta}$ of $\mu_{\beta}$ is a stationary solution to the evolution equation 7.7 . 
- The conjecture also naturally extends to the case of a deterministic evolution, where $\beta_{N}=\infty$ (and hence also $\beta=\infty$ ) with random initial data. Alternatively, a purely deterministic formulation is obtained by requiring that $\delta_{N}(0) \rightarrow \mu_{0}$ weakly as $N \rightarrow \infty$.

- A real analog of the conjecture is obtained when $\mathbb{C}^{n}$ is replaced by Euclidean $\mathbb{R}^{n}$ (and hence $\mu_{0}$ is supported in $\mathbb{R}^{n}$ ). Then the role of $\psi_{t}$ in equation 7.7 is played by the restriction $\psi_{t}(x)$ to $\mathbb{R}^{n} \Subset \mathbb{C}^{n}$ of the potential $\psi_{t}(z)$ of $\mu_{t}$, viewed as a probability measure on $\mathbb{C}^{n}$, supported on $\mathbb{R}^{n}$.

- Note that the conjecture makes sense also when $\phi=0$, although the corresponding stationary measure (i.e. the Gibbs measure) is not a well-defined probability measure then. Heuristically, this reflects the repulsive nature of $H_{0}^{(N)}$ : since there is no confining potential $\phi$ an initial cloud of particles will repel each other and all escape to spatial infinity as $t \rightarrow \infty$.

Unfortunately, the dynamic setting of the previous conjecture is technically considerably harder than the situation in Theorem 2.7. Indeed, it is even non-trivial to make sense of the linear PDE [7.5, sense $H_{\phi}^{(N)}$ is singular. Moreover, the existence and uniqueness of weak solutions to the non-linear equation 7.7 appears to be rather challenging.

In fact, even if $H^{(N)}$ is smooth and uniformly continuous wrt $N$, as in in the"regular case" described in Section 4.4, the corresponding result appears to be open, in general. However, assuming a uniform bound on the Hessian of $H^{(N)}$ :

$$
C^{-1} \cdot I \leq \nabla H^{(N)} \leq C \cdot I
$$

the corresponding convergence result does follow from [48] (even in the stronger sense of large deviations).

In the singular "linear" case and with the deterministic evolution 7.3 (i.e when $\beta_{N}=\infty$ ) general convergence results have very recently been obtained in [101] for repulsive "power-laws" in $\mathbb{R}^{D}$ of long-range:

$$
g(x, y)=\frac{1}{\alpha}|x-y|^{-\alpha}, \quad \alpha \in[0, D+1[,
$$

under the regularity assumption that $\mu_{t}$ admits an $L^{\infty}$-density (when $\alpha<D-1$ ). The results in [101] cover the deterministic version of Conjecture 7.7, when $n=1$ (i.e. $D=2$ ) with $\phi=0$, corresponding to the logarithmic case $\alpha=0$ (when combined with the $L^{\infty}$-estimates on $\rho_{t}$ in [86, 99]). Interestingly, this case appears in the theory of supraconductivity [47] (and superfluidity [58]), where the role of the points $x_{1}, \ldots, x_{N}$ in $\mathbb{C}$ is played by vortices (formed in a type-II supra conductor, in the realistic limit of large Ginzburg-Landau parameter).

However, when turning on a noise $\left(\beta_{N}<\infty\right)$ there seem to be very few general results in the singular linear setting, except in the case of the real line $\mathbb{R}$ (discussed in Remark 7.8 below). Recently, a strong well-posedness result was obtain for the SDE 7.4 in the logarithmic setting of $\mathbb{C}$ (when $\phi(z)=|z|^{2}$ ) with a quantitative convergence towards the corresponding Gibbs measure, as $t \rightarrow \infty$. But the conjectural convergence in the limit when $N \rightarrow \infty$ still appears to be open even in the logarithmic setting of $\mathbb{C}$.

7.3.1. Wasserstein gradient flows. A new approach for establishing the convergence as $N \rightarrow \infty$ for the empirical measures of the SDEs 7.4 was introduced in [29]. The starting point is the important observation of Otto [93, that the evolution equation 7.8 is the gradient flow of the functional $E$ on the space of all smooth probability measures on $X$, endowed with Otto's 
Riemannian metric. The latter metric coincides with Wasserstein metric, which defines a metric space structure on the space $\mathcal{P}_{2}(X)$ of all probability measures on $X$ with finite second moments. According to the general theory of gradient flows on metric spaces in [3], unique gradient flow solutions in $\mathcal{P}_{2}(X)$ exist if $E$ is assumed to be $\lambda$-convex on the metric space $\mathcal{P}_{2}\left(\mathbb{R}^{D}\right)$ Using the theory in [3] it was shown in [29] that if $H^{(N)}$ is assumed uniformly $\lambda$-convex on $\left(\mathbb{R}^{D}\right)^{N}$, i.e. if the Hessian of $H^{(N)}$ is uniformly bounded from below,

$$
\lambda \cdot I \leq \nabla H^{(N)}
$$

then the corresponding empirical measures $\delta_{N}(t)$ indeed converge in law towards the gradient flow $\mu_{t}$, as $N \rightarrow \infty$. As shown in 29] this result applies, in particular, to the "tropicalization" of the complex setting in Theorem 2.7, in the case of negative temperature (discussed in Section 2.6).

However, due to lack of convexity, new ideas are needed in the original complex setting of Conjecture 7.7. In the case of the complex plane, or more precisely when $X$ is a bounded domain in $\mathbb{C}$, gradient flow solutions $\mu_{t}$ of the corresponding logarithmic energy $E$ were constructed in [4], motivated by application to superconducitivity (when $\beta=\infty$ ). Uniqueness of $L^{\infty}$-solutions was then established in [90] (for $X$ convex). However, the convergence as $N \rightarrow \infty$ in Conjecture 7.7 remains to be established even in the case of complex plane (or a bounded domain in $\mathbb{C}$ ).

Remark 7.8. The approach in [29] also applies in the "linear" setting with a lower-law 7.9] when $D=1$. This covers the logarithmic pair interaction on the real line (corresponding to the onedimensional case of the the real analogue discussed in the fifth point after the statement of Conjecture 7.7). This case has previously been studied extensively when $\phi(x)=|x|^{2}$ in connection to Dyson's Brownian motion on the space of Hermitian random matrices.

Interestingly, the "attractive" analog of Conjecture 7.7, i.e. the case when $H^{(N)}$ is replaced by $-H^{(N)}$, which appears in the negative temperature setting discussed below, has been studied extensively in the case of the complex plane. The corresponding evolution equation 7.7. obtained by replacing $\psi_{t}$ with $-\psi_{t}$, is then called the Keller-Segal equation in chemotaxis, where the role of the points $x_{1}, . ., x_{N}$ is played by bacteria. In this setting a weaker form of the Conjecture 7.7 was recently established in [63], saying that the result holds up to passing to a subsequence of $\mu^{(N)}$.

7.4. The case of negative temperature $(\beta<0)$ and integrability indices. Assume given a convex body $P$ in $\mathbb{R}^{n}$, containing 0 in its interior and denote by $\mathcal{P}_{k P}\left(\mathbb{C}^{* n}\right)$ the corresponding space of Laurent polynomials of $\mathbb{C}^{* n}$ (introduced in Section 2.6). Fixing $\beta<0$ and a weight function $\phi \in \mathcal{L}_{P}\left(\mathbb{C}^{* n}\right)$ we denote by $\mu_{\beta}^{\left(N_{k}\right)}$ the corresponding probability measure on $\left(\mathbb{C}^{* n}\right)^{* N_{k}}$ (when it if well-defined):

$$
\mu_{\beta}^{\left(N_{k}\right)}=\frac{1}{Z_{N, \beta}}\left|D^{\left(N_{k}\right)}\left(z_{1}, \ldots, z_{N_{k}}\right)\right|_{k \phi}^{2 \beta / k} d V^{\otimes N}, d V:=e^{-\phi} d V_{\mathbb{C}^{* n}}
$$

It can be shown that $\mu_{\beta}^{\left(N_{k}\right)}$ is a well-defined probability measure, i.e. $Z_{N, \beta}<\infty$ if $\beta$ is sufficiently close to 0 . The following conjecture is motivated by its tropical analog, Theorem 2.19.

Conjecture 7.9. Let $P$ be a convex body in $\mathbb{R}^{n}$ containing 0 in its interior. 
- For $k$ sufficiently large, the corresponding Gibbs measure $\mu_{\beta}^{\left(N_{k}\right)}$ is a well-defined probability measure iff $\beta>-R_{P}$, where $\left.\left.R_{P} \in\right] 0,1\right]$ is the invariant of $P$ in formula 2.44.

- If $\beta>-R_{P}$, then the random measure $\delta_{N_{k}}$ on $\left.\left(\mathbb{C}^{* n}\right)^{N_{k}}, \mu_{\beta}^{\left(N_{k}\right)}\right)$ converges in law, as $N \rightarrow \infty$, towards $\mu_{\beta}=e^{\beta \psi_{\beta}} d V$, where $\psi_{\beta}$ is the unique solution in $\mathcal{L}_{P,+}\left(\mathbb{C}^{* n}\right)$ to the equation 2.26 on $\mathbb{C}^{* n}$.

As recalled in Section 2.6, the previous conjecture is motivated by Kähler-Einstein geometry on toric varieties. Indeed, when $\beta=-1$ a solution $\psi_{\beta}$ of the corresponding equation 2.26] is the Kähler potential of a Kähler-Einstein metric $\omega$ on $\mathbb{C}^{* n}$, which extends to the toric variety $X_{P}$, in the case when $P$ is rational polytope.

The conjecture holds when $n=1$, as follows from the stronger LDP established in 20 (applied to $X:=\mathbb{P}^{1}$ ). This is closely related to [42, 77, which consider the case of bounded domains in $\mathbb{C}$, motivated by Onsager's statistical vortex model.

Moreover, the necessary condition that $\beta>-R_{P}$ in the first point of the conjecture follows from its tropical analog, i.e. the first point in Theorem 2.19. The question whether the condition is sufficient can be formulated in terms of properties of integrability indices of psh functions (known as log canonical thresholds in the algebraic setting [79]). Briefly, if $\psi$ is in $\mathcal{L}_{P}\left(\mathbb{C}^{* m}\right)$, then its (global) integrability index $c_{\psi}$ is defined as the positive number

$$
c_{\psi}:=\sup \left\{c: \int_{\mathbb{C}^{* m}} e^{-c(\psi-\phi)} e^{-\phi} d V_{\mathbb{C}^{* n}}<\infty\right\}
$$

for any fixed $\phi \in \mathcal{L}_{P,+}\left(\mathbb{C}^{* m}\right)$. When $\psi=k^{-1} \log \left|p_{k}\right|^{2}$ for $p \in \mathcal{P}_{k P}\left(\mathbb{C}^{* m}\right)$ and the convex body $P$ is a rational polytope the number $c_{\psi}$ coincides with $k^{-1}$ times the log canonical threshold lct $\left(\mathcal{D}_{k}\right)$ of the divisor $\mathcal{D}_{k}$ in the toric variety $X_{P}$, cut-out by the zero-locus of $p_{k}$. Introducing the "tropical log canonical threshold"

$$
\operatorname{lct}\left(\mathcal{D}_{k}\right)_{\text {trop }}:=c_{\text {Log* }} \psi_{\text {trop }},
$$

where $\psi_{\text {trop }}$ is the tropicalization of $p_{k}$ and observing that

$$
\operatorname{lct}\left(\mathcal{D}_{k}\right) \leq \operatorname{lct}\left(\mathcal{D}_{k}\right)_{\text {trop }}
$$

it is natural to ask for conditions ensuring that equality holds in 7.10]. Indeed, by the first point in Theorem 2.19, the first point in Conjecture 7.9 is equivalent to proving that equality holds in 7.10, for $k$ sufficiently large, when $p_{k}=D^{\left(N_{k}\right)}$

More generally, for a given psh function $\psi \in \mathcal{L}_{P}\left(\mathbb{C}^{* n}\right)$ one can ask for conditions ensuring that $c_{\psi}=c_{\log ^{*} \psi_{\text {trop }}}$, where $\psi_{\text {trop }}$ is the convex function defined by the limit in formula 2.38, The existence of the limit, follows from [95, Theorem 2] (in the terminology of [95], the psh function $\log ^{*} \psi_{\text {trop }}=\Psi_{0}$ is called the indicator of $\psi$ at 0 ).

\section{Appendix: Comparison with the Curie-Weiss model for magnetization}

It may be illuminating to compare the present statistical mechanical setting with the classical example of the Curi-Weiss model, which provided the first microscopic explanation of the macroscopic phenomenon of spontaneous magnetization of certain materials (see [59, Section IV.4] and references therein for more details on the mathematical setup). It corresponds to the simplest possible statistical mechanical setup where the space $X$ consists of merely two points. 
8.1. The Curie-Weiss model. Let $X=\{1,-1\}$ (representing spin up/down) and consider the Hamiltonian

$$
H_{f}^{(N)}\left(x_{1}, \ldots, x_{N}\right):=-N^{-1} \frac{1}{2} \sum_{1 \leq i, j \leq N} x_{i} \cdot x_{j},
$$

representing the interaction between $N$ microscopic spins $x_{1}, \ldots x_{N}$. The subscript $f$ indicates that the system is ferromagnetic, i.e. the corresponding Gibbs measure favors ordered configuration of spins which are aligned. In particular, $H^{(N)}\left(x_{1}, \ldots, x_{N}\right)$ has precisely two minimizers $(1, \ldots, 1)$ and $(-1, \ldots-1)$ of completely aligned spins, which are exchanged under the natural $\mathbb{Z}_{2}$-action on $X^{N}$ induced by $x \mapsto-x$. This is to be contrasted with the anti-ferromagnetic setting corresponding to the Hamiltonian

$$
H_{a f}^{(N)}=-H_{f}^{(N)}
$$

which favors "disordered" configurations of spins.

In the setting of the Curie-Weiss model the "Mean Field Approximation" 4.12 is exact 4 :

$$
E_{f}^{(N)}\left(x_{1}, \ldots x_{N}\right)=E_{f}\left(\delta_{N}\right), \quad E_{f}(\mu)=-\frac{1}{2} \int x \cdot y \mu(x) \otimes \mu(y)
$$

Since $X$ has only two elements a probability measure $\mu \in \mathcal{M}_{1}(X)$ is uniquely determined by its first moment

$$
m_{\mu}:=\int_{X} x \mu(x) \in[-1,1]
$$

(representing the mean magnetic moment of the macroscopic spin state $\mu$ ). Accordingly, the empirical measure $\delta_{N}$ is uniquely determined by the random variable

$$
m_{N}:=m_{\delta_{N}}:=N^{-1} \sum_{i=1}^{N} x_{i}
$$

(representing the mean spin of the microscopic state), taking values in $[-1,1]$. Expressing $E$ in terms of $m$ gives, by separation of variables,

$$
E_{f}(\mu)=-\frac{1}{2} m^{2}
$$

Hence, writing $I_{\beta}(m):=F_{\beta}(\mu)$ the corresponding rate functional is given by

$$
F_{\beta}(m):=-\frac{1}{2} m^{2}+\beta^{-1}\left(\frac{m+1}{2} \log \left(\frac{m+1}{2}\right)+\frac{1-m}{2} \log \left(\frac{1-m}{2}\right)\right)
$$

for $m \in[-1,1]$. The corresponding critical point equation yields the following classical mean field equation for the magnetization $m$ :

$$
m=\tanh (\beta m)
$$

\footnotetext{
${ }^{4}$ However, in the physics literature the Hamiltonian $H^{(N)}$ is itself viewed as a mean field approximation of more complicated spin models, such as the Ising model, on a lattice $\mathbb{Z}^{D}$ (which improves as the dimension $D$ is taken large [30]).
} 
(which is consistent, as it much with equation 4.17, using that $\psi_{\mu}(x)=-x \cdot m_{\mu}$ ). Inspection of the graph of $\tanh s$ reveals that the $m=0$ is the unique solution when $\beta \in[0,1]$ (and thus the unique minimizer of $F_{\beta}$ ). However, when

$$
\beta>\beta_{c}:=1
$$

(the "critical inverse temperature") there are two additional solutions $m_{ \pm}(\beta)$ which are exchanged by the $\mathbb{Z}_{2}$-symmetry (i.e. $m_{-}(\beta)=-m_{+}(\beta)$ ). The solutions $m_{ \pm}(\beta)$ minimize of $F_{\beta}$, while $m=0$ is now a local maximize. Moreover, $m_{ \pm}(\beta) \rightarrow \pm 1$ when $\beta \rightarrow \infty$, coinciding with the two minimizers of $E_{f}(m)$ (representing a macroscopic state of all spins up, respectively down).

The LDP for the laws of $m_{N}$ thus implies that

$$
\beta<\beta_{c} \Longrightarrow m_{N} \rightarrow 0, N \rightarrow \infty
$$

in law (which means that the system is not magnetized for sufficiently large temperatures). However, when $\beta>\beta_{c}$ it follows, from the LDP combined with the $\mathbb{Z}_{2}-$ symmetry of $H_{f}^{(N)}$ that the laws of $m_{N}$ converge to $\delta_{\mu_{+}(\beta)} / 2+\delta_{-\mu_{+}(\beta)} / 2$. Hence, $m_{N}$ does not admit a deterministic limit, even though the expectations of $m_{N}$ vanish identically for any $N$ (i.e. the law of large numbers fails in this case). This is the standard example of a (first order) phase transition, appearing at the critical inverse temperature $\beta=\beta_{c}$.

8.1.1. Spontaneous symmetry breaking. Determinism is restored in the presence of an exterior magnetic field, which leads to the phenomenon of spontaneous symmetry breaking. Indeed, turning on an exterior magnetic field $h \in \mathbb{R}$ amounts to adding a linear exterior potential $\phi_{h}(x):=-x \cdot h$ to the Hamiltonian $H_{f}^{(N)}$. Then the corresponding rate functional $I_{\beta, h}(m)$ has a unique minimizer $m(\beta, h)$ for any $h \neq 0$. Moreover,

$$
\lim _{h \rightarrow 0^{ \pm}} m(\beta, h)=m_{ \pm}(\beta)
$$

This means that the random variables $m_{N}$ on the corresponding probability spaces $\left(X^{N}, \mu_{\beta, h}^{(N)}\right)$ do have a deterministic limit:

$$
\beta>\beta_{c} \Longrightarrow m_{N} \rightarrow m_{ \pm}(\beta, h), \quad N \rightarrow \infty
$$

in law, which is viewed as a microscopic explanation of the phenomenon of spontaneous magnetization, observed in certain materials (such as iron).

8.2. Comparison with the present complex setting. To make a comparison with the present setting of interpolation nodes in $\mathbb{C}^{n}$ it is more convenient to start with the antiferromagnetic Hamiltonian setting described by the Hamiltonian

$$
H^{(N)}:=N^{-1} \frac{1}{2} \sum_{1 \leq i, j \leq N} x_{i} \cdot x_{j}
$$

Loosely speaking, the Hamiltonian $H^{(N)}$ is "repulsive", since the ferromagnetic Hamiltonian $-H^{(N)}$ is "attractive" in the sense that $H^{(N)}\left(x_{1}, \ldots, x_{N}\right)$ is minimal precisely when all $x_{i}$ coincide. In this setting the critical inverse temperature $\beta_{c}$, where magnetization appears, is negative, $\beta_{c}=-1$. The critical $\beta_{c}$ may abstractly be defined as

$$
\beta_{c}:=\inf \left\{\beta: F_{\beta} \text { has a unique minimizer }\right\}
$$


Thus $\beta_{c}$ is analogous to the critical negative inverse temperature $\beta_{c}$ for the repulsive determinantal Hamiltonian $H^{(N)}$ (formula 2.21) which appears in the present setting of $\mathbb{C}^{n}$, discussed in Section 2.6. In the latter setting the role of symmetry group $\mathbb{Z}^{2}$ is played by the group $\mathbb{C}^{* n}$. This analogy becomes even more striking in the tropical setting in $\mathbb{R}^{n}$, where the symmetry group is te additive group $\mathbb{R}^{n}$ and where it can be shown that the corresponding attractive Hamiltonian $H_{\text {trop }}^{(N)}\left(x_{1}, \ldots, x_{N}\right)$ is regular and minimal precisely when all points coincide, $x_{1}=\ldots .=x_{N}$.

Remark 8.1. Negative temperature states were first observed in nuclear spins in the famous experiment 72 (and then in 72, for an antiferromagnetic model for silver, whose mean field Hamiltonian coincides with 8.1). However, the existence of negative temperature states was originally proposed by Onsager for motional degrees of freedoms in the vortex model (see the historical account in 61]). Incidentally, Onsager's Hamiltonian coincides with the present one (formula 2.21) in the non-weighted setting of $\mathbb{C}$ (i.e. when $n=1$ ). Note that the term "negative temperature" is somewhat misleading, though, as such states are hotter then infinite temperature states. Indeed, the inverse temperature $\beta$ interpolates continuous between positive and negative temperature states, passing through the infinite temperature state $\beta=0$ (see [1] for a recent theoretical discussion of negative temperature states in physics).

\section{REFERENCES}

[1] Eitan Abraham and Oliver Penrose: Physics of negative absolute temperatures. Phys. Rev. E 95 (2017)

[2] G Akemann, SS Byun:The high temperature crossover for general 2D Coulomb gases. arXiv:1808.00319

[3] Ambrosio, L; Gigli, N; Savaree,G: Gradient flows in metric spaces and in the space of probability measures Lectures in Mathematics ETH Zurich. Birkhauser Verlag, Basel, 2005.

[4] Ambrosio, L; Serfaty, S: A gradient flow approach to an evolution problem arising in superconductivity. Commun. on Pure and Applied Math. Vol 61, Issue11 (2008).

[5] Aubin, T: Equations du type Monge-Amp'ere sur les vari 'et 'es K“" ahl 'eriennes com- pactes", Bull. Sc. Math. 102 (1978).

[6] Aubin, T. R'eduction de cas positif de l' 'equation de Monge-Amp'ere s ur les vari 'et 'es k"ahl'eriennes compactes 'a la d'emonstration d'une in'eg alit'e. J. Funct. Anal. 57 , 2 (1984), 143-153

[7] Turgay Bayraktar, Thomas Bloom, Norman Levenberg: Pluripotential Theory and Convex Bodies. Sbornik Mathematics 209 (2018) no.3 67-101

[8] E. Bedford, A. Taylor. : A new capacity for plurisubharmonic functions. Acta Math., 149:1--40, 1982

[9] E. Bedford, A. Taylor. The complex equilibrium measure of a symmetric convex set in $\mathrm{R}^{\wedge} \mathrm{n}$. Trans. Amer. Math. Soc. 294 (1986), pp. 705-717

[10] Boucksom, S; Essidieux,P: Guedj,V; Zeriahi: Monge-Ampere equations in big cohomology classes. Acta Math. 205 (2010), no. 2, 199-262.

[11] Berman, R.J: Bergman kernels and equilibrium measures for line bundles over projective manifolds. The American J. of Math., Vol 131, Nr. 5, 2009

[12] Berman, R.J: Bergman kernels for weighted polynomials and weighted equilibrium measures of $\mathrm{C}^{\wedge} \mathrm{n} .19$ pages. Indiana Univ. Math. Journal, Volume 58, issue 4, 2009

[13] Berman, R.J: Determinantal point processes and fermions on complex manifolds: Large deviations and Bosonization. Comm. in Math. Phys. Volume 327, Issue 1 (2014), Page 1-47

[14] Berman, R.J: Kahler-Einstein metrics emerging from free fermions and statistical mechanics. 22 pages, J. of High Energy Phys. (JHEP), Volume 2011, Issue 10 (2011)

[15] Berman, R.J: A thermodynamical formalism for Monge-Ampere equations, Moser-Trudinger inequalities and Kahler-Einstein metrics. Advances in Math. 1254. Volume: 248. 2013

[16] Berman, R.J: K-polystability of Q-Fano varieties admitting Kahler-Einstein metrics. Inventiones Math. March 2016, Volume 203, Issue 3, pp 973-1025

[17] Berman, R.J: Statistical mechanics of permanents, real-Monge-Ampere equations and optimal transport. arXiv:1302.4045 
[18] Berman, R.J: Large deviations for Gibbs measures with singular Hamiltonians and emergence of KählerEinstein metrics. Communications in Math. Phys. (to appear).

[19] Berman, R.J: Kähler-Einstein metrics, canonical random point processes and birational geometry. AMS Proceedings of the 2015 Summer Research Institute on Algebraic Geometry (to appear)

[20] Berman, R.J: On large deviations for Gibbs measures, mean energy and Gamma convergence. Constructive Approximation. Volume 48, Issue 1 (2018) pp 3-30

[21] Berman, R.J: From Monge-Ampere equations to envelopes and geodesic rays in the zero temperature limit. arXiv:1307.3008. Math. Z (to appear).

[22] Berman, R.J: Convergence rates for discretized Monge-Ampère equations and quantitative stability of Optimal Transport. https://arxiv.org/abs/1803.00785

[23] Berman, R.J: A probabilistic analog of the Yau-Tian-Donaldson conjecture on toric varieties and tropicalization. In preparation.

[24] Berman, R.J: Potential theory, the Coulomb gas and phase transitions. In preparation.

[25] R.J.Berman;B. Berndtsson: Real Monge-Ampere equations and Kahler-Ricci solitons on toric log Fano varieties. Les Annales de la Faculté des Sciences de Toulouse (2013) vol. $22 \mathrm{n}^{\circ} 4$

[26] Berman, R.J.; Boucksom, S: Growth of balls of holomorphic sections and energy at equilibrium. Invent. Math. Vol. 181, Issue 2 (2010), p. 337

[27] Berman, R.J.; Boucksom, S; Witt Nyström, D: Fekete points and convergence towards equilibrium measures on complex manifolds. Acta Math. Vol. 207, Issue 1 (2011), 1-27

[28] Berman, R.J; Boucksom, S; Guedj,V; Zeriahi: A variational approach to complex Monge-Ampere equations. Publications math. de l'IHÉS (2012): 1-67, November 14, 2012

[29] R.J. Berman, M. Onnheim: Propagation of chaos, Wasserstein gradient flows and toric Kahler-Einstein metrics. Anal. PDE Volume 11, Number 6 (2018), pp 1343-1380

[30] Biskup, M; Chayes, L: Rigorous analysis of discontinuous phase transitions via mean-field bounds. Comm. Math. Phys. 238 (2003), no. 1-2, 53-93.

[31] François Bolley, Djalil Chafai, Joaquín Fontbona: Dynamics of a planar Coulomb gas. http://arxiv.org/abs/1706.08776v3

[32] T. Bloom and N. Levenberg, Asymptotics for Christoffel functions of planar measures, Journal d'Analyse Math. , 106 (2008), 353-371.

[33] T. Bloom and N. Levenberg, Pluripotential energy and large deviation, Indiana Univ. Math. J. , 62 (2013), no. 2, 523-5

[34] Bloom, T; Levenberg, N;Piazzon, F; Wielonsky, F: Bernstein-Markov: a survey. Dolomites Research Notes on Approximation. Special Issue for the "10 years of the Padua points", Volume 8 - 2015 · Pages 75-91

[35] T. Bloom and B. Shiffman, Zeros of random polynomials on Cm, Math. Res. Lett. 14 (2007), 469-479.

[36] Len Bos, Stefano De Marchi, Alvise Sommariva, Marco Vianello, Computing multivariate Fekete and Leja points by numerical linear algebra, SIAM J. Numer. Anal. 48 (5) (2010) 1984-1999

[37] J. Boyd: Chebyshev and Fourier Spectral Methods, C.A. Brebbia and S.A. Orszag, eds., Lecture Notes in Engrg. 49, Springer-Verlag, New York, 1989

[38] Braides, A: Gamma-convergence for beginners. Oxford University Press, 2002.

[39] J. M. Cailloi; D. Levesque; i j. j. Weis and J. P. Hansen: A Monte Carlo study of the classical twodimensional one-component plasma

[40] L. Caffarelli, J. J. Kohn, L. Nirenberg;J. Spruck: The Dirichlet problem for nonlinear second-order elliptic equations. II. Complex Monge-Amp'ere, and uniformly elliptic equations, Comm. Pure Appl. Math. 38 (1985), no. 2, 209-252

[41] Caffarelli, L. A: The regularity of mappings with a convex potential. J. Amer. Math. Soc. 5 (1992), no. 1, 99-104

[42] Caglioti.E; Lions, P-L; Marchioro.C; Pulvirenti.M: A special class of stationary flows for two-dimensional Euler equations: a statistical mechanics description. Communications in Mathematical Physics (1992) Volume 143, Number 3, 501-525

[43] J. P. Calvi and N. Levenberg, Uniform approximation by discrete least squares polynomials , J. Approx. Theory, 152 (2008), pp. 82-100.

[44] T. Carroll, J. Marzo, X. Massaneda, J. Ortega-Cerd'a, Equidistribution and $\beta$-ensembles. Preprint (2015). arXiv:1509.06725 
[45] Chafaï, D; Gozlan, N; Zitt, P-A: First-order global asymptotics for confined particles with singular pair repulsion. Ann. Appl. Probab. 24 (2014), no. 6, 2371-2413.

[46] Chu J., Zhou B: Optimal regularity of plurisubharmonic envelopes on compact Hermitian manifolds. preprint arXiv:1702.05230

[47] S. J. Chapman, J. Rubinstein, and M. Schatzman. A mean field model of superconducting vortices. European J. Appl. Math. , 7(2):97-111, 1996.

[48] DA Dawson, J Gärtner: Large deviations from the McKean-Vlasov limit for weakly interacting diffusions. Stochastics Volume 20, Issue 4, 1987

[49] Demailly, J. P: Complex analytic and differential geometry. Book available at https://www-fourier.ujfgrenoble.fr/ $\sim$ demailly/manuscripts/agbook.pdf

[50] Darvas, T; Rubinstein, YA: Kiselman's principle, the Dirichlet problem for the Monge-Ampere equation, and rooftop obstacle problems. arXiv:1405.6548, 2014

[51] T-C. Dinh, X. Ma, V.-A. Nguyen, Equidistribution speed for Fekete points associated with an ample line bundle. Preprint (2015). arXiv:1505.0805

[52] T-C. Dinh; V.-A. Nguyen: Large deviations for some beta ensembles. arXiv:1603.03643

[53] Dembo, A; Zeitouni O: Large deviation techniques and applications. Corrected reprint of the second (1998) edition. Stochastic Modelling and Applied Probability, 38. Springer-Verlag, Berlin, 2010. xvi +396 pp.

[54] Donaldson, S: Stability of algebraic varieties and Kahler geometry. Algebraic geometry: Salt Lake City 2015, PT 1, Vol: 97, Pages: 199-221,

[55] Dumitriu, I., Edelman, A.: Matrix models for beta ensembles. J. Math. Phys. 43 (11), 5830-5847 (2002)

[56] Dupuis, P; Laschos, V; Ramanan, K: Large deviations for empirical measures generated by Gibbs measures with singular energy functionals. arXiv:1511.06928

[57] Trinh Khanh Duy, Tomoyuki Shirai: The mean spectral measures of random Jacobi matrices related to Gaussian beta ensembles. Electron. Commun. Probab. 20 (2015), no. 68, 1--13

[58] E, W: Dynamics of vortex-liquids in Ginzburg-Landau theories with applications to super- conductivity . Phys. Rev. B 50 (1994), no. 3, 1126-1135

[59] Ellis, R. S: Entropy, Large Deviations, and Statistical Mechanics. Springer-Verlag (2012).

[60] Ellis, R. S.; Haven, K; Turkington, B: Large deviation principles and complete equivalence and nonequivalence results for pure and mixed ensembles. J. Statist. Phys. 101 (2000), no. 5-6, 999-1064

[61] Eyink, G.L; Sreenivasan, K.R: Onsager and the theory of hydrodynamic turbulence. Reviews of Modern Physics. Vol. 78 (2006), no. 1, 87-135

[62] Forrester, Peter J.; Warnaar, S. Ole: The importance of the Selberg integral. Bull. Amer. Math. Soc. (N.S.) 45 (2008), no. 4, 489-534.

[63] Fournier, N; Benjamin Jourdain, B: Stochastic particle approximation of the Keller-Segel equation and two-dimensional generalization of Bessel processes. http://arxiv.org/pdf/1507.01087v1.pdf

[64] Eyssidieux, P; Guedj, V; Zeriahi, A: Viscosity solutions to degenerate complex Monge-Ampère equations. Comm. Pure Appl. Math. 64 (2011), no. 8, 1059-1094.

[65] Eyssidieux, P; Guedj, V; Zeriahi, A: Erratum to Viscosity solutions to complex Monge-Amp $\backslash$ ere equations. arXiv:1610.03087

[66] Fejer, L., Bestimmung derjenigen Abszissen eines Intervalles f ur welche die Quadratsumme der Grundfunktionen der Lagrangeschen Interpolation im Intervalle [-1,1] ein m oglichst kleines Maximum besitzt, Ann. Scuola Norm. Sup. Pisa Sci. Fis. Mt. Ser. II , 1 , 263\{276, 1932

[67] García Zelada, D: A large deviation principle for empirical measures on Polish spaces: Application to singular Gibbs measures on manifolds. arXiv:1703.02680

[68] Guedj,V; Zeriahi, A: Intrinsic capacities on compact Kähler manifolds. J. Geom. Anal. 15 (2005), no. 4, 607-639.

[69] Guedj,V; Zeriahi, A: Degenerate Complex Monge-Ampère Equations. EMS Tracts in Mathematics Vol. 26

[70] Guedj,V; Lu, C.H: Zeriahi: Plurisubharmonic envelopes and supersolutions. Arxiv: 1703.05254

[71] G“ otz,M.,Maymeskul,V.V.\&Saff,E.B. Asymptotic distribution of nodes for near-optimal polynomial interpolation on certain curves in $\mathrm{R}^{\wedge} 2$. Constr. Approx. , 18 (2002), 255-283

[72] Hakonen PJ, Nummila KK, Vuorinen RT, Lounasmaa OV: Observation of nuclear ferromagnetic ordering in silver at negative nanokelvin temperatures. Phys Rev Lett. 1992 Jan 20;68(3):365-368 
[73] Hough, J. B.; Krishnapur, M.; Peres, Y.l; Virág, B: Determinantal processes and independence. Probab. Surv. 3 (2006), 206-229

[74] Hultgren, J: Permanental point processes on real tori, theta functions and Monge-Ampere equations. arXiv:1604.05645 (To appear in Annales de la faculté des sciences de Toulouse).

[75] Itenberg, I.; Mikhalkin, G. Geometry in the tropical limit. Math. Semesterber. 59 (2012), no. 1, 57-73

[76] Jeffres, T; Mazzeo, R; Rubinstein, Y.A:; Kähler-Einstein metrics with edge singularities. Annals of Math. Volume 183 (2016), Issue 1, Pages 95-176

[77] Kiessling M.K.H.: Statistical mechanics of classical particles with logarithmic interactions, Comm. Pure Appl. Math. 46 (1993), 27-56.

[78] Klevtsov, S: Geometry and large N limits in Laughlin states. arXiv:1608.02928

[79] Kollar, J: Singularities of pairs. Algebraic geometry - Santa Cruz 1995, 221-287.

[80] Krishnapur, M: From random matrices to random analytic functions. Ann. Probab. Volume 37, Number 1 (2009), 314-346.

[81] N. Lev, J. Ortega-Cerd'a, Equidistribution estimates for Fekete points on complex manifolds, J. Eur. Math. Soc. (JEMS) 18 (2016), no. 2, 425-464

[82] Levenberg, N. , Approximation in C N . Surv. Approx. Theory , 2 (2006), 92-140.

[83] Li, P; Schoen, R: Lp and mean value properties of subharmonic functions on Riemannian manifolds. Acta Math. 153 (1984), no. 3-4, 279-301.

[84] Lim, C; Nebus, J: Vorticity, statistical mechanics, and Monte Carlo simulation. Springer, 2007

[85] CC Lim, J Nebus, SM Assad: A Monte Carlo algorithm for free and coaxial ring extremal states of the vortex N-body problem on a sphere. Physica A: Statistical Mechanics and its Applications Volume 328, Issues 1-2, 1 October 2003, Pages 53-96

[86] F. H. Lin, P. Zhang, On the hydrodynamic limit of Ginzbur g-Landau vortices, Disc. Cont. Dyn. Systems 6 (2000), 121-142.

[87] Jun S. Liu: Monte Carlo Strategies in Scientific Computing. Springer 2004.

[88] Chinh H. Lu, Van-Dong Nguyen: Degenerate complex Hessian equations on compact Kähler manifolds. arXiv:1402.5147

[89] HP McKean Jr: Propagation of chaos for a class of non-linear parabolic equations. Stochastic Differential Equations (Lecture series in differential equations, session 7, Catholic University, 1967) 01/1967; 2.

[90] E. Mainini, A global uniqueness result for an evolution problem arising in superconductivity, Boll. Unione Mat. Ital. (9) II (2) (2009) 509-528.

[91] M.L. Mehta, Random Matrices ,3rd ed., Pure and Applied Mathematics, Vol. 142, Else- vier/Academic Press, Amsterdam, 2004.

[92] Messer, J; Spohn, H: Statistical mechanics of the isothermal Lane-Emden equation. J. Statist. Phys. 29 (1982), no. 3, 561-578

[93] Felix Otto. The geometry of dissipative evolution equations: the porous medium equation. Comm. Partial Differential Equations , 26(1-2):101-174, 2001

[94] Purcell, E. M.; Pound, R. V. A Nuclear Spin System at Negative Temperature. Physical Review. 81 (2): 279-280 (1951).

[95] Rashkovski, A: Indicators for plurisubharmonic functions of logarithmic growth, Indiana Univ. Math. J. 50 (2001), no. 3, 1433-1446.

[96] M. Reimer: Constructive Theory of Multivariate Functions , BI Wissenschaftsverlag, Mannheim, Wien, Z* urich, 1990.

[97] Rubinstein, Y.A: Some discretizations of geometric evolution equations and the Ricci iteration on the space of K“ahler metrics, Adv. Math. 218 (2008), 1526-1565.

[98] Saff, E. B. \& Totik, V. , Logarithmic Potentials with External Fields . Grundlehren der Mathematischen Wissenschaften, 316. Springer, Berlin-Heidelberg, 1997

[99] S. Serfaty and J. L. Vázquez. A mean field equation as limi t of nonlinear diffusions with fractional Laplacian operators. Calc. Var. Partial Differential Equations , 49(3-4):1091-1120, 2014

[100] Serfaty, S: Coulomb gases and Ginzburg-Landau vortices. Zurich Lectures in Advanced Mathematics. European Mathematical Society (EMS), Zürich, 2015. viii +157 pp

[101] S. Serfaty: Mean Field Limit for Coulomb Flows. arXiv:1803.08345

[102] . J. Siciak, Extremal plurisubharmonic functions in $\mathrm{C}^{\wedge} \mathrm{n}$, Proc. 1st Finnish-Polish Summer School in Complex Analysis, 1977, pp. 115-152. 
[103] J. Siciak: Extremal plurisubharmonic functions in $\mathrm{C}^{\wedge}$ n. Ann. Pol. Math., 39:175-211, 1981

[104] J. Siciak: Extremal plurisubharmonic functions and capacities in $\mathrm{C}^{\wedge} n$. Sophia Kokyusoku in Mathematics, 14, 1982

[105] Sloan, Ian H.;Womersley, Robert S: Extremal systems of points and numerical integration on the sphere. Adv. Comput. Math. 21 (2004), no. 1-2, 107-125.

[106] Smale S. 2000 Mathematical problems for the next century, Mathematics: Frontiers and Per- spectives 271-294, AMS Providence RI

[107] Herbert Stahl, Johannes Steel, V. Totik: General Orthogonal Polynomials. Encyclopedia of Math. Cambridge Univ. Press (1992).

[108] Touchette, H: The large deviation approach to statistical mechanics. Phys. Rep. 478 (2009), no. 1-3, $1-69$.

[109] . V. Zaharjuta, Orthogonal polynomials and the Bernstein-Walsh theorem for analytic functions of several comples variables, Ann. Polon. Math. 33 (1976), 137-148

[110] Yau, S-T: On the Ricci curvature of a compact K“ ahler manifold and the complex Monge- Amp'ere equation", Comm. Pure Appl. Math. 31 (1978)

Robert J. Berman, Mathematical Sciences, Chalmers University of Technology and the University of Gothenburg, SE-412 96 Göteborg, Sweden

E-mail address: robertb@chalmers.se 